International Journal of Modern Physics B

Vol. 32, No. 8 (2018) 1850090 (73 pages)

(C) The Author(s)

DOI: $10.1142 / \mathrm{S} 021797921850090 \mathrm{X}$

\title{
Knot physics on entangled vortex-membranes: Classification, dynamics and effective theory
}

\author{
Su-Peng Kou \\ Center for Advanced Quantum Studies, \\ Department of Physics, Beijing Normal University, \\ Beijing 100875, P. R. China \\ spkou@bnu.edu.cn
}

Received 15 October 2017

Accepted 15 November 2017

Published 6 February 2018

\begin{abstract}
In this paper, knot physics on entangled vortex-membranes are studied including classification, knot dynamics and effective theory. The physics objects in this paper are entangled vortex-membranes that are called composite knot-crystals. Under projection, a composite knot-crystal is reduced to coupled zero-lattices. In the continuum limit, the effective theories of coupled zero-lattices become quantum field theories. After considering the topological interplay between knots and different types of zero-lattices, gauge interactions emerge. Based on a particular composite knot-crystal (we call it a standard knot-crystal), the derived effective model becomes the Standard Model. As a result, the knot physics may provide an alternative interpretation on quantum field theory.
\end{abstract}

Keywords: Vortex; Kelvin waves; knot; quantum field theory.

PACS numbers: 67.25.dk, 47.32.C-, 47.37.+q

\section{Introduction}

A vortex (point-vortex, vortex-line, vortex-membrane) is among the most important and the most studied objects of fluid mechanics that consists of the rotating motion of fluid around a common center (a point, a line or a membrane). In threedimensional (3D) superfluid (SF), it has been known that a vortex-line is subject to wavy distortions called Kelvin waves. ${ }^{1,2}$ Because Kelvin waves are relevant to Kolmogorov-like turbulence, ${ }^{3,4}$ a variety of approaches have been used to study this phenomenon. For two entangled vortex-rings, there may exist the leapfrogging motion in classical fluids according to the works of Helmholtz and Kelvin. ${ }^{-10}$

This is an Open Access article published by World Scientific Publishing Company. It is distributed under the terms of the Creative Commons Attribution 4.0 (CC-BY) License. Further distribution of this work is permitted, provided the original work is properly cited. 
Kelvin came to the idea that classical atoms were knots of swirling vortices in the luminiferous aether. Chemical elements correspond to knots and links. The study of knotted vortex-lines and their dynamics has attracted scientists from diverse settings, including classical fluid dynamics and SF dynamics. $.11,12$

In Ref. 13, the Kelvin wave and knot dynamics on 3D vortex-membranes in five-dimensional (5D) fluid were studied. A new theory, knot physics, is developed to characterize the entanglement evolution of $3 \mathrm{D}$ leapfrogging vortex-membranes. Owing to the conservation conditions of the volume of the knot in 5D space, the shape and the volume of knot are never changed and the knot can only split and knot-pieces evolute following the equation of motion of Schrödinger equation. 3D quantum Dirac model is derived to describe the entanglement evolution of the entangled vortex-membranes: the elementary excitations are knots with a projected zero; the physics quantity to describe local deformation is knot density or the density of zeros between two projected vortex-membranes; the Biot-Savart equation for Kelvin waves becomes Schrödinger equation for probability waves of knots; the angular frequency for leapfrogging motion turns into the mass of knots, etc. The knot physics may give an alternative interpretation on quantum mechanics.

In this paper, we will study the Kelvin wave and knot dynamics on complex entangled vortex-membranes - a composite knot-crystal. By projecting entangled vortex-membranes into several coupled zero-lattices ( $T$-zero-lattices and $W$-zerolattices), the information of the system becomes the coupled zero-lattices with internal degrees of freedom. After considering the topological interplay between knots and zero-lattices, different kinds of gauge interactions emerge. In particular, they are the 3D quantum gauge field theories that characterize the knot dynamics of the composite knot-crystal. The knot physics may give a complete interpretation on quantum chromodynamics (QCD) and quantum electrodynamics (QED): the collective motions of composite knot-crystal are described by quantum fluctuations of zero-lattices: fermionic elementary particles are knots that are topological defects of zero-lattices with internal twistings; U(1) gauge field (Maxwell field) are phase fluctuations of internal twistings of internal T-type zero-lattice; SU(N) Yang-Mills field are number fluctuations of internal twistings of $T$-type zero-lattice. Two composite knots interact by exchanging fluctuations of the internal twistings. Based on a particular composite knot-crystal with $(\mathcal{N}=4, \mathcal{M}=3)$ (we call it a standard knot-crystal), the derived effective model is just the (one-flavor) Standard Model.

Figure 1 is the key theoretical structure of this paper. In this figure, we take a one-dimensional (1D) knot-crystal with $(\mathcal{N}=2, \mathcal{M}=1)$ as an example. Our starting point is composite knot-crystal. Under projection, a composite knot-crystal turns into coupled zero-lattices. In the continuum limit, the effective theories of coupled zero-lattices become quantum (gauge) field theories.

The paper is organized as follows. In Sec. 2, we review Biot-Savart mechanics. In Sec. 3, we give the mathematical definition of composite knot-crystal and show the classification of composite knot-crystals. In Sec. 4, we introduce the zero-lattices by projecting knot-crystals and show a topological constraint — twist-writhe locking 


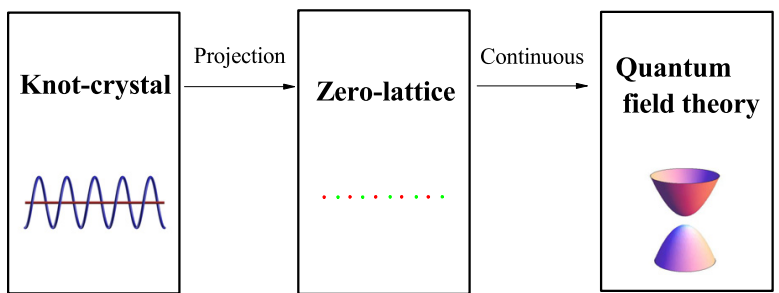

Fig. 1. (Color online) The key theoretical point of the paper. In this figure, we take a $1 \mathrm{D}$ knotcrystal with $(\mathcal{N}=2, \mathcal{M}=1)$ as an example.

condition for a composite knot-crystal. In Sec. 5, we obtain the effective theory for knot on a 1-level knot-crystal with $(\mathcal{N}=1, \mathcal{M}=1)$ and the knots are described by Weyl equation. In Sec. 6, we show the dynamics of 1-level knot-crystal with $(\mathcal{N}=2$, $\mathcal{M}=1$ ) and the knots are described by Dirac equation. In Sec. 7 , we obtain the effective theory of knots on a 2-level knot-crystal with $(\mathcal{N}=2, \mathcal{M}=2)$ and the collective motions of composite knot-crystal are described by a chiral $\operatorname{SU}_{\text {weak }}(2)$ gauge theory with Weyl fermions and Dirac fermions. In Sec. 8, we obtain the effect theory of knots on a 2-level knot-crystal with $(\mathcal{N}=4, \mathcal{M}=2)$ and the collective motions of composite knot-crystal are described by $\mathrm{SU}_{\text {strong }}(n) \times \mathrm{U}_{\mathrm{em}}(1)$ gauge field theory. In this section, the $\mathrm{SU}_{\text {strong }}(n) \times \mathrm{U}_{\mathrm{em}}(1)$ gauge fields can be viewed as fluctuations of the internal twistings. The knots correspond to quarks and electrons. In Sec. 9, based on a 3-level composite knot-crystal with $(\mathcal{N}=4$, $\mathcal{M}=3$ ) (we call it a standard knot-crystal), the derived effective model becomes the (one-flavor) Standard Model with $\mathrm{SU}_{\text {strong }}(n) \times \mathrm{U}_{\mathrm{em}}(1) \times \mathrm{SU}_{\text {weak }}(2)$ gauge fields. The knots correspond to quarks, electrons and neutrinos. Finally, the conclusions are drawn in Sec. 10.

\section{Review on Biot-Savart Mechanics}

In Ref. 13, to characterize the entanglement evolution of vortex-membranes, a new theory, knot physics, was developed from Biot-Savart mechanics. In this paper, firstly, we review the key points of the Biot-Savart mechanics.

The $3 \mathrm{D}$ vortex-membrane is defined by a given singular vorticity $\Omega=\kappa \delta_{P}$ in the $5 \mathrm{D}$ inviscid incompressible fluid $(\nabla \cdot \mathbf{v} \equiv 0)$, the singular $\delta$-type vorticity denotes the submanifold $P$ in $5 \mathrm{D}$ space and $\kappa$ is the constant circulation strength. For $5 \mathrm{D}$ case, we have 3D vortex-membranes with Marsden-Weinstein (MW) symplectic structure. 14

The generalized Biot-Savart equation for a 3D vortex-piece under local induction approximation (LIA) can be described by Hamiltonian formula:

$$
\dot{\xi}=\frac{\partial \mathrm{H}_{\text {volume }}(P)}{\partial \eta}, \quad \dot{\eta}=-\frac{\partial \mathrm{H}_{\text {volume }}(P)}{\partial \xi},
$$

where the Hamiltonian on the vortex-membranes is just three-volume

$$
\mathrm{H}_{\text {volume }}(P)=(\kappa \alpha \ln \epsilon) \cdot \operatorname{volume}(P)
$$


with volume $(P)=\int_{P} d V_{P}$ and $\alpha=\frac{\Gamma\left(\frac{5}{2}\right)}{6 \pi^{\frac{5}{2}}}$. Here, $\epsilon$ is defined by $\epsilon=\frac{\ell}{a_{0}}$, where $\ell$ is the length of the order of the curvature radius (or inter-vortex distance when the considered infinitesimal vortex is a part of a vortex tangle) and $a_{0}$ denotes the infinitesimal vortex radius which is much smaller than any other characteristic size in the system. In complex description, $\mathrm{z}=\xi+i \eta$, above equation can also be written $\operatorname{as}^{14} i \frac{d \mathrm{z}}{d t}=\frac{\delta \mathrm{H}_{\text {volume }}(P)}{\delta \mathrm{z}^{*}}$.

For Kelvin waves on a 3D helical vortex-membrane, the plane wave is described by a complex field, $\mathrm{z}(\vec{x}, t)=r_{0} e^{ \pm i \vec{k} \cdot \vec{x}-i \omega t+i \phi_{0}}$ where $\vec{k}$ is the winding wave vector along a direction on $3 \mathrm{D}$ vortex-membrane with $|\vec{k}|=\frac{\pi}{a}$ and $a$ is a fixed length that denotes the half pitch of the windings. The (Lamb impulse) momentum and the (Lamb impulse) angular momentum along $\tilde{\vec{e}}$-direction on vortex-membrane with a plane Kelvin wave are given by $\vec{p}_{\text {Lamb }}=\mathbf{P}_{\text {Lamb }} \cdot \tilde{\vec{e}}= \pm \frac{1}{2} \rho \kappa V_{P} a^{2} \vec{k}$ and $\left|J_{\text {Lamb }}\right|=$ $\left|\mathbf{J}_{\text {Lamb }} \cdot \tilde{\vec{e}}\right|=\frac{1}{2} \rho \kappa V_{P} a^{2}$, respectively. $V_{p}$ is the total volume length of the vortexmembrane. Because the projected (Lamb impulse) angular momentum is a constant on the vortex-membrane, the effective Planck constant $\hbar_{\text {knot }}$ is derived as angular momentum in extra space that is proportional to the volume of the knot in $5 \mathrm{D}$ space, i.e., $\hbar_{\text {eff }}=J_{\text {Lamb }}=\frac{1}{2} \rho_{0} \kappa V_{P} r_{0}^{2}$, where $V_{p}$ is the total volume of the vortexmembrane and $\rho_{0}$ is the SF mass density.

For two entangled vortex-membranes, the nonlocal interaction leads to leapfrogging motion. ${ }^{10}$ For leapfrogging motion, the entangled vortex-membranes exchange energy in a periodic fashion. The winding radii of two vortex-membranes oscillate with a fixed leapfrogging angular frequency $\omega^{*}=(\alpha \kappa \ln \epsilon) \frac{2}{r_{0}^{2}}$, where $r_{0}$ is the distance between two vortex-membranes.

A knot is an elementary entanglement between two vortex-membranes with fixed volume. On the one hand, a knot is $\pi$-phase changing - a sharp, time-independent, topological phase-changing, on the other hand, a knot has a phase angle. Quantum mechanics describes the dynamics of smooth, slow, nontopological phase-changing of knots. From the point of view of information, the elementary volume-changing with a zero is a knot. Under fixed-volume condition, the knot becomes fragmentized and obeys quantum mechanics rather than pseudo-quantum mechanics. This is the fundamental principle of quantum mechanics. The effective Planck constant $\hbar_{\text {knot }}$ is derived as angular momentum in extra space (the volume of the knot in 5D space):

$$
\hbar_{\mathrm{knot}}=\frac{1}{2} \rho_{0} \kappa V_{P} r_{0}^{2}
$$

where $V_{p}$ is the total volume of the vortex-membrane and $\rho_{0}$ is the SF mass density.

We pointed out that the function of a Kelvin wave with an extra fragmentized knot describes the distribution of the knot-pieces and $\mathrm{z}(\vec{x}, t)$ plays the role of the wavefunction in quantum mechanics as

$$
\frac{1}{\sqrt{V_{P}}} \frac{\mathrm{z}(\vec{x}, t)}{r_{0}}=\sqrt{\rho_{\mathrm{knot}}(x, t)} e^{i \Delta \phi(\vec{x}, t)} \Leftrightarrow \psi(\vec{x}, t) .
$$

The angle $\Delta \phi(\vec{x}, t)$ becomes the quantum phase angle of wavefunction, the knot density $\rho_{\text {knot }}=\left\langle\frac{\Delta \hat{K}}{\Delta V_{P}}\right\rangle$ becomes the probability density of knot-pieces $n_{\text {knot }}(\vec{x})$. For 
a plane wave, $\psi(\vec{x}, t)=\frac{1}{\sqrt{V_{P}}} e^{-i \omega \cdot t+i \vec{k} \cdot \vec{x}}$, the projected (Lamb impulse) energy of a knot is

$$
E_{\mathrm{knot}}=\hbar_{\mathrm{knot}} \omega
$$

and the projected (Lamb impulse) momentum of a knot is

$$
\vec{p}_{\mathrm{knot}}=\hbar_{\mathrm{knot}} \vec{k}
$$

where the effective Planck constant $\hbar_{\mathrm{knot}}$ is obtained as projected (Lamb impulse) angular momentum of a knot (the elementary volume-changing of two entangled vortex-membranes)

$$
\hbar_{\mathrm{knot}}=J_{\mathrm{knot}}=\frac{1}{2} \rho_{0} \kappa V_{P} r_{0}^{2} .
$$

In general, the energy and momentum for a knot are described by operators:

$$
E_{\mathrm{knot}} \rightarrow \hat{E}_{\mathrm{knot}}=i \hbar_{\mathrm{eff}} \frac{d}{d t}, \quad \vec{p}_{\mathrm{knot}} \rightarrow \hat{p}_{\mathrm{knot}}=-i \hbar_{\mathrm{eff}} \frac{d}{d \vec{x}}
$$

The energy-momentum relationship $E=H(\vec{p})$ becomes the equation of motion for wavefunction,

$$
i \hbar_{\mathrm{eff}} \frac{d}{d t} \psi(\vec{x})=\hat{H}_{\mathrm{knot}}(\hat{p}) \psi(\vec{x}) .
$$

We also use path integral formulation to describe quantum processes in knot physics. For a multi-knot system, the probability amplitude is defined by

$$
\left\langle t_{f}, \vec{x}_{M}^{\prime}, \ldots, \vec{x}_{2}^{\prime}, \vec{x}_{1}^{\prime} \mid t_{i}, \vec{x}_{M}, \ldots, \vec{x}_{2}, \vec{x}_{1}\right\rangle=\int \mathcal{D} \psi^{\dagger}(\vec{x}, t) \mathcal{D} \psi(\vec{x}, t) e^{i \mathcal{S}_{\mathrm{knot}} / \hbar_{\mathrm{eff}}}
$$

where $\mathcal{S}_{\text {knot }}=\sum_{\omega, \vec{p}} S_{\omega, \vec{p}}=\int \mathcal{L}_{\text {knot }} d t d^{3} x$ with $S_{\omega, \vec{p}}=\psi_{\vec{p}}^{\dagger}\left(i \hbar_{\mathrm{eff}} \omega(\vec{p})-H_{\mathrm{knot}}(\vec{p})\right) \psi_{\vec{p}}$ and $\mathcal{L}_{\text {knot }}=i \psi^{\dagger} \partial_{t} \psi-\hat{\mathcal{H}}_{\text {knot }}$.

However, there is an unsolved problem in Ref. 13: because free Dirac model is noninteracting, we do not know how to do a physical dynamic projection. In this paper, we develop an effective quantum gauge field theory and solve the above problem.

\section{Composite Knot-Crystal: Definition, Classification and Generalized Translation Symmetry}

In solid state physics, a basic theory is about atom-crystal and its lattices. The atom-crystal has its inherent symmetry, by which we may classify different types of symmetries (translational symmetry, rotation symmetry, mirror symmetry). For example, there are 230 distinct space groups in 3D space. In addition to simple crystal with monatomic lattices, there exist composite crystals with polyatomic lattices that have more than one type of atoms in a unit cell.

In Ref. 13, a periodic entanglement pattern between two vortex-membranes is called knot-crystal. The definition of a "knot-crystal" is based on periodic structures of knots that is similar to atom-crystal where the atoms form a periodic 


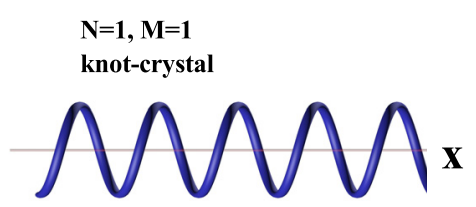

(a)
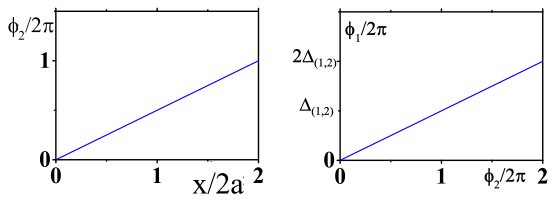

(b)

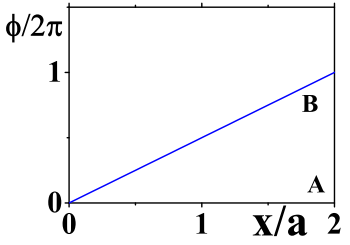

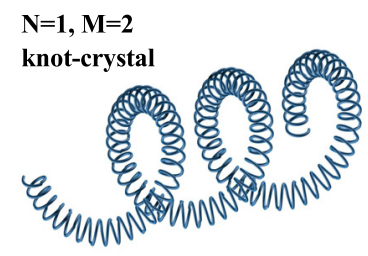

Fig. 2. (Color online) (a) An illustration of a knot-crystal with $(\mathcal{N}=1$ and $\mathcal{M}=1)$. The linear relationship between winding angle $\phi$ and $x$ indicates the generalized spatial translation symmetry; (b) an illustration of a composite knot-crystal with $(\mathcal{N}=1$ and $\mathcal{M}=2)$. There exists linear relationship between winding angle of level-2 Kelvin wave $\phi_{2}$ and $x$ and linear relationship between winding angle of level-1 Kelvin wave $\phi_{1}$ and winding angle of level-2 Kelvin wave $\phi_{2}$. In particular, level-2 vortex-membrane (that is a Kelvin wave) becomes center membrane of level-1 vortex-membrane.

arrangement. However, knot-crystal is different from traditional atom-crystal. Figure 2(a) shows a 1D knot-crystal in 3D space. Due to the existence of rotation symmetry and generalized translation symmetry, the properties of knot-crystals are much different from that of atom-crystals. In this paper, under LIA, we discuss the properties of composite knot-crystals, a periodic entanglement pattern between multi-vortex-membranes. The name of composite knot-crystal comes from the similarity to the polyatomic atom-crystal with a composite lattice.

\subsection{Definition}

Firstly, we consider an arbitrary $d$-dimensional composite knot-crystal that comes from a periodic entanglement pattern by entangled vortex-membranes in $d+2$ dimensional space $\left(x_{1}, x_{2}, \ldots, x_{d}, x_{d+1}, x_{d+2}\right)$. To characterize a composite knotcrystal, we define the function by an $\mathcal{N} \times \mathcal{M}$ matrix:

$$
\mathbf{Z}(\vec{x}, t)=\left(\begin{array}{c}
\mathbf{z}_{1}(\vec{x}, t) \\
\mathbf{z}_{2}(\vec{x}, t) \\
\ldots \\
\mathbf{z}_{\mathcal{N}}(\vec{x}, t)
\end{array}\right)=\left(\begin{array}{cccc}
z_{1,1}(\vec{x}, t) & z_{1,2}(\vec{x}, t) & \ldots & z_{1, \mathcal{M}}(\vec{x}, t) \\
z_{2,1}(\vec{x}, t) & z_{2,2}(\vec{x}, t) & \ldots & \ldots \\
\ldots & \ldots & \ldots & \ldots \\
z_{\mathcal{N}, 1}(\vec{x}, t) & z_{\mathcal{N}, 2}(\vec{x}, t) & \ldots & z_{\mathcal{N}, \mathcal{M}}(\vec{x}, t)
\end{array}\right),
$$

where $\vec{x}=\left(x_{1}, x_{2}, \ldots, x_{d}\right) . \mathcal{N}$ and $\mathcal{M}$ denote the vortex-index and the level-index, respectively. We point out that $\mathbf{Z}(\vec{x}, t)$ is just matrix representation to show the properties of knot-crystal and there exist $\mathcal{N} \times \mathcal{M}$ independent functions. $\mathbf{z}_{j}(\vec{x}, t)$ 
denotes the function of $j$ th vortex-membrane. A generalized definition of a knotcrystal is given by $\mathcal{N} \times \mathcal{M}$ independent functions of $j$ th level $i$ th vortex-membrane $z_{i, j}(\vec{x}, t)$ that is an element of the matrix as

$$
z_{i, j}(\vec{x}, t)=\prod_{I} r_{i, j}\left(\alpha_{i, j}^{I} e^{i \phi_{i, j}^{I}}+\beta_{i, j}^{I} e^{-i \phi_{i, j}^{I}}\right) e^{i \omega_{i, j} t+i\left(\phi_{i, j}^{I}\right)_{0}},
$$

where $\left|\alpha_{i, j}^{I}\right|^{2}+\left|\beta_{i, j}^{I}\right|^{2}=1$ and $I=x_{1}, x_{2}, \ldots, x_{d} \cdot \phi_{i, j}^{I}$ and $\left(\phi_{i, j}^{I}\right)_{0}$ denote the winding phase angle and the constant phase angle along the given $x^{I}$-direction $j$ th level $i$ th vortex-membrane, respectively. $\omega_{i, j}$ is rotating velocity and $r_{i, j}$ is the radius of $j$ th level $i$ th vortex-membrane, respectively. In general, to guarantee the stability of the composite knot-crystal, we consider LIA,

$$
r_{i, j} \gg r_{i, j-1} \text {. }
$$

In particular, $j$ th level $i$ th vortex-membrane (that is a Kelvin wave) becomes center membrane of $(j-1)$ th level $i$ th vortex-membrane.

To determine a composite knot-crystal, there is a hierarchy recurrence relationship between two nearest-neighbor levels along $x^{I}$-direction

$$
\begin{aligned}
\phi_{i, \mathcal{M}}^{I} & =k_{i, 0}^{I} \cdot x^{I}, \\
\phi_{i, \mathcal{M}-1}^{I} & =\Delta_{i,(\mathcal{M}-1, \mathcal{M})}^{I} \phi_{i, \mathcal{M}}^{I}, \\
& \cdots \\
\phi_{i, j-1}^{I} & =\Delta_{i,(j-1, j)}^{I} \phi_{i, j}^{I}, \\
& \cdots \\
\phi_{i, 1}^{I} & =\Delta_{i,(1,2)}^{I} \phi_{i, 2}^{I},
\end{aligned}
$$

where $k_{i, 0}^{I}=\frac{\pi}{a_{i, \mathcal{M}}^{I}}$ denotes winding vector along the winding direction, and $a_{i, \mathcal{M}}^{I}$ is the length that denotes the half pitch of largest windings of $i$ th vortex-membrane. Each number of the vector $\Delta_{i,(j-1, j)}^{I}$ is the positive winding number of $(j-1)$ th level $i$ th vortex-membrane on $j$ th level $i$ th vortex-membrane along $x^{I}$-direction. Thus, according to the hierarchy recurrence relationship, we have

$$
z_{i, j}(\vec{x}, t)=\prod_{I} r_{i, j}\left(\alpha_{i, j}^{I} e^{i \phi_{i, j}^{I}}+\beta_{i, j}^{I} e^{-i \phi_{i, j}^{I}}\right) e^{i \omega_{i, j} t+i\left(\phi_{i, j}^{I}\right)_{0}},
$$

where $\phi_{i, j}^{I}=\left[\prod_{j}^{\mathcal{M}} \Delta_{i,(j+1, j)}^{I}\right] \cdot \phi_{i, \mathcal{M}}^{I}$. As a result, the function for a knot-crystal with $\mathcal{M}$-level $\mathcal{N}$ vortex-membranes is described by the $\mathcal{N} \times \mathcal{M}$ winding angle $\phi_{i j}^{I}$,

$$
\mathbf{Z}\left(x^{I}, t\right)=\left(\begin{array}{cccc}
\prod_{I} z_{1,1}\left(\phi_{1,1}^{I}, t\right) & \prod_{I} z_{1,2}\left(\phi_{1,2}^{I}, t\right) & \ldots & \prod_{I} z_{1, \mathcal{M}}\left(\phi_{1, \mathcal{M}}^{I}, t\right) \\
\prod_{I} z_{2,1}\left(\phi_{2,1}^{I}, t\right) & \prod_{I} z_{2,2}\left(\phi_{2,2}^{I}, t\right) & \ldots & \ldots \\
\ldots & \ldots & \ldots & \ldots \\
\prod_{I} z_{\mathcal{N}, 1}\left(\phi_{\mathcal{N}, 1}^{I}, t\right) & \prod_{I} z_{\mathcal{N}, 2}\left(\phi_{\mathcal{N}, 2}^{I}, t\right) & \ldots & \prod_{I} z_{\mathcal{N}, \mathcal{M}}\left(\phi_{\mathcal{N}, \mathcal{M}}^{I}, t\right)
\end{array}\right)
$$


In this paper, we only consider the cases of

$$
\Delta_{i,(j-1, j)}^{I}=\Delta_{(j-1, j)}^{I} .
$$

As a result, the different vortex-membranes of the same level along different directions have the same winding length. The hierarchy series of the particular type of composite knot-crystal is given by

$$
\left\{\Delta_{(1,2)}, \Delta_{(2,3)}, \ldots, \Delta_{(\mathcal{M}-1, \mathcal{M})}\right\} .
$$

\subsection{Classification}

To classify a knot-crystal, we introduce three indices: dimension $d$, number of vortex-membranes $\mathcal{N}$, level $\mathcal{M}$. Each $d$-dimensional composite knot-crystal is denoted by $(\mathcal{N}, \mathcal{M})$.

The first index is dimension of knot-crystal, $d$. In this paper, we focus on 3D knot-crystal $(d=3)$ that is described by $\mathbf{Z}(\vec{x}, t)$, where $\vec{x}=(x, y, z)$. Figure 2 is an illustration of two types of $1 \mathrm{D}$ knot-crystals in $3 \mathrm{D}$ space.

The second index is the number of vortex-membranes, $\mathcal{N}$. For a $1 \mathrm{D}$ composite knot-crystal with $(\mathcal{N}=1, \mathcal{M})$, the function is

$$
\begin{aligned}
\mathbf{z}(x, t)= & \left(r_{1}\left(\alpha_{1} e^{i \phi_{1}}+\beta_{1} e^{-i \phi_{1}}\right) e^{i \omega_{1} t+i\left(\phi_{1}\right)_{0}}\right. \\
& \left.\cdots r_{\mathcal{M}}\left(\alpha_{\mathcal{M}} e^{i \phi_{\mathcal{M}}}+\beta_{\mathcal{M}} e^{-i \phi_{\mathcal{M}}}\right) e^{i \omega_{\mathcal{M}} t+i\left(\phi_{\mathcal{M}}\right)_{0}}\right)
\end{aligned}
$$

where $\left|\alpha_{i}\right|^{2}+\left|\beta_{i}\right|^{2}=1$ and $r_{i} \gg r_{i-1}$. Figure $2(\mathrm{~b})$ is an example of a $1 \mathrm{D}$ composite knot-crystal from one vortex-membrane of which the function is given by

$$
\mathbf{z}(x, t)=\left(r_{1} e^{i \phi_{1}(x)+i \omega_{1} t+i\left(\phi_{1}\right)_{0}} \quad r_{2} e^{i \phi_{2}(x)+i \omega_{2} t+i\left(\phi_{2}\right)_{0}}\right),
$$

where $r_{1} \ll r_{2}, \phi_{1}(x)=\Delta_{(1,2)} \cdot \phi_{2}(x)$ and $\Delta_{(1,2)}$ is a positive number.

The third index is level of knot-crystal, $\mathcal{M}$. To define the concept of level, we introduce composite knot-crystal that corresponds to polyatomic atom-crystal with a composite lattice. Figure 2(b) is an illustration of a 2-level knot-crystal with $(\mathcal{N}=1, \mathcal{M}=2)$. An important composite knot-crystal is standard knot-crystal with $(\mathcal{N}=4, \mathcal{M}=3)$ of which the hierarchy series along different directions is given by

$$
\left\{\Delta_{(1,2)}, \Delta_{(2,3)}\right\}
$$

where $\Delta_{(1,2)}=3$ and $\Delta_{(2,3)} \gg 1$.

In addition to the three indices, $d, \mathcal{N}, \mathcal{M}$, to characterize a composite knotcrystal, one need to define its tensor network state that denotes the entanglement pattern along different directions. In Ref. 13, we have studied a 3D simple knotcrystal with two vortex-membranes $(\mathcal{N}=2, \mathcal{M}=1)$ - spin-orbital coupling (SOC) knot-crystal that is characterized by the following tensor network state:

$$
\begin{aligned}
& \left\langle\sigma^{X} \otimes \overrightarrow{1}\right\rangle=\vec{n}_{\sigma}^{X}=(1,0,0), \\
& \left\langle\sigma^{Y} \otimes \overrightarrow{1}\right\rangle=\vec{n}_{\sigma}^{Y}=(0,1,0), \\
& \left\langle\sigma^{Z} \otimes \overrightarrow{1}\right\rangle=\vec{n}_{\sigma}^{Z}=(0,0,1) .
\end{aligned}
$$




\subsection{Generalized spatial translation symmetry}

One of the most important properties of a composite knot-crystal is the generalized spatial translation symmetry.

It is obvious that the knot-crystals break continuous translation symmetry, i.e.,

$$
\mathbf{Z}^{\prime}(\vec{x}, t) \neq \mathcal{T}\left(\delta x^{I} \rightarrow 0\right) \mathbf{Z}(\vec{x}, t),
$$

where $\mathbf{Z}(\vec{x}, t)$ is function of a knot-crystal and $\mathcal{T}\left(\delta x^{I}\right)$ is translation operator for knot-crystal. The knot-crystals have discrete translation symmetry as

$$
\mathbf{Z}^{\prime}(\vec{x}, t)=\mathcal{T}\left(\delta x^{I}=2 a^{I}\right) \mathbf{Z}(\vec{x}, t)=\mathbf{Z}(\vec{x}, t) .
$$

Here, $a^{I}$ is the length that denotes the half pitch of largest windings of vortexmembrane.

We point out that all knot-crystals have generalized spatial translation symmetry. To define the generalized translation symmetry for a knot-crystal, we do a translation operation, under which all vortex-membranes shift a distance $\left|\delta x^{I}\right|$ along the $x^{I}$-direction, i.e.,

$$
\begin{aligned}
\mathbf{Z}\left(x^{I}, t\right) & \rightarrow \mathbf{Z}^{\prime}\left(x^{I}, t\right) \\
& =\mathcal{T}\left(\delta x^{I}\right) \mathbf{Z}\left(x^{I}, t\right) \\
& =\left(\begin{array}{cccc}
z_{1,1}\left(\left(\phi_{1,1}^{I}+\delta \phi_{1,1}^{I}\right), t\right) & z_{1,2}\left(\left(\phi_{1,2}^{I}+\delta \phi_{1,2}^{I}\right), t\right) & \ldots & z_{1, \mathcal{M}}\left(\left(\phi_{1, \mathcal{M}}^{I}+\delta \phi_{1, \mathcal{M}}^{I}\right), t\right) \\
z_{2,1}\left(\left(\phi_{2,1}^{I}+\delta \phi_{2,1}^{I}\right), t\right) & z_{2,2}\left(\left(\phi_{2,2}^{I}+\delta \phi_{2,2}^{I}\right), t\right) & \ldots & \ldots \\
\ldots & \ldots & \ldots & \ldots \\
z_{\mathcal{N}, 1}\left(\left(\phi_{\mathcal{N}, 1}^{I}+\delta \phi_{\mathcal{N}, 1}^{I}\right), t\right) & z_{\mathcal{N}, 2}\left(\left(\phi_{\mathcal{N}, 2}^{I}+\delta \phi_{\mathcal{N}, 2}^{I}\right), t\right) & \ldots & z_{\mathcal{N}, \mathcal{M}}\left(\left(\phi_{\mathcal{N}, \mathcal{M}}^{I}+\delta \phi_{\mathcal{N}, \mathcal{M}}^{I}\right), t\right)
\end{array}\right) .
\end{aligned}
$$

Under the global generalized translation symmetry, we have

$$
\begin{aligned}
z_{i, j}\left(x^{I}, t\right) \rightarrow z_{i, j}^{\prime}\left(x^{I}, t\right) & =\mathcal{T}\left(\delta x^{I}\right) z_{i, j}\left(x^{I}, t\right)=\mathcal{T}\left(\delta x^{I}\right) z_{i, j}\left(\phi_{i, j}^{I}, t\right) \\
& =z_{i, j}\left(\left(\phi_{i, j}^{I}+\delta \phi_{i, j}^{I}\right), t\right), \quad j=1,2, \ldots \mathcal{M}
\end{aligned}
$$

where

$$
\delta \phi_{i, j-1}^{I}=\Delta_{i, j} \cdot \delta \phi_{i, j}^{I} .
$$

We can define generalized spatial translation symmetry for each level of composite knot-crystal, $\mathcal{T}_{l}\left(\delta x^{I}\right)$, i.e.,

$$
z_{i, j}(\vec{x}, t) \rightarrow z_{i, j}^{\prime}(\vec{x}, t)=\mathcal{T}_{l}\left(\delta x^{I}\right) z_{i, j}(\vec{x}, t)=z_{i, j}\left(\left(\phi_{i, j}^{I}+\delta_{l j} \delta \phi_{i, j}^{I}\right), t\right) .
$$

For the case of $l=j$, we have $\delta_{l j}=1$; for the case of $l \neq j$, we have $\delta_{l j}=0$.

\subsection{Examples of composite knot-crystals}

3.4.1. 1-level winding knot-crystal with $(\mathcal{N}=1, \mathcal{M}=1)$

Firstly, we consider the simplest knot-crystal - 1-level winding knot-crystal with $(\mathcal{N}=1, \mathcal{M}=1)$. 
A $3 \mathrm{D}$ winding knot-crystal with $(\mathcal{N}=1, \mathcal{M}=1)$ is just a pure state of Kelvin wave from a vortex-membrane in $5 \mathrm{D}$ space $(x, y, z, \xi, \eta)$ that is described by

$$
\mathrm{z}\left(x^{I}, t\right)=r_{0}\left(\alpha e^{i \phi\left(x^{I}\right)}+\beta e^{-i \phi\left(x^{I}\right)}\right) e^{i \omega t+i \phi_{0}},
$$

where $x^{I}$ is the winding coordinate along $\vec{e}^{I}$-direction.

To distinguish the traveling Kelvin wave and standing Kelvin wave, we have introduced the spin network representation (a reduction representation of tensor network state) of Kelvin waves. ${ }^{13}$ Different spin network states of Kelvin waves have different spin directions:

$$
\left\langle\sigma^{I}\right\rangle=\langle\mathrm{z}]\left(\sigma^{I}\right)[\mathrm{z}\rangle=\vec{n}_{\sigma}^{I}, \quad I=x, y, z,
$$

where $\sigma^{I}$ is $2 \times 2$ Pauli matrix for helical degree of freedom. Different knot-crystals with $(\mathcal{N}=1, \mathcal{M}=1)$ are characterized by different spin network states $\vec{n}_{\sigma}$.

\subsubsection{1-level knot-crystal with $(\mathcal{N}=2, \mathcal{M}=1)$}

We then review the properties of another simple knot-crystal - 3D 1-level knotcrystal with $(\mathcal{N}=2, \mathcal{M}=1)$ (two entangled vortex-membranes) in $5 \mathrm{D}$ space $(x, y, z, \xi, \eta)$ that is described by $\mathbf{Z}(\vec{x}, t)=\left(\begin{array}{l}\mathrm{z}_{\mathrm{A}}(\vec{x}, t) \\ \mathrm{z}_{\mathrm{B}}(\vec{x}, t)\end{array}\right)$.

To distinguish the traveling Kelvin wave and standing Kelvin wave, we have introduced the tensor network representation of Kelvin waves. Different tensor states of Kelvin waves have different tensor network:

$$
\left\langle\sigma^{I} \otimes \tau^{I}\right\rangle=\langle\mathbf{Z}]\left(\sigma^{I} \otimes \tau^{I}\right)[\mathbf{Z}\rangle=\vec{n}_{\sigma}^{I} \otimes \overrightarrow{1}, \quad I=x, y, z,
$$

where $\sigma^{I}, \tau^{I}$ are $2 \times 2$ Pauli matrices for helical and vortex degrees of freedom, respectively. An interesting type of knot-crystal is SOC knot-crystal that is characterized by the tensor network states

$$
\begin{aligned}
& \left\langle\sigma^{X} \otimes \overrightarrow{1}\right\rangle=\vec{n}_{\sigma}^{X}=(1,0,0), \\
& \left\langle\sigma^{Y} \otimes \overrightarrow{1}\right\rangle=\vec{n}_{\sigma}^{Y}=(0,1,0), \\
& \left\langle\sigma^{Z} \otimes \overrightarrow{1}\right\rangle=\vec{n}_{\sigma}^{Z}=(0,0,1) .
\end{aligned}
$$

There always exists leapfrogging motion for the two entangled vortexmembranes with a fixed leapfrogging angular frequency $\omega^{*}$ and fixed distance $r_{0}$. As a result, along $x$-direction, the function of the Kelvin waves becomes

$$
\frac{r_{0}}{\sqrt{2}}\left(\begin{array}{c}
1+e^{i \omega^{*} t} \\
1-e^{i \omega^{*} t}
\end{array}\right) \cos (k \cdot x) e^{-i \omega_{0} t} ;
$$

along $y$-direction, the function becomes

$$
\frac{r_{0}}{\sqrt{2}}\left(\begin{array}{c}
1+e^{i \omega^{*} t} \\
1-e^{i \omega^{*} t}
\end{array}\right) e^{-i \omega_{0} t} \cdot\left(e^{i k \cdot y}+i e^{-i k \cdot y}\right) ;
$$




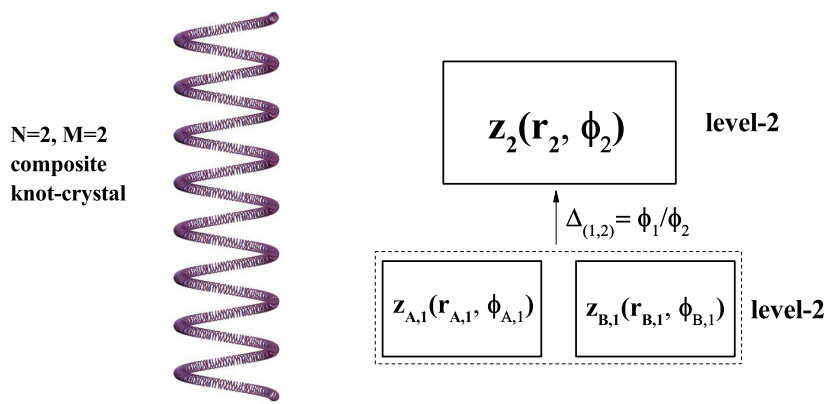

(a)

(b)

Fig. 3. (Color online) (a) An illustration of a 1D composite knot-crystal with $(\mathcal{N}=2, \mathcal{M}=2)$ (a system of two entangled vortex-lines); (b) the hierarchy structure of a 1D composite knot-crystal with $(\mathcal{N}=2, \mathcal{M}=2)$.

along $z$-direction, the function becomes

$$
r_{0}\left(\begin{array}{c}
1+e^{i \omega^{*} t} \\
1-e^{i \omega^{*} t}
\end{array}\right) e^{i k \cdot z} e^{-i \omega_{0} t} .
$$

\subsubsection{2-level composite winding knot-crystal with $(\mathcal{N}=2, \mathcal{M}=2)$}

A 2-level composite winding knot-crystal is an object of two entangled vortexmembranes A and B. To generate a 2-level winding knot-crystal, we firstly tangle two symmetric vortex-membranes $\mathrm{A}$ and $\mathrm{B}$ and get a knot-crystal. Next, we wind the knot-crystal and get a 2-level composite knot-crystal with $(\mathcal{N}=2, \mathcal{M}=2)$. See the illustration in Fig. 3(a). In Fig. 3(b), we show the hierarchy structure of a composite knot-crystal with $(\mathcal{N}=2, \mathcal{M}=2)$.

(a) The definition: In general, the function of 2-level composite knot-crystal with $(\mathcal{N}=2, \mathcal{M}=2)$ is denoted by

$$
\mathbf{Z}\left(x^{I}, t\right)=\left(\begin{array}{ll}
\prod_{I} z_{\mathrm{A}, 1}\left(\phi_{\mathrm{A}, 1}^{I}, t\right) & \prod_{I} z_{\mathrm{A}, 2}\left(\phi_{\mathrm{A}, 2}^{I}, t\right) \\
\prod_{I} z_{\mathrm{B}, 1}\left(\phi_{\mathrm{B}, 1}^{I}, t\right) & \prod_{I} z_{\mathrm{B}, 2}\left(\phi_{\mathrm{B}, 2}^{I}, t\right)
\end{array}\right) .
$$

According to $\phi_{\mathrm{A}, 2}^{I}\left(x^{I}\right)=\phi_{\mathrm{B}, 2}^{I}\left(x^{I}\right)=\phi_{2}^{I}\left(x^{I}, t\right)$ or

$$
\prod_{I} z_{\mathrm{A}, 2}\left(\phi_{\mathrm{A}_{1}, 2}^{I}\left(x^{I}\right), t\right)=\prod_{I} z_{\mathrm{B}, 2}\left(\phi_{\mathrm{B}_{1}, 2}^{I}\left(x^{I}\right), t\right)=\prod_{I} \mathrm{z}_{2}\left(\phi_{2}^{I}\left(x^{I}, t\right), t\right)=\mathrm{z}_{2}(\vec{x}, t),
$$

$\mathrm{z}_{2}(\vec{x}, t)$ denotes the center membrane of vortex-membrane-A and vortex-membraneB. $\mathrm{z}_{\mathrm{A}, 1}(\vec{x}, t)=\prod_{I} z_{\mathrm{A}, 1}\left(\phi_{\mathrm{A}, 1}^{I}, t\right)$ denotes the vortex-membrane-A and $\mathrm{z}_{\mathrm{B}, 1}(\vec{x}, t)=$ $\prod_{I} z_{\mathrm{B}, 1}\left(\phi_{\mathrm{B}, 1}^{I}, t\right)$ denotes the vortex-membrane-B. The phase angles of $\mathrm{z}_{2}\left(x^{I}, t\right)$ are 
$\phi_{2}^{I}\left(x^{I}, t\right)$ and the winding radius of $\mathrm{z}_{2}(\vec{x}, t)$ is $r_{2}(\vec{x}, t)$. The winding radii $r_{\mathrm{A} / \mathrm{B}, 1}$ are the winding radii of vortex-membranes $\mathbf{z}_{\mathrm{A} / \mathrm{B}, 1}(\vec{x}, t)$ around its center membrane; $\phi_{\mathrm{A} / \mathrm{B}, 1}^{I}\left(x^{I}, t\right)$ are the phase angles of vortex-membranes-A/B. In particular, we consider a perturbative condition,

$$
r_{2} \gg r_{\mathrm{A} / \mathrm{B}, 1} \text {. }
$$

The hierarchy series is just a number

$$
\Delta_{(1,2)}=\frac{\phi_{\mathrm{A}, 1}^{I}\left(x^{I}, t\right)}{\phi_{2}^{I}\left(x^{I}, t\right)}=\frac{\phi_{\mathrm{B}, 1}^{I}\left(x^{I}, t\right)}{\phi_{2}^{I}\left(x^{I}, t\right)} .
$$

(b) Example: In this paper, we focus on a particular type of 2-level composite knot-crystal with $(\mathcal{N}=2, \mathcal{M}=2) \cdot\left(\begin{array}{l}\mathrm{z}_{\mathrm{A}, 1}(\vec{x}, t) \\ \mathrm{z}_{\mathrm{B}, 1}(\vec{x}, t)\end{array}\right)$ becomes the function of an SOC knot-crystal with leapfrogging motion. The tensor network state of $\left(\begin{array}{l}\mathrm{z}_{\mathrm{A}, 1}(\vec{x}, t) \\ \mathrm{z}_{\mathrm{B}, 1}(\vec{x}, t)\end{array}\right)$ is

$$
\begin{aligned}
& \left\langle\sigma^{X} \otimes \overrightarrow{1}\right\rangle=\vec{n}_{\sigma}^{X}=(1,0,0), \\
& \left\langle\sigma^{Y} \otimes \overrightarrow{1}\right\rangle=\vec{n}_{\sigma}^{Y}=(0,1,0), \\
& \left\langle\sigma^{Z} \otimes \overrightarrow{1}\right\rangle=\vec{n}_{\sigma}^{Z}=(0,0,1) .
\end{aligned}
$$

$\mathrm{z}_{2}(\vec{x}, t)$ is effective $\sigma_{z}$-type of knot-crystal. The tensor network state is

$$
\begin{aligned}
& \left\langle\sigma^{X} \otimes \overrightarrow{1}\right\rangle=\vec{n}_{\sigma}^{X}=(1,0,0), \\
& \left\langle\sigma^{Y} \otimes \overrightarrow{1}\right\rangle=\vec{n}_{\sigma}^{Y}=(1,0,0), \\
& \left\langle\sigma^{Z} \otimes \overrightarrow{1}\right\rangle=\vec{n}_{\sigma}^{Z}=(1,0,0) .
\end{aligned}
$$

In this paper, the hierarchy series $\Delta_{(1,2)}$ is considered to be a large positive integer number.

(c) Twist-writhe locking condition: We then introduce a topological constraint condition for a 2-level composite knot-crystal - twist-writhe locking condition.

To characterize a 2-level composite knot-crystal with $(\mathcal{N}=2, \mathcal{M}=2)$, we introduce three types of $1 \mathrm{D}$ translation symmetry protected topological invariable: linking number, writhe number, and twist number. So, there are three types of vectors for topological numbers to describe entanglement between two vortex-membranes: a $1 \mathrm{D}$ linking number density vector, a writhe number density vector, a twist number density vector. We point out that there exists an important topological relationship between these topological numbers - the twist-writhe locking condition.

Firstly, we discuss the entanglement between two entangled vortex-membranes for 2-level composite knot-crystal with $(\mathcal{N}=2, \mathcal{M}=2)\left(\mathrm{z}_{\mathrm{A}, 1}(\vec{x}, t)\right.$ and $\left.\mathrm{z}_{\mathrm{B}, 1}(\vec{x}, t)\right)$ of which the vector of (translation symmetry protected) $1 \mathrm{D}$ linking numbers $\boldsymbol{\zeta}_{(\mathrm{AB}), 1 \mathrm{D}}=\left(\zeta_{(\mathrm{AB}), 1 \mathrm{D}}^{x}, \zeta_{(\mathrm{AB}), 1 \mathrm{D}}^{y}, \zeta_{(\mathrm{AB}), 1 \mathrm{D}}^{z}\right), \frac{13}{1}$ where

$$
\zeta_{(\mathrm{AB}), 1 \mathrm{D}}^{I}=\frac{1}{4 \pi} \oint_{C_{x^{I}, \mathrm{~A}}} \oint_{C_{x^{I}, \mathrm{~B}}} \frac{\left(\mathbf{s}_{\mathrm{A}}^{I}-\mathbf{s}_{\mathrm{B}}^{I}\right) \cdot d \mathbf{s}_{\mathrm{A}}^{I} \times d \mathbf{s}_{\mathrm{B}}^{I}}{\left|\mathbf{s}_{\mathrm{A}}^{I}-\mathbf{s}_{\mathrm{B}}^{I}\right|^{3}} .
$$


Here, $\mathbf{s}_{\mathrm{A} / \mathrm{B}}^{I}=\mathbf{r} \cdot \vec{e}^{I}$ is the spatial vector of vortex-membranes along a given direction $\vec{e}^{I}(I=x, y, z)$. We decompose the linking number $\zeta_{(\mathrm{AB}), 1 \mathrm{D}}^{I}$ into the vector of writhe number $W_{(\mathrm{AB}), 1 \mathrm{D}}^{I}=W_{1 \mathrm{D}, \mathrm{A}}^{I}+W_{1 \mathrm{D}, \mathrm{B}}^{I}$, and the vector of the twist number $T_{(\mathrm{AB}), 1 \mathrm{D}}^{I}=T_{1 \mathrm{D}, \mathrm{A}}^{I}+T_{1 \mathrm{D}, \mathrm{B}}^{I}$, i.e., $\zeta_{(\mathrm{AB}), 1 \mathrm{D}}^{I}=W_{(\mathrm{AB}), 1 \mathrm{D}}^{I}+T_{(\mathrm{AB}), 1 \mathrm{D}}^{I}$, where

$$
\begin{aligned}
W_{(\mathrm{AB}), 1 \mathrm{D}}^{I}= & \frac{1}{4 \pi} \oint_{C_{x^{I}, \mathrm{~A}}} \oint_{C_{x^{I}, \mathrm{~A}}} \frac{\left(\mathbf{s}_{1}^{I}-\mathbf{s}_{2}\right) \cdot d \mathbf{s}_{1}^{I} \times d \mathbf{s}_{2}}{\left|\mathbf{s}_{1}^{I}-\mathbf{s}_{2}\right|^{3}} \\
& +\frac{1}{4 \pi} \oint_{C_{x^{I}, \mathrm{~B}}} \oint_{C_{x^{I}, \mathrm{~B}}} \frac{\left(\mathbf{s}_{1}^{I}-\mathbf{s}_{2}\right) \cdot d \mathbf{s}_{1}^{I} \times d \mathbf{s}_{2}}{\left|\mathbf{s}_{1}^{I}-\mathbf{s}_{2}\right|^{3}}, \\
T_{(\mathrm{AB}), 1 \mathrm{D}}^{I}= & \frac{1}{2 \pi} \oint_{C_{x^{I}, \mathrm{~A}}}\left(\mathbf{N}^{I} \times\left(\mathbf{N}^{I}\right)^{\prime}\right) \cdot d \mathbf{s}^{I} \\
& +\frac{1}{2 \pi} \oint_{C_{x^{I}, \mathrm{~B}}}\left(\mathbf{N}^{I} \times\left(\mathbf{N}^{I}\right)^{\prime}\right) \cdot d \mathbf{s}^{I},
\end{aligned}
$$

where a unit spanwise vector $\mathbf{N}^{I}=\hat{\mathbf{n}}^{I} \cos \theta^{I}+\hat{\mathbf{b}}^{I} \sin \theta^{I}$ determines the twisting of the vortex-membranes along $x^{I}$-direction. $\hat{\mathbf{n}}^{I}$ and $\hat{\mathbf{b}}^{I}$ are local normal and bi-normal (unit vectors) of the vortex-membrane in 5D fluid along $x^{I}$-direction, respectively ( $\theta^{I}$ is the corresponding mixing angle).

Because the linking number is a topological-invariant for two entangled vortexmembranes, we have a topological constraint condition - twist-writhe locking condition, ${ }^{15,16}$

$$
\zeta_{(\mathrm{AB}), 1 \mathrm{D}}^{I}=W_{(\mathrm{AB}), 1 \mathrm{D}}^{I}+T_{(\mathrm{AB}), 1 \mathrm{D}}^{I} \equiv \text { const. }, \quad(I=x, y, z) .
$$

Theoretically under continuous deformation of the vortex-membranes, the writhe number $W_{(\mathrm{AB}), 1 \mathrm{D}}^{I}$ and the twist number $T_{(\mathrm{AB}), 1 \mathrm{D}}^{I}$ vary together, i.e.,

$$
\delta W_{(\mathrm{AB}), 1 \mathrm{D}}^{I} \equiv-\delta T_{(\mathrm{AB}), 1 \mathrm{D}}^{I}, \quad(I=x, y, z) .
$$

From the above discussion, we point out that for two entangled vortexmembranes (2-level composite knot-crystal with $(\mathcal{N}=2, \mathcal{M}=2)$ ) when the two vortex-membranes have additional global winding, finite $\delta W_{(\mathrm{AB}), 1 \mathrm{D}}^{I}$ leads to finite $-\delta T_{(\mathrm{AB}), 1 \mathrm{D}}^{I}$ that is really an additional entanglement between two vortexmembranes and vice versa.

It was known that the vector of linking number density operators for two entangled vortex-membranes (2-level composite knot-crystal with $(\mathcal{N}=2, \mathcal{M}=2)$ ) are defined by

$$
\hat{\rho}_{\text {linking }}=\left(-\frac{i}{2 \pi r_{0}^{2}} \frac{d}{d x^{x}},-\frac{i}{2 \pi r_{0}^{2}} \frac{d}{d x^{y}},-\frac{i}{2 \pi r_{0}^{2}} \frac{d}{d x^{z}}\right),
$$

respectively. For a 2-level composite knot-crystal with $(\mathcal{N}=2, \mathcal{M}=2)$, the three $1 \mathrm{D}$ (spatial translation symmetry protected) linking numbers $\zeta_{1 \mathrm{D}}^{I}(I=x, y, z)$ are conserved. 


\section{S.-P. Kou}

For 3D 2-level composite knot-crystal with $(\mathcal{N}=2, \mathcal{M}=2)$ in $5 \mathrm{D}$ space, we define the (spatial translation symmetry protected) 1D writhe density vector

$$
\rho_{\mathrm{writhe}}^{I}\left(x^{I}, t\right)=\frac{d W_{1 \mathrm{D}}^{I}}{d x^{I}}
$$

and the (spatial translation symmetry protected) 1D twist density vector

$$
\rho_{\text {twist }}^{I}\left(x^{I}, t\right)=\frac{d T_{1 \mathrm{D}}^{I}}{d x^{I}} .
$$

Due to the twist-writhe locking condition and spatial translation symmetry, for 2level composite knot-crystal with $(\mathcal{N}=2, \mathcal{M}=2)$, we have the following equation:

$$
\rho_{\text {linking }}^{I}\left(x^{I}, t\right)=\rho_{\text {writhe }}^{I}\left(x^{I}, t\right)+\rho_{\text {twist }}^{I}\left(x^{I}, t\right), \quad(I=x, y, z) .
$$

\subsubsection{2-level composite double-helix knot-crystal with $(\mathcal{N}=4, \mathcal{M}=2)$}

A 2-level composite knot-crystal with $(\mathcal{N}=4, \mathcal{M}=2)$ is an object of four entangled vortex-membranes $\mathrm{A}_{1}, \mathrm{~A}_{2}$ and $\mathrm{B}_{1}, \mathrm{~B}_{2}$. To generate a 2-level composite knotcrystal with $(\mathcal{N}=4, \mathcal{M}=2)$, we firstly tangle two symmetric vortex-membranes $A_{1}$ and $A_{2}$ and get A-knot-crystal. Next, we tangle two symmetric vortexmembranes $\mathrm{B}_{1}$ and $\mathrm{B}_{2}$ and get B-knot-crystal. Then, we tangle A-knot-crystal and B-knot-crystal into a composite knot-crystal. See the illustration in Fig. 4(a). In Fig. 4(b), we show the hierarchy structure of a composite knot-crystal with $(\mathcal{N}=4, \overline{\mathcal{M}}=2)$.

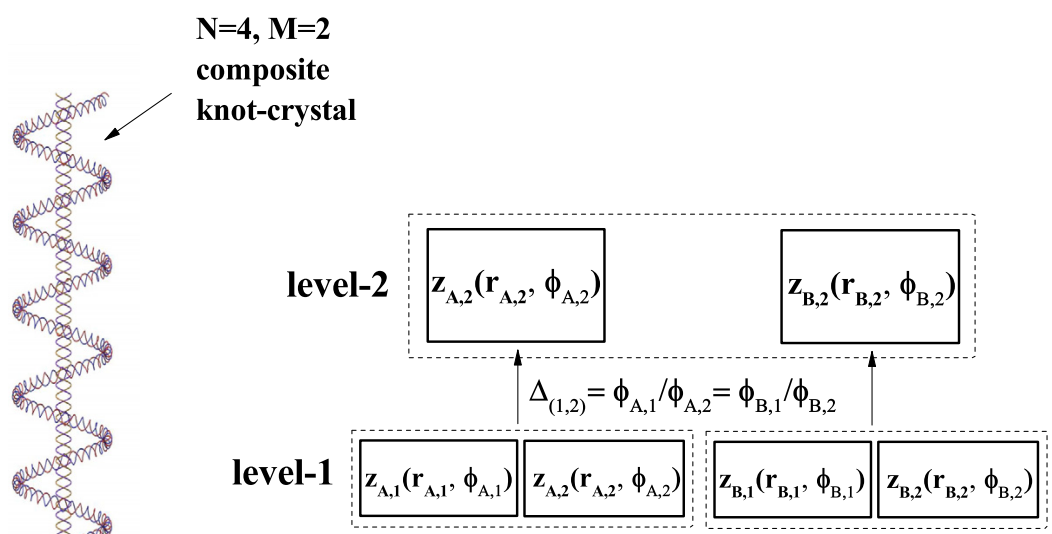

(b)

Fig. 4. (Color online) (a) An illustration of a $1 \mathrm{D}$ composite knot-crystal with $(\mathcal{N}=4, \mathcal{M}=2)$ (a system of four entangled vortex-lines); (b) the hierarchy structure of a 1D composite knotcrystal with $(\mathcal{N}=4, \mathcal{M}=2)$. 
(a) The definition: In general, the function of 2-level composite knot-crystal with $(\mathcal{N}=4, \mathcal{M}=2)$ is denoted by

$$
\mathbf{Z}(\vec{x}, t)=\left(\begin{array}{ll}
\prod_{I} z_{\mathrm{A}_{1}, 1}\left(\phi_{\mathrm{A}_{1}, 1}^{I}, t\right) & \prod_{I} z_{\mathrm{A}_{1}, 2}\left(\phi_{\mathrm{A}_{1}, 2}^{I}, t\right) \\
\prod_{I} z_{\mathrm{A}_{2}, 1}\left(\phi_{\mathrm{A}_{2}, 1}^{I}, t\right) & \prod_{I} z_{\mathrm{A}_{2}, 2}\left(\phi_{\mathrm{A}_{2}, 2}^{I}, t\right) \\
\prod_{I} z_{\mathrm{B}_{1}, 1}\left(\phi_{\mathrm{B}_{1}, 1}^{I}, t\right) & \prod_{I} z_{\mathrm{B}_{1}, 2}\left(\phi_{\mathrm{B}_{1}, 2}^{I}, t\right) \\
\prod_{I} z_{\mathrm{B}_{2}, 1}\left(\phi_{\mathrm{B}_{2}, 1}^{I}, t\right) & \prod_{I} z_{\mathrm{B}_{2}, 2}\left(\phi_{\mathrm{B}_{2}, 2}^{I}, t\right)
\end{array}\right) .
$$

According to $\phi_{\mathrm{A}_{1}, 2}^{I}\left(x^{I}\right)=\phi_{\mathrm{A}_{2}, 2}^{I}\left(x^{I}\right)=\phi_{\mathrm{A}, 2}^{I}\left(x^{I}, t\right)$ and $\phi_{\mathrm{B}_{1}, 2}^{I}\left(x^{I}\right)=\phi_{\mathrm{B}_{2}, 2}^{I}\left(x^{I}\right)=$ $\phi_{\mathrm{B}, 2}^{I}\left(x^{I}, t\right)$ or

$$
\prod_{I} z_{\mathrm{A}_{1}, 2}\left(\phi_{\mathrm{A}_{1}, 2}^{I}\left(x^{I}\right), t\right)=\prod_{I} z_{\mathrm{A}_{2}, 2}\left(\phi_{\mathrm{A}_{2}, 2}^{I}\left(x^{I}\right), t\right)=\mathrm{z}_{\mathrm{A}, 2}(\vec{x}, t)
$$

and

$$
\prod_{I} z_{\mathrm{B}_{1}, 2}\left(\phi_{\mathrm{B}_{1}, 2}^{I}\left(x^{I}\right), t\right)=\prod_{I} z_{\mathrm{B}_{2}, 2}\left(\phi_{\mathrm{B}_{2}, 2}^{I}\left(x^{I}\right), t\right)=\mathrm{z}_{\mathrm{B}, 2}(\vec{x}, t),
$$

$\mathbf{Z}_{2}(\vec{x}, t)=\left(\begin{array}{l}\mathrm{z}_{\mathrm{A}, 2}(\vec{x}, t) \\ \mathrm{z}_{\mathrm{B}, 2}(\vec{x}, t)\end{array}\right)$ denote the center membranes of A-knot-crystal and B-knotcrystal, respectively, or, $\mathrm{z}_{\mathrm{A}, 2}(\vec{x}, t)$ denotes the global position of the entangled vortex-membranes $\mathrm{A}_{1}, \mathrm{~A}_{2}$ and $\mathrm{z}_{\mathrm{B}, 2}(\vec{x}, t)$ denotes the global position of the entangled vortex-membranes $\mathrm{B}_{1}, \mathrm{~B}_{2}$.

$$
\begin{aligned}
& \mathbf{Z}_{\mathrm{A}, 1}^{\prime}(\vec{x}, t)=\left(\begin{array}{c}
z_{\mathrm{A}_{1}, 1}(\vec{x}, t) \\
z_{\mathrm{A}_{2}, 1}(\vec{x}, t)
\end{array}\right)=\left(\begin{array}{l}
\prod_{I} z_{\mathrm{A}_{1}, 1}\left(\phi_{\mathrm{A}_{1}, 1}^{I}\left(\phi_{\mathrm{A}_{1}, 2}^{I}\left(x^{I}\right)\right), t\right) \\
\prod_{I} z_{\mathrm{A}_{2}, 1}\left(\phi_{\mathrm{A}_{2}, 1}^{I}\left(\phi_{\mathrm{A}_{1}, 2}^{I}\left(x^{I}\right)\right), t\right)
\end{array}\right) \\
& \mathbf{Z}_{\mathrm{B}, 1}^{\prime}(\vec{x}, t)=\left(\begin{array}{c}
z_{\mathrm{B}_{1}, 1}(\vec{x}, t) \\
z_{\mathrm{B}_{2}, 1}(\vec{x}, t)
\end{array}\right)=\left(\begin{array}{l}
\prod_{I} z_{\mathrm{B}_{1}, 1}\left(\phi_{\mathrm{B}_{1}, 1}^{I}\left(\phi_{\mathrm{B}_{1}, 2}^{I}\left(x^{I}\right)\right), t\right) \\
\prod_{I} z_{\mathrm{B}_{2}, 1}\left(\phi_{\mathrm{B}_{2}, 1}^{I}\left(\phi_{\mathrm{B}_{1}, 2}^{I}\left(x^{I}\right)\right), t\right)
\end{array}\right)
\end{aligned}
$$

denote local entanglement between two vortex-membranes $\mathrm{A}_{1}, \mathrm{~A}_{2}$ and $\mathrm{B}_{1}, \mathrm{~B}_{2}$, respectively. The phase angles of $\mathrm{z}_{\mathrm{A} / \mathrm{B}, 2}\left(x^{I}, t\right)$ are $\phi_{\mathrm{A} / \mathrm{B}, 2}^{I}\left(x^{I}, t\right)$ and the winding radii of $\mathrm{z}_{\mathrm{A} / \mathrm{B}, 2}(\vec{x}, t)$ are $r_{\mathrm{A} / \mathrm{B}, 2}(\vec{x}, t)$. The winding radii $r_{\mathrm{A}_{i} / \mathrm{B}_{i}, 1}$ are the local winding radii of vortex-membranes $\mathbf{Z}_{\mathrm{A}_{i} / \mathrm{B}_{i}, 1}^{\prime}(\vec{x}, t)$ around its center; $\phi_{\mathrm{A}_{i} / \mathrm{B}_{i}, 1}^{I}\left(x^{I}, t\right)$ are the angles of vortex-membranes $\mathbf{Z}_{\mathrm{A}_{i} / \mathrm{B}_{i}, 1}^{\prime}(\vec{x}, t)$ around its center-membrane $\mathrm{A} / \mathrm{B}$. Then, to characterize a knot on winding entangled vortex-membranes, we need two types of phase angles - one is $\phi_{\mathrm{A} / \mathrm{B}, 2}^{I}(\vec{x}, t)$ that describe the winding position of the knot on center membrane $\mathrm{z}_{\mathrm{A} / \mathrm{B}, 2}(\vec{x}, t)$, the other is $\phi_{\mathrm{A}_{i} / \mathrm{B}_{i}, 1}^{I}\left(x^{I}, t\right)$ that are the phase angles of internal windings. 
In particular, we consider a perturbative condition,

$$
r_{2}=\sqrt{r_{\mathrm{A}, 2}^{2}(\vec{x}, t)+r_{\mathrm{B}, 2}^{2}(\vec{x}, t)} \gg\left|r_{\mathrm{A}_{i} / \mathrm{B}_{i}, 1}(\vec{x}, t)\right| .
$$

The hierarchy series is also a number

$$
\Delta_{(1,2)}=\frac{\phi_{\mathrm{A}_{1}, 1}^{I}\left(x^{I}, t\right)}{\phi_{\mathrm{A}, 2}^{I}\left(x^{I}, t\right)}=\frac{\phi_{\mathrm{B}_{1}, 1}^{I}\left(x^{I}, t\right)}{\phi_{\mathrm{B}, 2}^{I}\left(x^{I}, t\right)}=\frac{\phi_{\mathrm{A}_{2}, 1}^{I}\left(x^{I}, t\right)}{\phi_{\mathrm{A}, 2}^{I}\left(x^{I}, t\right)}=\frac{\phi_{\mathrm{B}_{2}, 1}^{I}\left(x^{I}, t\right)}{\phi_{\mathrm{B}, 2}^{I}\left(x^{I}, t\right)} .
$$

(b) Example: In this paper, we focus on a particular type of 2-level composite knot-crystal with $(\mathcal{N}=4, \mathcal{M}=2)$. $\mathbf{Z}_{2}(\vec{x}, t)$ becomes an effective SOC knot-crystal, i.e.,

$$
\mathbf{Z}_{2}(\vec{x}, t)=\left(\begin{array}{l}
\mathrm{z}_{\mathrm{A}, 2}(\vec{x}, t) \\
\mathrm{z}_{\mathrm{B}, 2}(\vec{x}, t)
\end{array}\right) .
$$

The tensor network state of $\mathbf{Z}_{2}(\vec{x}, t)$ is also

$$
\begin{aligned}
& \left\langle\sigma^{X} \otimes \overrightarrow{1}\right\rangle=\vec{n}_{\sigma}^{X}=(1,0,0), \\
& \left\langle\sigma^{Y} \otimes \overrightarrow{1}\right\rangle=\vec{n}_{\sigma}^{Y}=(0,1,0), \\
& \left\langle\sigma^{Z} \otimes \overrightarrow{1}\right\rangle=\vec{n}_{\sigma}^{Z}=(0,0,1) .
\end{aligned}
$$

$\mathbf{Z}_{\mathrm{A}, 1}^{\prime}(\vec{x}, t)$ and $\mathbf{Z}_{\mathrm{B}, 1}^{\prime}(\vec{x}, t)$ are effective SOC knot-crystal. The tensor network state is

$$
\begin{aligned}
& \left\langle\sigma^{X} \otimes \overrightarrow{1}\right\rangle=\vec{n}_{\sigma}^{X}=(1,0,0), \\
& \left\langle\sigma^{Y} \otimes \overrightarrow{1}\right\rangle=\vec{n}_{\sigma}^{Y}=(1,0,0), \\
& \left\langle\sigma^{Z} \otimes \overrightarrow{1}\right\rangle=\vec{n}_{\sigma}^{Z}=(1,0,0) .
\end{aligned}
$$

The hierarchy series $\Delta_{(1,2)}$ is considered to be a positive integer number $n$ (for example, $n=3)$.

(c) Twist-writhe locking condition: We have discussed the twist-writhe locking relation for a composite knot-crystal (vortex-membrane-A and vortex-membrane-B) and get

$$
\zeta_{(\mathrm{AB}), 1 \mathrm{D}}^{I}=W_{(\mathrm{AB}), 1 \mathrm{D}}^{I}+T_{(\mathrm{AB}), 1 \mathrm{D}}^{I} \equiv \text { const. }, \quad(I=x, y, z),
$$

where $\zeta_{(\mathrm{AB}), 1 \mathrm{D}}^{I}, W_{(\mathrm{AB}), 1 \mathrm{D}}^{I}$ and $T_{(\mathrm{AB}), 1 \mathrm{D}}^{I}$ are linking number writhe number and twist number, respectively. In this section, we consider the case of composite knotcrystal - a composite system with four entangled vortex-membranes $(\mathbf{Z}(\vec{x}, t))$ and discuss the twist-writhe locking relation for it.

For the entanglement between $z_{\mathrm{A}_{1}, 1}(\vec{x}, t)$ and $z_{\mathrm{A}_{2}, 1}(\vec{x}, t)$ or that between $z_{\mathrm{B}_{1}, 1}(\vec{x}, t)$ and $z_{\mathrm{B}_{2}, 1}(\vec{x}, t)$, the twist-writhe locking relation is similar to that of the entanglement between $\mathrm{z}_{\mathrm{A}, 2}(\vec{x}, t)$ and $\mathrm{z}_{\mathrm{B}, 2}(\vec{x}, t)$ in a composite winding knot-crystal with $(\mathcal{N}=2, \mathcal{M}=2)$.

There are three types of vectors for topological numbers to describe entanglement between $z_{\mathrm{A}_{1}, 1}(\vec{x}, t)$ and $z_{\mathrm{A}_{2}, 1}(\vec{x}, t)$ :a $1 \mathrm{D}$ linking number density-vector 


$$
\begin{aligned}
\boldsymbol{\zeta}_{\left(\mathrm{A}_{1} \mathrm{~A}_{2}\right), 1 \mathrm{D}}= & \left(\zeta_{\left(\mathrm{A}_{1} \mathrm{~A}_{2}\right), 1 \mathrm{D}}^{x}, \zeta_{\left(\mathrm{A}_{1} \mathrm{~A}_{2}\right), 1 \mathrm{D}}^{y}, \zeta_{\left(\mathrm{A}_{1} \mathrm{~A}_{2}\right), 1 \mathrm{D}}^{z}\right)^{13} \text { where } \\
& \zeta_{\left(\mathrm{A}_{1} \mathrm{~A}_{2}\right), 1 \mathrm{D}}^{I}=\frac{1}{4 \pi} \oint_{C_{x^{I}, \mathrm{~A}_{1}}} \oint_{C_{x^{I}, \mathrm{~A}_{2}}} \frac{\left(\mathbf{s}_{\mathrm{A}_{1}}^{I}-\mathbf{s}_{\mathrm{A}_{2}}^{I}\right) \cdot d \mathbf{s}_{\mathrm{A}_{1}}^{I} \times d \mathbf{s}_{\mathrm{A}_{2}}^{I}}{\left|\mathbf{s}_{\mathrm{A}_{1}}^{I}-\mathbf{s}_{\mathrm{A}_{2}}^{I}\right|^{3}}
\end{aligned}
$$

a writhe number density vector

$$
\begin{aligned}
W_{\left(\mathrm{A}_{1} \mathrm{~A}_{2}\right), 1 \mathrm{D}}^{I}= & \frac{1}{4 \pi} \oint_{C_{x^{I}, \mathrm{~A}_{1}}} \oint_{C_{x^{I}, \mathrm{~A}_{1}}} \frac{\left(\mathbf{s}_{1}^{I}-\mathbf{s}_{2}\right) \cdot d \mathbf{s}_{1}^{I} \times d \mathbf{s}_{2}}{\left|\mathbf{s}_{1}^{I}-\mathbf{s}_{2}\right|^{3}} \\
& +\frac{1}{4 \pi} \oint_{C_{x^{I}, \mathrm{~A}_{2}}} \oint_{C_{x^{I}, \mathrm{~A}_{2}}} \frac{\left(\mathbf{s}_{1}^{I}-\mathbf{s}_{2}\right) \cdot d \mathbf{s}_{1}^{I} \times d \mathbf{s}_{2}}{\left|\mathbf{s}_{1}^{I}-\mathbf{s}_{2}\right|^{3}},
\end{aligned}
$$

a twist number density vector

$$
T_{\left(\mathrm{A}_{1} \mathrm{~A}_{2}\right), 1 \mathrm{D}}^{I}=\frac{1}{2 \pi} \oint_{C_{x^{I}, \mathrm{~A}_{1}}}\left(\mathbf{N}^{I} \times\left(\mathbf{N}^{I}\right)^{\prime}\right) \cdot d \mathbf{s}^{I}+\frac{1}{2 \pi} \oint_{C_{x^{I}, \mathrm{~A}_{2}}}\left(\mathbf{N}^{I} \times\left(\mathbf{N}^{I}\right)^{\prime}\right) \cdot d \mathbf{s}^{I} .
$$

There are three types of vectors for topological number to describe entanglement between $z_{\mathrm{B}_{1}, 1}(\vec{x}, t)$ and $z_{\mathrm{B}_{2}, 1}(\vec{x}, t)$ : a $1 \mathrm{D}$ linking number density-vector $\boldsymbol{\zeta}_{\left(\mathrm{B}_{1} \mathrm{~B}_{2}\right), 1 \mathrm{D}}=$ $\left(\zeta_{\left(\mathrm{B}_{1} \mathrm{~B}_{2}\right), 1 \mathrm{D}}^{x}, \zeta_{\left(\mathrm{B}_{1} \mathrm{~B}_{2}\right), 1 \mathrm{D}}^{y}, \zeta_{\left(\mathrm{B}_{1} \mathrm{~B}_{2}\right), 1 \mathrm{D}}^{z}\right),{ }^{13}$ where

$$
\zeta_{\left(\mathrm{B}_{1} \mathrm{~B}_{2}\right), 1 \mathrm{D}}^{I}=\frac{1}{4 \pi} \oint_{C_{x^{I}, \mathrm{~B}_{1}}} \oint_{C_{x^{I}, \mathrm{~B}_{2}}} \frac{\left(\mathbf{s}_{\mathrm{B}_{1}}^{I}-\mathbf{s}_{\mathrm{B}_{2}}^{I}\right) \cdot d \mathbf{s}_{\mathrm{B}_{1}}^{I} \times d \mathbf{s}_{\mathrm{B}_{2}}^{I}}{\left|\mathbf{s}_{\mathrm{B}_{1}}^{I}-\mathbf{s}_{\mathrm{B}_{2}}^{I}\right|^{3}},
$$

a writhe number density vector

$$
\begin{aligned}
W_{\left(\mathrm{B}_{1} \mathrm{~B}_{2}\right), 1 \mathrm{D}}^{I}= & \frac{1}{4 \pi} \oint_{C_{x^{I}, \mathrm{~B}_{1}}} \oint_{C_{x^{I}, \mathrm{~B}_{1}}} \frac{\left(\mathbf{s}_{1}^{I}-\mathbf{s}_{2}\right) \cdot d \mathbf{s}_{1}^{I} \times d \mathbf{s}_{2}}{\left|\mathbf{s}_{1}^{I}-\mathbf{s}_{2}\right|^{3}} \\
& +\frac{1}{4 \pi} \oint_{C_{x^{I}, \mathrm{~B}_{2}}} \oint_{C_{x^{I}, \mathrm{~B}_{2}}} \frac{\left(\mathbf{s}_{1}^{I}-\mathbf{s}_{2}\right) \cdot d \mathbf{s}_{1}^{I} \times d \mathbf{s}_{2}}{\left|\mathbf{s}_{1}^{I}-\mathbf{s}_{2}\right|^{3}},
\end{aligned}
$$

a twist number density vector

$$
T_{\left(\mathrm{B}_{1} \mathrm{~B}_{2}\right), 1 \mathrm{D}}^{I}=\frac{1}{2 \pi} \oint_{C_{x^{I}, \mathrm{~B}_{1}}}\left(\mathbf{N}^{I} \times\left(\mathbf{N}^{I}\right)^{\prime}\right) \cdot d \mathbf{s}^{I}+\frac{1}{2 \pi} \oint_{C_{x^{I}, \mathrm{~B}_{2}}}\left(\mathbf{N}^{I} \times\left(\mathbf{N}^{I}\right)^{\prime}\right) \cdot d \mathbf{s}^{I} .
$$

The twist-writhe locking condition for two entangled vortex-membranes $\mathbf{Z}_{1, \mathrm{~A}}^{\prime}(\vec{x}, t)=\left(\begin{array}{l}z_{\mathrm{A}_{1}, 1}(\vec{x}, t) \\ z_{\mathrm{A}_{2}, 1}(\vec{x}, t)\end{array}\right)$ is given by

$$
\zeta_{\left(\mathrm{A}_{1} \mathrm{~A}_{2}\right), 1 \mathrm{D}}^{I}=W_{\left(\mathrm{A}_{1} \mathrm{~A}_{2}\right), 1 \mathrm{D}}^{I}+T_{\left(\mathrm{A}_{1} \mathrm{~A}_{2}\right), 1 \mathrm{D}}^{I} \equiv \text { const. }, \quad(I=x, y, z)
$$

or

$$
\delta W_{\left(\mathrm{A}_{1} \mathrm{~A}_{2}\right), 1 \mathrm{D}}^{I} \equiv-\delta T_{\left(\mathrm{A}_{1} \mathrm{~A}_{2}\right), 1 \mathrm{D}}^{I}, \quad(I=x, y, z) .
$$

The twist-writhe locking condition for two entangled vortex-membranes $\mathbf{Z}_{\mathrm{B}, 1}^{\prime}(\vec{x}, t)=\left(\begin{array}{l}z_{\mathrm{B}_{1}, 1}(\vec{x}, t) \\ z_{\mathrm{B}_{2}, 1}(\vec{x}, t)\end{array}\right)$ is given by

$$
\zeta_{\left(\mathrm{B}_{1} \mathrm{~B}_{2}\right), 1 \mathrm{D}}^{I}=W_{\left(\mathrm{B}_{1} \mathrm{~B}_{2}\right), 1 \mathrm{D}}^{I}+T_{\left(\mathrm{B}_{1} \mathrm{~B}_{2}\right), 1 \mathrm{D}}^{I} \equiv \text { const. }, \quad(I=x, y, z)
$$

or

$$
\delta W_{\left(\mathrm{B}_{1} \mathrm{~B}_{2}\right), 1 \mathrm{D}}^{I} \equiv-\delta T_{\left(\mathrm{B}_{1} \mathrm{~B}_{2}\right), 1 \mathrm{D}}^{I}, \quad(I=x, y, z) .
$$




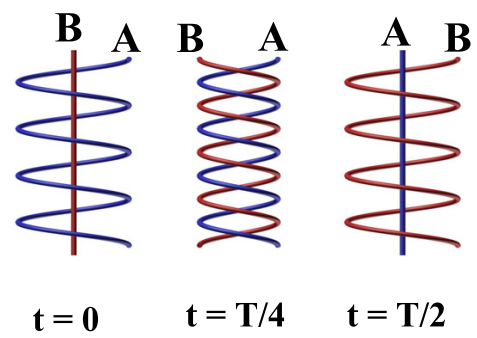

(a)

(b)

(c)

Fig. 5. (Color online) An illustration of leapfrogging motion for two entangled vortex-lines.

In particular, there exist the following topological locking relationships:

$$
W_{1 \mathrm{D}, \mathrm{A}}^{I} \Longrightarrow \delta W_{\mathrm{A}_{1}, 1 \mathrm{D}}^{I}=\delta W_{\mathrm{A}_{2}, 1 \mathrm{D}}^{I}=W_{1 \mathrm{D}, \mathrm{A}}^{I}
$$

and

$$
W_{1 \mathrm{D}, \mathrm{B}}^{I} \Longrightarrow \delta W_{\mathrm{B}_{1}, 1 \mathrm{D}}^{I}=\delta W_{\mathrm{B}_{2}, 1 \mathrm{D}}^{I}=W_{1 \mathrm{D}, \mathrm{B}}^{I} .
$$

Because $W_{1 \mathrm{D}, \mathrm{A}}^{I}$ or $W_{1 \mathrm{D}, \mathrm{B}}^{I}$ is time-dependent due to leapfrogging motion, $W_{\mathrm{A}_{1}, 1 \mathrm{D}}^{I}$, $W_{\mathrm{A}_{2}, 1 \mathrm{D}}^{I}, W_{\mathrm{B}_{1}, 1 \mathrm{D}}^{I}, W_{\mathrm{B}_{2}, 1 \mathrm{D}}^{I}$ change with time $t$. See the illustration in Fig. 5.

According to twist-writhe locking conditions, $\delta W_{\left(\mathrm{A}_{1} \mathrm{~A}_{2}\right), 1 \mathrm{D}}^{I} \equiv-\delta T_{\left(\mathrm{A}_{1} \mathrm{~A}_{2}\right), 1 \mathrm{D}}^{I}$ and $\delta W_{\left(\mathrm{B}_{1} \mathrm{~B}_{2}\right), 1 \mathrm{D}}^{I} \equiv-\delta T_{\left(\mathrm{B}_{1} \mathrm{~B}_{2}\right), 1 \mathrm{D}}^{I}$, we have

$$
\delta T_{\left(\mathrm{A}_{1} \mathrm{~A}_{2}\right), 1 \mathrm{D}}^{I}=-W_{1 \mathrm{D}, \mathrm{A}}^{I}, \quad(I=x, y, z)
$$

and

$$
\delta T_{\left(\mathrm{B}_{1} \mathrm{~B}_{2}\right), 1 \mathrm{D}}^{I}=-W_{1 \mathrm{D}, \mathrm{B}}^{I}, \quad(I=x, y, z) .
$$

From the above discussion, we point out that for uniformly entangled vortexmembranes when the two vortex-membranes have additional global winding, finite $\delta W_{(\mathrm{AB}), 1 \mathrm{D}}^{I}$ leads to finite $-\delta T_{(\mathrm{AB}), 1 \mathrm{D}}^{I}$ that is really an additional entanglement between two vortex-membranes and vice versa. That means a global winding of A-knot-crystal and B-knot-crystal leads to additional internal twisting between vortex-membranes $A_{1}, A_{2}$ and vortex-membranes $B_{1}, B_{2}$.

\subsubsection{3-level composite knot-crystal with $(\mathcal{N}=4, \mathcal{M}=3)$}

A 3-level composite knot-crystal with $(\mathcal{N}=4, \mathcal{M}=3)$ is an object of four entangled vortex-membranes $A_{1}, A_{2}$ and $B_{1}, B_{2}$. We firstly tangle two symmetric vortexmembranes $A_{1}$ and $A_{2}$ and get $A$-knot-crystal and tangle two symmetric vortexmembranes $\mathrm{B}_{1}$ and $\mathrm{B}_{2}$ and get B-knot-crystal. Then, we tangle A-knot-crystal and B-knot-crystal into a 2-level composite double-helix knot-crystal. Finally, we wind the 2-level composite double-helix knot-crystal and get a 3-level composite knotcrystal with $(\mathcal{N}=4, \mathcal{M}=3)$. See the illustration in Fig. 6(a). In Fig. 6(b), we show the hierarchy structure of a composite knot-crystal with $(\mathcal{N}=4, \mathcal{M}=3)$. 


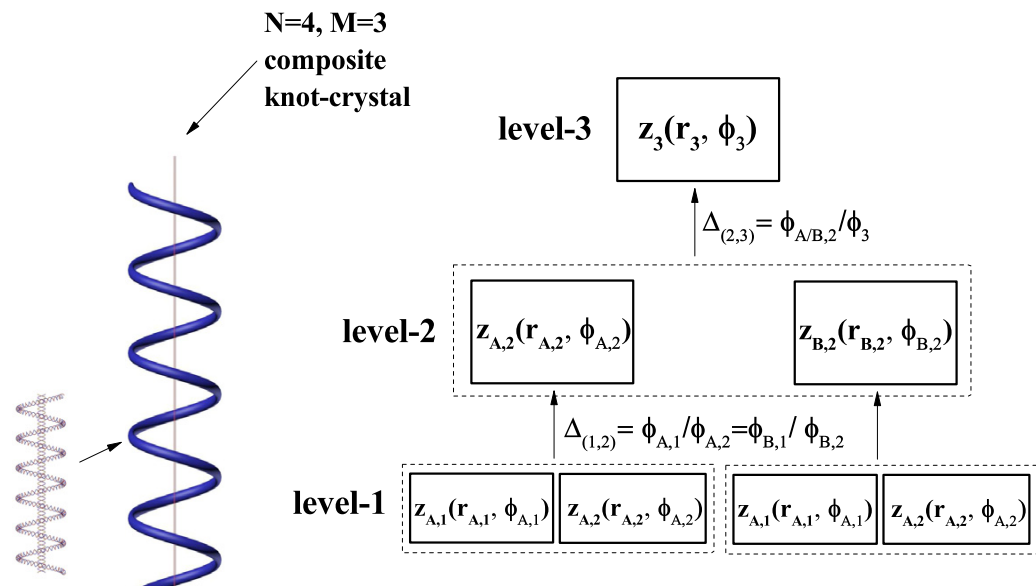

(a)

(b)

Fig. 6. (Color online) (a) An illustration of a 1D composite knot-crystal with $(\mathcal{N}=4$ and $\mathcal{M}=3)$ (a system of four entangled vortex-lines); (b) the hierarchy structure.

(a) The definition: In general, the function of 3-level composite knot-crystal with $(\mathcal{N}=4, \mathcal{M}=3)$ is denoted by

$$
\mathbf{Z}(\vec{x}, t)=\left(\begin{array}{lll}
\prod_{I} z_{\mathrm{A}_{1}, 1}\left(\phi_{\mathrm{A}_{1}, 1}^{I}, t\right) & \prod_{I} z_{\mathrm{A}_{1}, 2}\left(\phi_{\mathrm{A}_{1}, 2}^{I}, t\right) & \prod_{I} z_{\mathrm{A}_{1}, 3}\left(\phi_{\mathrm{A}_{1}, 3}^{I}, t\right) \\
\prod_{I} z_{\mathrm{A}_{2}, 1}\left(\phi_{\mathrm{A}_{2}, 1}^{I}, t\right) & \prod_{I} z_{\mathrm{A}_{2}, 2}\left(\phi_{\mathrm{A}_{2}, 2}^{I}, t\right) & \prod_{I} z_{\mathrm{A}_{2}, 3}\left(\phi_{\mathrm{A}_{2}, 3}^{I}, t\right) \\
\prod_{I} z_{\mathrm{B}_{1}, 1}\left(\phi_{\mathrm{B}_{1}, 1}^{I}, t\right) & \prod_{I} z_{\mathrm{B}_{1}, 2}\left(\phi_{\mathrm{B}_{1}, 2}^{I}, t\right) & \prod_{I} z_{\mathrm{B}_{1}, 3}\left(\phi_{\mathrm{B}_{1}, 3}^{I}, t\right) \\
\prod_{I} z_{\mathrm{B}_{2}, 1}\left(\phi_{\mathrm{B}_{2}, 1}^{I}, t\right) & \prod_{I} z_{\mathrm{B}_{2}, 2}\left(\phi_{\mathrm{B}_{2}, 2}^{I}, t\right) & \prod_{I} z_{\mathrm{B}_{2}, 3}\left(\phi_{\mathrm{B}_{2}, 3}^{I}, t\right)
\end{array}\right) .
$$

According to $\phi_{\mathrm{A}, 3}^{I}\left(x^{I}\right)=\phi_{\mathrm{B}, 3}^{I}\left(x^{I}\right)=\phi_{3}^{I}\left(x^{I}, t\right)$, or

$$
\begin{aligned}
\prod_{I} z_{\mathrm{A}_{1}, 3}\left(\phi_{\mathrm{A}_{1}, 3}^{I}, t\right) & =\prod_{I} z_{\mathrm{A}_{2}, 3}\left(\phi_{\mathrm{A}_{2}, 3}^{I}, t\right)=\prod_{I} z_{\mathrm{B}_{1}, 3}\left(\phi_{\mathrm{B}_{1}, 3}^{I}, t\right) \\
& =\prod_{I} z_{\mathrm{B}_{2}, 3}\left(\phi_{\mathrm{B}_{2}, 3}^{I}, t\right)=\mathrm{z}_{3}\left(x^{I}, t\right),
\end{aligned}
$$

$\mathrm{z}_{3}(\vec{x}, t)$ denotes the center membranes of A-knot-crystal and B-knot-crystal. The phase angles of $\mathrm{z}_{3}\left(x^{I}, t\right)$ are $\phi_{3}^{I}\left(x^{I}, t\right)$ and the winding radii of $\mathrm{z}_{3}(\vec{x}, t)$ are $r_{3}(\vec{x}, t)$. According to $\phi_{\mathrm{A}_{1}, 2}^{I}\left(x^{I}\right)=\phi_{\mathrm{A}_{2}, 2}^{I}\left(x^{I}\right)=\phi_{\mathrm{A}, 2}^{I}\left(x^{I}, t\right)$ and $\phi_{\mathrm{B}_{1}, 2}^{I}\left(x^{I}\right)=\phi_{\mathrm{B}_{2}, 2}^{I}\left(x^{I}\right)=$ $\phi_{\mathrm{B}, 2}^{I}\left(x^{I}, t\right)$ or

$$
\prod_{I} z_{\mathrm{A}_{1}, 2}\left(\phi_{\mathrm{A}_{1}, 2}^{I}\left(x^{I}\right), t\right)=\prod_{I} z_{\mathrm{A}_{2}, 2}\left(\phi_{\mathrm{A}_{2}, 2}^{I}\left(x^{I}\right), t\right)=\mathrm{z}_{\mathrm{A}, 2}(\vec{x}, t)
$$


and

$$
\prod_{I} z_{\mathrm{B}_{1}, 2}\left(\phi_{\mathrm{B}_{1}, 2}^{I}\left(x^{I}\right), t\right)=\prod_{I} z_{\mathrm{B}_{2}, 2}\left(\phi_{\mathrm{B}_{2}, 2}^{I}\left(x^{I}\right), t\right)=\mathrm{z}_{\mathrm{B}, 2}(\vec{x}, t)
$$

$\mathbf{Z}_{2}(\vec{x}, t)=\left(\begin{array}{l}\mathrm{z}_{\mathrm{A}, 2}(\vec{x}, t) \\ \mathrm{z}_{\mathrm{B}, 2}(\vec{x}, t)\end{array}\right)$ also denote the center membranes of A-knot-crystal and Bknot-crystal, respectively. $\mathrm{z}_{2, \mathrm{~A}}(\vec{x}, t)$ denotes the global position of the entangled vortex-membranes $\mathrm{A}_{1}, \mathrm{~A}_{2}$ and $\mathrm{z}_{\mathrm{B}, 2}(\vec{x}, t)$ denotes the global position of the entangled vortex-membranes $\mathrm{B}_{1}, \mathrm{~B}_{2}$.

$$
\begin{aligned}
& \mathbf{Z}_{\mathrm{A}, 1}^{\prime}(\vec{x}, t)=\left(\begin{array}{c}
z_{\mathrm{A}_{1}, 1}(\vec{x}, t) \\
z_{\mathrm{A}_{2}, 1}(\vec{x}, t)
\end{array}\right)=\left(\begin{array}{l}
\prod_{I}^{I} z_{\mathrm{A}_{1}, 1}\left(\phi_{\mathrm{A}_{1}, 1}^{I}\left(\phi_{\mathrm{A}_{1}, 2}^{I}\left(x^{I}\right)\right), t\right) \\
\prod_{I} z_{\mathrm{A}_{2}, 1}\left(\phi_{\mathrm{A}_{2}, 1}^{I}\left(\phi_{\mathrm{A}_{1}, 2}^{I}\left(x^{I}\right)\right), t\right)
\end{array}\right) \\
& \mathbf{Z}_{\mathrm{B}, 1}^{\prime}(\vec{x}, t)=\left(\begin{array}{c}
z_{\mathrm{B}_{1}, 1}(\vec{x}, t) \\
z_{\mathrm{B}_{2}, 1}(\vec{x}, t)
\end{array}\right)=\left(\begin{array}{l}
\prod_{I} z_{\mathrm{B}_{1}, 1}\left(\phi_{\mathrm{B}_{1}, 1}^{I}\left(\phi_{\mathrm{B}_{1}, 2}^{I}\left(x^{I}\right)\right), t\right) \\
\prod_{I} z_{\mathrm{B}_{2}, 1}\left(\phi_{\mathrm{B}_{2}, 1}^{I}\left(\phi_{\mathrm{B}_{1}, 2}^{I}\left(x^{I}\right)\right), t\right)
\end{array}\right)
\end{aligned}
$$

denote local entanglement between two vortex-membranes $\mathrm{A}_{1}, \mathrm{~A}_{2}$ and $\mathrm{B}_{1}, \mathrm{~B}_{2}$, respectively. The phase angles of $\mathrm{z}_{\mathrm{A} / \mathrm{B}, 2}\left(x^{I}, t\right)$ are $\phi_{\mathrm{A} / \mathrm{B}, 2}^{I}\left(x^{I}, t\right)$ and the winding radii of $\mathrm{z}_{\mathrm{A} / \mathrm{B}, 2}(\vec{x}, t)$ are $r_{\mathrm{A} / \mathrm{B}, 2}(\vec{x}, t)$. The winding radii $r_{\mathrm{A}_{i} / \mathrm{B}_{i}, 1}$ are the local winding radii of vortex-membranes $\mathbf{Z}_{\mathrm{A}_{i} / \mathrm{B}_{i}, 1}^{\prime}(\vec{x}, t)$ around its center; $\phi_{\mathrm{A}_{i} / \mathrm{B}_{i}, 1}^{I}\left(x^{I}, t\right)$ are the angles of vortex-membranes $\mathbf{Z}_{\mathrm{A}_{i} / \mathrm{B}_{i}, 1}^{\prime}(\vec{x}, t)$ around its center membrane A/B.

In particular, we consider a perturbative condition,

$$
r_{3} \gg r_{2}=\sqrt{r_{\mathrm{A}, 2}^{2}(\vec{x}, t)+r_{\mathrm{B}, 2}^{2}(\vec{x}, t)} \gg\left|r_{\mathrm{A}_{i} / \mathrm{B}_{i}, 1}(\vec{x}, t)\right| .
$$

The hierarchy series is

$$
\left\{\Delta_{(1,2)}, \Delta_{(2,3)}\right\}
$$

where

$$
\Delta_{(1,2)}=\frac{\phi_{\mathrm{A}_{1}, 1}^{I}\left(x^{I}, t\right)}{\phi_{\mathrm{A}, 2}^{I}\left(x^{I}, t\right)}=\frac{\phi_{\mathrm{B}_{1}, 1}^{I}\left(x^{I}, t\right)}{\phi_{\mathrm{B}, 2}^{I}\left(x^{I}, t\right)}=\frac{\phi_{\mathrm{A}_{2}, 1}^{I}\left(x^{I}, t\right)}{\phi_{\mathrm{A}, 2}^{I}\left(x^{I}, t\right)}=\frac{\phi_{\mathrm{B}_{2}, 1}^{I}\left(x^{I}, t\right)}{\phi_{\mathrm{B}, 2}^{I}\left(x^{I}, t\right)}
$$

and

$$
\Delta_{(2,3)}=\frac{\phi_{\mathrm{A}, 2}^{I}\left(x^{I}, t\right)}{\phi_{3}^{I}\left(x^{I}, t\right)}=\frac{\phi_{\mathrm{B}, 2}^{I}\left(x^{I}, t\right)}{\phi_{3}^{I}\left(x^{I}, t\right)} .
$$

(b) Example: In this paper, we focus on a particular type of 3-level composite knot-crystal with $(\mathcal{N}=4, \mathcal{M}=3)$.

$\mathrm{z}_{3}(\vec{x}, t)$ is $\sigma_{z}$-type Kelvin wave. The spin network state is

$$
\begin{aligned}
& \left\langle\sigma^{X} \otimes \overrightarrow{1}\right\rangle=\vec{n}_{\sigma}^{X}=(1,0,0), \\
& \left\langle\sigma^{Y} \otimes \overrightarrow{1}\right\rangle=\vec{n}_{\sigma}^{Y}=(1,0,0), \\
& \left\langle\sigma^{Z} \otimes \overrightarrow{1}\right\rangle=\vec{n}_{\sigma}^{Z}=(1,0,0) .
\end{aligned}
$$


$\mathbf{Z}_{2}(\vec{x}, t)$ becomes an SOC knot-crystal, i.e.,

$$
\mathbf{Z}_{2}(\vec{x}, t)=\left(\begin{array}{l}
\mathrm{z}_{2, \mathrm{~A}}(\vec{x}, t) \\
\mathrm{z}_{2, \mathrm{~B}}(\vec{x}, t)
\end{array}\right) .
$$

The tensor network states of $\mathbf{Z}_{2}(\vec{x}, t)$ are

$$
\begin{aligned}
& \left\langle\sigma^{X} \otimes \overrightarrow{1}\right\rangle=\vec{n}_{\sigma}^{X}=(1,0,0), \\
& \left\langle\sigma^{Y} \otimes \overrightarrow{1}\right\rangle=\vec{n}_{\sigma}^{Y}=(0,1,0), \\
& \left\langle\sigma^{Z} \otimes \overrightarrow{1}\right\rangle=\vec{n}_{\sigma}^{Z}=(0,0,1) .
\end{aligned}
$$

$\mathbf{Z}_{\mathrm{A}, 1}^{\prime}(\vec{x}, t)$ and $\mathbf{Z}_{\mathrm{B}, 1}^{\prime}(\vec{x}, t)$ are SOC knot-crystal with leapfrogging motion. The tensor states are also

$$
\begin{aligned}
& \left\langle\sigma^{X} \otimes \overrightarrow{1}\right\rangle=\vec{n}_{\sigma}^{X}=(1,0,0), \\
& \left\langle\sigma^{Y} \otimes \overrightarrow{1}\right\rangle=\vec{n}_{\sigma}^{Y}=(1,0,0), \\
& \left\langle\sigma^{Z} \otimes \overrightarrow{1}\right\rangle=\vec{n}_{\sigma}^{Z}=(1,0,0) .
\end{aligned}
$$

The hierarchy number between level-1 and level- $2 \Delta_{(1,2)}$ is considered to be a positive integer number $n$ (for example, $n=3$ ).

For the 3-level composite knot-crystal with $(\mathcal{N}=4, \mathcal{M}=3)$, there exists complex twist-writhe locking condition. One can use the above approach to discuss the twist-writhe locking condition for a 3-level composite knot-crystal with $(\mathcal{N}=4$, $\mathcal{M}=3)$.

\section{Zero-Lattice and Zeroes}

\subsection{Projection of vortex-membranes}

There are two types of projections on vortex-membranes: the projection for single vortex-membrane and that for entangled vortex-membranes. We call the projection for single vortex-membrane $W$-type projection and that for entangled vortexmembranes T-type projection. See the illustration in Fig. 7.

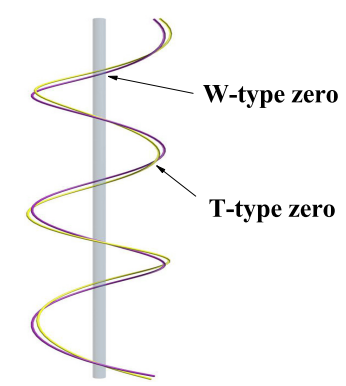

Fig. 7. (Color online) An illustration of a $W$-type zero from $W$-type projection and a $T$-type zero from $T$-type projection. 


\subsubsection{Projection for single vortex-membrane}

Firstly, we discuss the projection for single vortex-membrane of which the function is

$$
\mathrm{z}(\vec{x}, t)=\xi(\vec{x}, t)+i \eta(\vec{x}, t) .
$$

To locally characterize the windings of a helical vortex-membrane, we define the projection via a projection angle $\theta$ on $\{\xi(\vec{x}, t), \eta(\vec{x}, t)\}$ by $\hat{P}_{\theta}\left(\begin{array}{l}\xi(\vec{x}, t) \\ \eta(\vec{x}, t)\end{array}\right)=\left(\begin{array}{c}\xi_{\theta}(\vec{x}, t) \\ {\left[\eta_{\theta}(\vec{x}, t)\right]_{0}}\end{array}\right)$, where $\xi_{\theta}(\vec{x}, t)=\xi(\vec{x}, t) \cos \theta-\eta(\vec{x}, t) \sin \theta$ is variable and $\left[\eta_{\theta}(\vec{x}, t)\right]_{0}=\xi(\vec{x}, t) \sin \theta+$ $\eta(\vec{x}, t) \cos \theta$ is constant. Thus, the projected helical vortex-membrane is described by the function $\xi(\vec{x}, t)$. A crossing between a helical vortex-membrane and a straight one $(z(\vec{x}, t)=0)$ in its center corresponds to a solution of the equation

$$
\hat{P}_{\theta}[\mathrm{z}(\vec{x}, t)]=0,
$$

that is $\xi_{\theta}(\vec{x}, t)=0$. We call the equation a zero equation and its solution a zero solution.

\subsubsection{Projection for entangled vortex-membranes}

Next, we discuss the projection for entangled vortex-membranes. For two entangled vortex-membranes described by $\mathrm{z}_{\mathrm{A} / \mathrm{B}}(\vec{x}, t)=\xi_{\mathrm{A} / \mathrm{B}}(\vec{x}, t)+i \eta_{\mathrm{A} / \mathrm{B}}(\vec{x}, t)$, the projection along a given direction $\theta$ in $5 \mathrm{D}$ space is defined by

$$
\hat{P}_{\theta}\left(\begin{array}{c}
\xi_{\mathrm{A} / \mathrm{B}}(\vec{x}, t) \\
\eta_{\mathrm{A} / \mathrm{B}}(\vec{x}, t)
\end{array}\right)=\left(\begin{array}{c}
\xi_{\mathrm{A} / \mathrm{B}, \theta}(\vec{x}, t) \\
{\left[\eta_{\mathrm{A} / \mathrm{B}, \theta}(\vec{x}, t)\right]_{0}}
\end{array}\right),
$$

where $\xi_{\mathrm{A} / \mathrm{B}, \theta}(\vec{x}, t)=\xi_{\mathrm{A} / \mathrm{B}}(\vec{x}, t) \cos \theta-\eta_{\mathrm{A} / \mathrm{B}}(\vec{x}, t) \sin \theta$ is variable and $\left[\eta_{\mathrm{A} / \mathrm{B}, \theta}\right.$ $(\vec{x}, t)]_{0}=\xi_{\mathrm{A} / \mathrm{B}}(\vec{x}, t) \sin \theta+\eta_{\mathrm{A} / \mathrm{B}}(\vec{x}, t) \cos \theta$ is constant. So, the projected vortexmembrane is described by the function $\xi_{\mathrm{A} / \mathrm{B}, \theta}(\vec{x}, t)$. For two projected vortexmembranes described by $\xi_{\mathrm{A}, \theta}(\vec{x}, t)$ and $\xi_{\mathrm{B}, \theta}(\vec{x}, t)$, a zero is the solution of the equation

$$
\hat{P}_{\theta}\left[\mathrm{z}_{\mathrm{A}}(\vec{x}, t)\right] \equiv \xi_{\mathrm{A}, \theta}(\vec{x}, t)=\hat{P}_{\theta}\left[\mathrm{z}_{\mathrm{B}}(\vec{x}, t)\right] \equiv \xi_{\mathrm{B}, \theta}(\vec{x}, t)
$$

\subsection{Zero-lattice}

We then introduce two types of zero-lattices by the two types of projections.

\subsubsection{W-type zero-lattice from $W$-type projection on a helical vortex-membrane}

Firstly, we consider the zero-lattice from $W$-type projection on a helical vortexmembrane.

The function of a $1 \mathrm{D}$ helical vortex-line in a $3 \mathrm{D}$ fluid is

$$
\mathrm{z}(x, t)=\xi(x, t)+i \eta(x, t)=r_{0} e^{ \pm i k_{0} \cdot x-i \omega_{0} t+i \phi_{0}},
$$

where $r_{0}$ is the winding radius of vortex-line that is set as constant, $k_{0}=\frac{\pi}{a}>0$ and $a$ is a fixed length that denotes the half pitchof the windings. $\phi_{0}$ is a constant angle. 


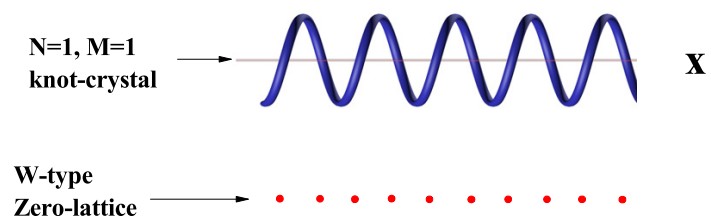

(a)

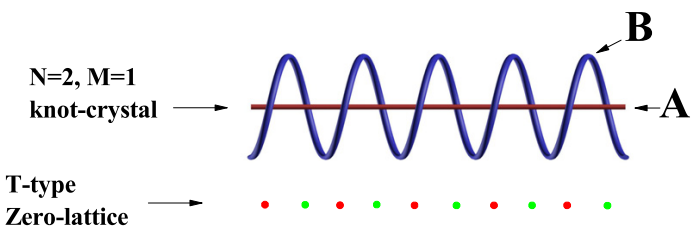

(b)

Fig. 8. (Color online) (a) An illustration of a $W$-type zero-lattice, (b) an illustration of a $T$-type zero-lattice.

\pm denotes two possible chiralities: left-hand with clockwise winding, or right-hand with counterclockwise winding.

For a helical vortex-line, from the zero solution $\xi(x, t)=0$, we get the zero solutions to be $\pm \bar{x}(t)=a \cdot X+\frac{a}{\pi} \omega_{0} t$, where $X$ is an integer along $x$-direction and $\theta=-\frac{\pi}{2}+\phi_{0}$. From the projection of a helical vortex-membrane, we have a crystal of crossings. See the illustration in Fig. 8(a) (the red dots denote the crossings that form a 1D lattice). Because the winding number of helical vortex-line is half of the crossing number, each crossing corresponds to a piece of helical vortex-line with half winding number. We call the object with half winding number a knot. As a result, the system can be regarded as a crystal of knots. It is obvious that the global rotation does not change the winding number density.

For an arbitrary 1D Kelvin wave with different spin states, the zero solution does not change i.e., $\left\langle\sigma^{Z}\right\rangle=\vec{n}_{\sigma}^{Z}=(0,0,1) \rightarrow \vec{n}_{\sigma}=\left(n_{x}, n_{y}, n_{x}\right)$ with $\left|\vec{n}_{\sigma}\right|=1$. 13

Therefore, in the following parts, we call the crystal with discrete lattice sites described by the integer numbers $X$ as "zero-lattice". 17

For a 3D SOC Kelvin wave of single vortex-membrane, we can use similar $W$ type projection to obtain a $3 \mathrm{D} W$-type zero-lattice.

\subsubsection{T-type zero-lattice from T-type projection on two entangled vortex-membranes}

For two entangled vortex-membranes, there exists leapfrogging motion. So, we call it leapfrogging knot-crystal. A leapfrogging knot-crystal (two entangled vortexmembranes) is described by

$$
\mathbf{Z}(\vec{x}, t)=\left(\begin{array}{c}
\mathrm{z}_{\mathrm{A}}(\vec{x}, t) \\
\mathrm{z}_{\mathrm{B}}(\vec{x}, t)
\end{array}\right)=\left(\begin{array}{l}
r_{\mathrm{A}} \\
r_{\mathrm{B}}
\end{array}\right) e^{i \vec{k} \cdot \vec{x}-i \omega_{0} t+i \omega^{*} t / 2},
$$


where $r_{\mathrm{A}}=r_{0} \cos \left(\frac{\omega^{*} t}{2}\right)$ and $r_{\mathrm{B}}=-r_{0} i \sin \left(\frac{\omega^{*} t}{2}\right)$. According to the knot equation $\hat{P}_{\theta}\left[z_{\mathrm{A}}(\vec{x})\right]=\hat{P}_{\theta}\left[z_{\mathrm{B}}(\vec{x})\right]$, we have

$$
\bar{x}_{\mathrm{F}, 0}^{I}=a \cdot X^{I}+\frac{a}{\pi} \omega_{0} t,
$$

where $\theta=-\frac{\pi}{2}$ and $\bar{x}_{\mathrm{F}, 0}^{I}=\vec{x} \cdot \vec{e}^{I}$ is the coordination on the axis along a given direction $\vec{e}^{I}$ and $X^{I}$ is an integer number. As a result, we also have a periodic distribution of zeroes (knots) that is a $T$-type zero-lattice. See the illustration in Fig. 8(b).

\subsubsection{Generalized spatial translation symmetry for zero-lattices}

For both types of zero-lattice, owing to the generalized spatial translation symmetry for the vortex-membranes, there exist corresponding generalized spatial translation symmetries. For example, for a helical vortex-membrane, by doing a spatial translation operation $\mathcal{T}(\Delta x)=e^{i \Delta x \cdot \hat{k}^{I}}$, we have

$$
\mathrm{z}(\vec{x}, t) \longrightarrow \mathrm{z}(\vec{x}+\Delta \vec{x}, t)=e^{ \pm i(\vec{k} \cdot \Delta \vec{x})} \mathrm{z}(\vec{x}, t) .
$$

Under the spatial transformation, the zero-lattices shift, i.e.,

$$
\bar{x}^{I}(t) \rightarrow\left(\bar{x}^{I}(t)\right)^{\prime}=\bar{x}^{I}(t)+\Delta x^{I} .
$$

However, after changing the projection angle, $\theta \rightarrow \theta+\frac{\pi}{a} \Delta x^{I}$, the zero-lattice is invariant,

$$
\bar{x}^{I}(t) \rightarrow\left(\bar{x}^{I}(t)\right)^{\prime}=\bar{x}^{I}(t) .
$$

Therefore, for the zero-lattices, the generalized spatial translation operation is also a combination of a continuum spatial translation operation and a global phase rotation operation.

\subsection{Zeroes and knots}

From the point of view of "information", each zero becomes the element of a zerolattice. Thus, the information of vortex-membranes is characterized by the distribution of zeroes. For the case of an extra zero, we have a knot; for the case of missing zero, we have an anti-knot. According to the existence of two types of zero-lattices, there are two types of zeroes: $W$-type zero and $T$-type zero. Obviously, a $W$-type zero is an element object of $W$-type zero-lattice and a $T$-type zero is an element object of $T$-type zero-lattice.

\subsection{1. $W$-type zero and $W$-type knot}

The element of $W$-type zero-lattice is $W$-type zero that corresponds to a crossing of a helical vortex-membrane and a straight line in its center. In the following parts, we call a knot with half winding number corresponding to $W$-type zero $W$-type knot. 
From the point of view of information, a knot is an information unit with fixed geometric properties that is always anti-phase-changing along arbitrary direction $\vec{e}$. When there exists a knot, the periodic boundary condition of Kelvin waves along arbitrary direction is changed into an anti-periodic boundary condition. Based on the projected vortex-membranes, we define a knot by a monotonic function $F_{\theta}(x)=$ $\xi_{\theta}(x)$ with

$$
\operatorname{sgn}\left[F_{\theta}(x \rightarrow-\infty) \cdot F_{\theta}(x \rightarrow \infty)\right]=-1,
$$

where $x$ denotes the position along the given direction $\vec{e}$. So, the sign-switching character can be labeled by winding number $w_{1 \mathrm{D}}$. The winding number $w_{1 \mathrm{D}}$ for a knot along the given direction is $\pm \frac{1}{2}$. On the other hand, from the topological character of a knot, there must exist a point, each knot corresponds to a zero along the given direction.

The inset in Fig. 9(a) is an illustration of a 1D unified $W$-type knot. Its function is given by

$$
\mathrm{z}(x)=r_{0} e^{i \phi(x)}
$$

where

$$
\phi(x)=\left\{\begin{array}{ll}
\phi_{0} \mp \frac{\pi}{2}, & x \in\left(-\infty, x_{0}\right], \\
\phi_{0} \mp \frac{\pi}{2} \pm k_{0}\left(x-x_{0}\right), & x \in\left(x_{0}, x_{0}+a\right], \\
\phi_{0} \pm \frac{\pi}{2}, & x \in\left(x_{0}+a, \infty\right),
\end{array}\right\}
$$

where $+\left( \pm k_{0}\left(x-x_{0}\right)\right)$ denotes a clockwise winding and - denotes a counterclockwise winding. There is a linear relationship between $\phi(x)$ and $x$ as $\phi(x) \propto x-x_{0}$ in the winding region of $x_{0}<x \leq x_{0}+a$. Thus, we obtain an anti-periodic boundary condition for the system,

$$
\phi(x \rightarrow \infty)-\phi(x \rightarrow-\infty)= \pm \pi .
$$

Under projection, we have the knot equation as

$$
x-x_{0}=\left( \pm \frac{\pi}{2} \pm \frac{\pi}{2}+\theta-\phi_{0}\right) / k_{0}
$$

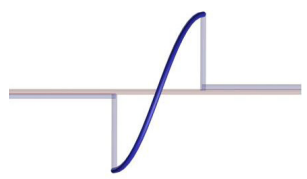

W-type knot

(a)

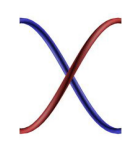

T-type knot

(b)

Fig. 9. (Color online) (a) A illustration of a $W$-type knot; (b) an illustration of a $T$-type knot. 


\subsubsection{T-type zero and T-type knot}

The element of the projected two entangled vortex-membranes is $T$-type zero that corresponds to a crossing of the two projected vortex-membranes. In the following parts, we call a knot with half twisting number corresponding to $T$-type zero $T$-type knot.

Based on the projected vortex-membranes, we define a knot by a monotonic function $F_{\theta}(x)=\xi_{\mathrm{A}, \theta}(x)-\xi_{\mathrm{B}, \theta}(x)$ with

$$
\operatorname{sgn}\left[F_{\theta}(x \rightarrow-\infty) \cdot F_{\theta}(x \rightarrow \infty)\right]=-1,
$$

where $x$ denotes the position along the given direction $\vec{e}$. From the topological character of a knot, each knot corresponds to a zero between two vortex-membranes along the given direction. See the illustration in Fig. 9(b).

A knot (a zero) has four degrees of freedom: two spin degrees of freedom $\uparrow$ or $\downarrow$ from the helicity degrees of freedom, the other two from the vortex degrees of freedom that characterize the vortex-membranes, A or B. For example, for an up-spin knot on vortex-membrane-A, the function is defined by $\mathrm{z}_{\mathrm{knot}, \uparrow, \mathrm{A}}(x, t)=$ $r_{\uparrow, \mathrm{A}} \exp \left[i \phi_{\mathrm{knot}, \uparrow, \mathrm{A}}(x, t)\right]$ where

$$
\phi_{\mathrm{knot}, \uparrow, \mathrm{A}}(x)=\left\{\begin{array}{ll}
-\phi_{0}+\frac{\pi}{2}, & x \in\left(-\infty, x_{0}\right] \\
-\phi_{0}+\frac{\pi}{2}+k_{0}\left(x-x_{0}\right), & x \in\left(x_{0}, x_{0}+a\right] \\
-\phi_{0}-\frac{\pi}{2}, & x \in\left(x_{0}+a, \infty\right)
\end{array}\right\}
$$

where $x=\vec{x} \cdot \vec{e}$ is the coordination on the axis along a given direction $\vec{e}$ and $\phi_{0}$ is an arbitrary constant angle, $k_{0}=\frac{\pi}{a}$.

\subsection{Examples}

\subsubsection{2-level composite knot-crystal with $(\mathcal{N}=2, \mathcal{M}=2)$}

For 2-level composite knot-crystal with $(\mathcal{N}=2, \mathcal{M}=2)$, the function of the center membranes of vortex-membrane-A and vortex-membrane-B $\mathrm{z}_{2}(\vec{x}, t)$; the function of the vortex-membrane- $\mathrm{A}$ is $\mathrm{z}_{\mathrm{A}, 1}(\vec{x}, t)$ and the function of the vortex-membrane- $\mathrm{B}$ is $\mathrm{z}_{\mathrm{B}, 1}(\vec{x}, t)$.

The level-2 $W$-type zero-lattice of the composite winding knot-crystal with $(\mathcal{N}=$ $2, \mathcal{M}=2$ ) is obtained by level- $2 W$-type projection of center membranes of vortexmembrane-A and vortex-membrane-B, i.e.,

$$
\hat{P}_{\theta_{2}}\left[\mathrm{z}_{2}(\vec{x}, t)\right]=0,
$$

that is $\xi_{2}(\vec{x}, t)=0$. The solution of zero-lattice is given by $\bar{x}^{I}(t)=a_{2} \cdot X^{I}+\frac{a_{2}}{\pi} \omega_{0} t$ where $X^{I}$ is an integer along $x^{I}$-direction and $\theta_{2}=-\frac{\pi}{2}+\phi_{2,0} . a_{2}$ is a fixed length that denotes the half pitch of the windings of $\mathrm{z}_{2}(\vec{x}, t)$. 
The level-1 $T$-type zero-lattice of the 2-level composite knot-crystal with $(\mathcal{N}=$ $2, \mathcal{M}=2$ ) is obtained by level-1 $T$-type projection of vortex-membrane-A and vortex-membrane-B, i.e.,

$$
\hat{P}_{\theta_{1}}\left[\mathrm{z}_{\mathrm{A}, 1}(\vec{x}, t)\right]=\hat{P}_{\theta_{2}}\left[\mathrm{z}_{\mathrm{B}, 1}(\vec{x}, t)\right]
$$

that is $\xi_{\mathrm{A}, 1}(\vec{x}, t)=\xi_{\mathrm{B}, 1}(\vec{x}, t)$.

In particular, there exists an intrinsic relationship between the number of $T$-type zeroes and level-2 $W$-type zeroes,

$$
\zeta_{(\mathrm{A}, \mathrm{B}), 1 \mathrm{D}}^{I}=W_{(\mathrm{A}, \mathrm{B}), 1 \mathrm{D}}^{I}+T_{(\mathrm{A}, \mathrm{B}), 1 \mathrm{D}}^{I} \equiv \text { const. }, \quad(I=x, y, z),
$$

where $\zeta_{(\mathrm{A}, \mathrm{B}), 1 \mathrm{D}}^{I}, W_{(\mathrm{A}, \mathrm{B}), 1 \mathrm{D}}^{I}$, and $T_{(\mathrm{A}, \mathrm{B}), 1 \mathrm{D}}^{I}$ are linking number, writhe number and twist number, respectively. Here, the number of level-1 $T$-type zeroes is equal to the twist number $T_{(\mathrm{A}, \mathrm{B}), 1 \mathrm{D}}^{I}$ and the number of level-2 $W$-type zeroes is equal to be the writhe number $W_{(\mathrm{A}, \mathrm{B}), 1 \mathrm{D}}^{I}$, respectively. Because the sum of the number of level-2 $T$-type zeroes and level-1 $W$-type zeroes is invariant, when one winds two entangled vortex-membranes (a 1-level knot-crystal with $(\mathcal{N}=2, \mathcal{M}=1)$ ) into a 2-level composite knot-crystal with $(\mathcal{N}=2, \mathcal{M}=2)$, some level-1 $T$-type of zeroes are replaced by level-2 $W$-type zeroes. We call it substitution effect of level-1 T-type of zeroes by level-2 $W$-type zeroes.

\subsubsection{2-level composite knot-crystal with $(\mathcal{N}=4, \mathcal{M}=2)$}

For 2-level composite knot-crystal with $(\mathcal{N}=4, \mathcal{M}=2)$, the functions $\mathbf{Z}_{2}(\vec{x}, t)=$ $\left(\begin{array}{l}\mathrm{z}_{\mathrm{A}, 2}(\vec{x}, t) \\ \mathrm{z}_{\mathrm{B}, 2}(\vec{x}, t)\end{array}\right)$ are described by another knot-crystal that characterizes the center membrane of A-knot-crystal by $\mathrm{z}_{\mathrm{A}, 2}(\vec{x}, t)$ and that of B-knot-crystal by $\mathrm{z}_{\mathrm{B}, 2}(\vec{x}, t)$, respectively. The A-knot-crystal (the entangled vortex-membranes $A_{1}, A_{2}$ ) around the $\mathrm{z}_{\mathrm{A}, 2}(\vec{x}, t)$ is described by

$$
\mathbf{Z}_{\mathrm{A}, 1}^{\prime}(\vec{x}, t)=\left(\begin{array}{l}
z_{\mathrm{A}_{1}, 1}(\vec{x}, t) \\
z_{\mathrm{A}_{2}, 1}(\vec{x}, t)
\end{array}\right)=\left(\begin{array}{l}
\prod_{I} z_{\mathrm{A}_{1}, 1}\left(\phi_{\mathrm{A}_{1}, 1}^{I}\left(\phi_{\mathrm{A}_{1}, 2}^{I}\left(x^{I}\right)\right), t\right) \\
\prod_{I} z_{\mathrm{A}_{2}, 1}\left(\phi_{\mathrm{A}_{2}, 1}^{I}\left(\phi_{\mathrm{A}_{1}, 2}^{I}\left(x^{I}\right)\right), t\right)
\end{array}\right)
$$

and B-knot-crystal (the entangled vortex-membranes $\mathrm{B}_{1}, \mathrm{~B}_{2}$ ) around $\mathrm{z}_{\mathrm{B}, 2}(\vec{x}, t)$ is described by

$$
\mathbf{Z}_{\mathrm{B}, 1}^{\prime}(\vec{x}, t)=\left(\begin{array}{l}
z_{\mathrm{B}_{1}, 1}(\vec{x}, t) \\
z_{\mathrm{B}_{2}, 1}(\vec{x}, t)
\end{array}\right)=\left(\begin{array}{l}
\prod_{I} z_{\mathrm{B}_{1}, 1}\left(\phi_{\mathrm{B}_{1}, 1}^{I}\left(\phi_{\mathrm{B}_{1}, 2}^{I}\left(x^{I}\right)\right), t\right) \\
\prod_{I} z_{\mathrm{B}_{2}, 1}\left(\phi_{\mathrm{B}_{2}, 1}^{I}\left(\phi_{\mathrm{B}_{1}, 2}^{I}\left(x^{I}\right)\right), t\right)
\end{array}\right) .
$$

After projection, there are five zero-lattices: level- $W$-type zero-lattice for Aknot-crystal, level-2 $W$-type zero-lattice for B-knot-crystal, level-2 $T$-type zerolattice between A-knot-crystal and B-knot-crystal, level-1 $T$-type zero-lattice between two entangled vortex-membranes $A_{1}$ and $A_{2}$ for $\mathrm{A}$-knot-crystal, level-1 
T-type zero-lattice between two entangled vortex-membranes $\mathrm{B}_{1}$ and $\mathrm{B}_{2}$ for B-knotcrystal.

The level-2 $W$-type zero-lattice for A-knot-crystal is obtained by level-2 $W$-type projection of vortex-membrane-A, i.e.,

$$
\hat{P}_{\theta_{2}}\left[\mathrm{z}_{\mathrm{A}, 2}(\vec{x}, t)\right]=0,
$$

that is $\xi_{\mathrm{A}, 2}(\vec{x}, t)=0$. The solution of the zero-lattice is obtained as

$$
\bar{x}_{2 W, \mathrm{~A}, 0}^{I}=a_{2} \cdot X^{I}+\frac{n a_{2}}{\pi} \omega_{0} t+\phi_{2 W, \mathrm{~A}, 0},
$$

where $\theta_{2}=-\frac{\pi}{2}$ and $X^{I}$ is an integer number along $x^{I}$-direction. $\phi_{2 W, \mathrm{~A}, 0}$ is a constant phase angle and $a_{2}$ is a fixed length that denotes the half pitch of the half windings of $\mathrm{z}_{\mathrm{A}, 2}(\vec{x}, t)$.

The level-2 $W$-type zero-lattice for B-knot-crystal is obtained by level- $2 W$-type projection of vortex-membrane-B, i.e.,

$$
\hat{P}_{\theta_{2}}\left[\mathrm{Z}_{\mathrm{B}, 2}(\vec{x}, t)\right]=0,
$$

that is $\xi_{\mathrm{B}, 2}(\vec{x}, t)=0$. The solution of the zero-lattice is obtained as

$$
\bar{x}_{2 W, \mathrm{~B}, 0}^{I}=a_{2} \cdot X^{I}+\frac{n a_{2}}{\pi} \omega_{0} t+\phi_{2 W, \mathrm{~B}, 0},
$$

where $\theta_{2}=-\frac{\pi}{2}$ and $X^{I}$ is an integer number along $x^{I}$-direction. $\phi_{2 W, \mathrm{~B}, 0}$ is a constant phase angle and $a_{2}$ is a fixed length that denotes the half pitch of the windings of $\mathrm{z}_{\mathrm{B}, 2}(\vec{x}, t)$.

The level-2 $T$-type zero-lattice is obtained by level-2 $T$-type projection between A-knot-crystal and B-knot-crystal, i.e.,

$$
\hat{P}_{\theta_{2}}\left[\mathrm{z}_{\mathrm{A}, 2}(\vec{x}, t)\right]=\hat{P}_{\theta}\left[\mathrm{z}_{\mathrm{B}, 2}(\vec{x}, t)\right],
$$

that is $\xi_{\mathrm{A}, 2}(\vec{x}, t)=\xi_{\mathrm{B}, 2}(\vec{x}, t)$. The solution of the zero-lattice is obtained as

$$
\bar{x}_{2 T, 0}^{I}=a_{2} \cdot X^{I}+\frac{n a_{2}}{\pi} \omega_{0} t+\phi_{2 T, 0}
$$

where $\theta_{2}=-\frac{\pi}{2}$ and $X^{I}$ is an integer number along $x^{I}$-direction. $\phi_{2 T, 0}$ is a constant phase angle and $a_{2}$ is a fixed length that denotes the half pitch of the twistings of $\mathrm{z}_{\mathrm{A} / \mathrm{B}, 2}(\vec{x}, t)$.

The level-1 $T$-type zero-lattice between two entangled vortex-membranes $\mathrm{A}_{1}$ and $\mathrm{A}_{2}$ for A-knot-crystal is obtained by level-1 T-type projection of A-knot-crystal,

$$
\hat{P}_{\theta_{1}}\left[z_{\mathrm{A}_{1}, 1}(\vec{x}, t)\right]=\hat{P}_{\theta}\left[z_{\mathrm{A}_{2}, 1}(\vec{x}, t)\right],
$$

i.e., $\xi_{\mathrm{A}_{1}, 1}(\vec{x}, t)=\xi_{\mathrm{A}_{2}, 1}(\vec{x}, t)$. The solution of the zero-lattice is obtained as

$$
\bar{x}_{1 T, \mathrm{~A}, 0}^{I}=a_{1} \cdot X^{I}+\frac{n a_{1}}{\pi} \omega_{0} t+\phi_{1 T, \mathrm{~A}, 0},
$$

where $\theta_{1}=-\frac{\pi}{2}$ and $X^{I}$ is an integer number along $x^{I}$-direction. $\phi_{1 T, \mathrm{~A}, 0}$ is a constant phase angle and $a_{1}$ is a fixed length that denotes the half pitch of the windings of $\mathrm{z}_{\mathrm{A}_{1}, 1}(\vec{x}, t)$. 
The level-1 $T$-type zero-lattice between two entangled vortex-membranes $\mathrm{B}_{1}$ and $\mathrm{B}_{2}$ for B-knot-crystal is obtained by level-1 T-type projection of B-knot-crystal,

$$
\hat{P}_{\theta_{1}}\left[z_{\mathrm{B}_{1}, 1}(\vec{x}, t)\right]=\hat{P}_{\theta}\left[z_{\mathrm{B}_{2}, 1}(\vec{x}, t)\right],
$$

i.e., $\xi_{\mathrm{B}_{1}, 1}(\vec{x}, t)=\xi_{\mathrm{B}_{2}, 1}(\vec{x}, t)$. The solution of the zero-lattice is obtained as

$$
\bar{x}_{1 T, \mathrm{~B}, 0}^{I}=a_{1} \cdot X^{I}+\frac{n a_{1}}{\pi} \omega_{0} t+\phi_{1 T, \mathrm{~B}, 0},
$$

where $\theta_{1}=-\frac{\pi}{2}$ and $X^{I}$ is an integer number along $x^{I}$-direction. $\phi_{1 T, \mathrm{~B}, 0}$ is a constant phase angle and $a_{1}$ is a fixed length that denotes the half pitch of the windings of $\mathrm{z}_{\mathrm{B}_{1}, 1}(\vec{x}, t)$.

In particular, there exist two intrinsic relationships between the number of $T$ type zeroes and level-2 $W$-type zeroes. One is twist-writhe locking relation for A-knot-crystal

$$
\zeta_{\left(\mathrm{A}_{1}, \mathrm{~A}_{2}\right), 1 \mathrm{D}}^{I}=W_{\left(\mathrm{A}_{1}, \mathrm{~A}_{2}\right), 1 \mathrm{D}}^{I}+T_{\left(\mathrm{A}_{1}, \mathrm{~A}_{2}\right), 1 \mathrm{D}}^{I} \equiv \text { const. }, \quad(I=x, y, z),
$$

where $\zeta_{\left(\mathrm{A}_{1}, \mathrm{~A}_{2}\right), 1 \mathrm{D}}^{I}, W_{\left(\mathrm{A}_{1}, \mathrm{~A}_{2}\right), 1 \mathrm{D}}^{I}$ and $T_{\left(\mathrm{A}_{1}, \mathrm{~A}_{2}\right), 1 \mathrm{D}}^{I}$ are linking number, writhe number, and twist number between vortex-membranes $A_{1}$ and $A_{2}$, respectively. Here, the number of level-1 $T$-type zeroes is equal to the twist number $T_{\left(\mathrm{A}_{1}, \mathrm{~A}_{2}\right), 1 \mathrm{D}}^{I}$ and the number of level-2 $W$-type zeroes is equal to be the writhe number $W_{\left(\mathrm{A}_{1}, \mathrm{~A}_{2}\right), 1 \mathrm{D}}^{I}$, respectively. The other is twist-writhe locking relation for B-knot-crystal

$$
\zeta_{\left(\mathrm{B}_{1}, \mathrm{~B}_{2}\right), 1 \mathrm{D}}^{I}=W_{\left(\mathrm{B}_{1}, \mathrm{~B}_{2}\right), 1 \mathrm{D}}^{I}+T_{\left(\mathrm{B}_{1}, \mathrm{~B}_{2}\right), 1 \mathrm{D}}^{I} \equiv \text { const. }, \quad(I=x, y, z),
$$

where $\zeta_{\left(\mathrm{B}_{1}, \mathrm{~B}_{2}\right), 1 \mathrm{D}}^{I}, W_{\left(\mathrm{B}_{1}, \mathrm{~B}_{2}\right), 1 \mathrm{D}}^{I}$ and $T_{\left(\mathrm{B}_{1}, \mathrm{~B}_{2}\right), 1 \mathrm{D}}^{I}$ are linking number, writhe number and twist number between vortex-membranes $\mathrm{B}_{1}$ and $\mathrm{B}_{2}$, respectively. Here, the number of level-1 $T$-type zeroes is equal to the twist number $T_{\left(\mathrm{B}_{1}, \mathrm{~B}_{2}\right), 1 \mathrm{D}}^{I}$ and the number of level-2 $W$-type zeroes is equal to be the writhe number $W_{\left(\mathrm{B}_{1}, \mathrm{~B}_{2}\right), 1 \mathrm{D}}^{I}$, respectively.

\subsubsection{3-level composite knot-crystal with $(\mathcal{N}=4, \mathcal{M}=3)$}

To generate a 3 -level composite knot-crystal with $(\mathcal{N}=4, \mathcal{M}=3)$, we wind a 2-level double-helix knot-crystal with $(\mathcal{N}=4, \mathcal{M}=3)$ along different spatial directions. As a result, there exists an additional $W$-type zero-lattice by $W$-type projection on the center membrane of A-knot-crystal and B-knot-crystal - the level-3 $W$-type zero-lattice. We use $\mathrm{z}_{3}(\vec{x}, t)$ to denote the center membranes of A-knot-crystal and B-knot-crystal.

So, the level-3 $W$-type zero-lattice of the 3-level composite knot-crystal with $(\mathcal{N}=4, \mathcal{M}=3)$ is obtained by level-3 $W$-type projection of center membranes of $\mathrm{z}_{3}(\vec{x}, t)$, i.e.,

$$
\hat{P}_{\theta_{3}}\left[\mathrm{z}_{3}(\vec{x}, t)\right]=0,
$$

that is $\xi_{3}(\vec{x}, t)=0$. The solution of zero-lattice is given by

$$
\bar{x}_{3 W, 0}^{I}(t)=a_{3} \cdot X^{I}-\frac{a_{3}}{\pi} \omega_{0} t+\phi_{3 W, 0},
$$


where $X^{I}$ is an integer along $x^{I}$-direction and $\theta_{3}=-\frac{\pi}{2} \cdot \phi_{3 W, 0}$ is a constant phase angle and $a_{3}$ is a fixed length that denotes the half pitch of the windings of $\mathrm{z}_{3}(\vec{x}, t)$.

Owing to the existence of an additional level-3 $W$-type zero-lattice, there exists an additional intrinsic relationship between the number of level-2 $T$-type zeroes and level-3 $W$-type zeroes,

$$
\zeta_{(\mathrm{A}, \mathrm{B}), 1 \mathrm{D}}^{I}=W_{(\mathrm{A}, \mathrm{B}), 1 \mathrm{D}}^{I}+T_{(\mathrm{A}, \mathrm{B}), 1 \mathrm{D}}^{I} \equiv \text { const. }, \quad(I=x, y, z)
$$

where $\zeta_{(\mathrm{A}, \mathrm{B}), 1 \mathrm{D}}^{I}, W_{(\mathrm{A}, \mathrm{B}), 1 \mathrm{D}}^{I}$ and $T_{(\mathrm{A}, \mathrm{B}), 1 \mathrm{D}}^{I}$ are linking number, writhe number and twist number, respectively. Here, the number of level-2 $T$-type zeroes is equal to the twist number $T_{(\mathrm{A}, \mathrm{B}), 1 \mathrm{D}}^{I}$ and the number of level-3 $W$-type zeroes is equal to be the writhe number $W_{(\mathrm{A}, \mathrm{B}), 1 \mathrm{D}}^{I}$, respectively. Because the sum of the number of level-2 $T$-type zeroes and level-3 $W$-type zeroes is invariant, when one winds two entangled vortex-membrane [a 2-level double-helix knot-crystal with $(\mathcal{N}=4, \mathcal{M}=3)$ ] into a 3-level winding knot-crystal with $(\mathcal{N}=4, \mathcal{M}=3)$, some level-2 $T$-type of zeroes are replaced by level-3 $W$-type zeroes. This is the substitution effect of level-2 T-type of zeroes by level-3 $W$-type zeroes.

\section{Emergent Quantum Field Theory for 1-Level Knot-Crystal with $(\mathcal{N}=1, \mathcal{M}=1)$}

From the above discussion, the winding number density becomes a physical quantity to characterize local windings of a helical vortex-membrane [1-level knot-crystal with $(\mathcal{N}=1, \mathcal{M}=1)]$ and the element of the helical vortex-membrane is knot with half winding number that corresponds to a crossing of a helical vortex-membrane and a straight line in its center. We then discuss the local fluctuations of a perturbative helical vortex-membrane with an extra knot and develop a theory to characterize its dynamics.

\subsection{Knot}

A knot of 1-level knot-crystal with $(\mathcal{N}=1, \mathcal{M}=1)$ is an anti-phase-changing along an arbitrary direction $\vec{e}$. As a result, each knot corresponds to a zero.

A $W$-type knot with a $W$-type zero is a half winding of 1-level knot-crystal with $(\mathcal{N}=1, \mathcal{M}=1)$. Figure 9 (a) is an example of a $W$-type knot. We then introduce the operation for the $1 \mathrm{D} \bar{W}$-type knot

$$
\hat{U}(\phi(x))=r_{0} \exp [i \phi(x) \cdot \hat{K}]
$$

on a constant complex field $z_{0}=0$ (we use [0] to denote the flat vortex-line) to generate a single $W$-type knot, i.e.,

$$
\hat{U}(\phi(x)) \cdot[0]=\mathrm{z}(x)=r_{0} e^{i \phi(x)} .
$$


Here, $\hat{K}=-i \frac{d}{d \phi}$ is knot number operator and

$$
\phi(x)=\left\{\begin{array}{ll}
\phi_{0} \mp \frac{\pi}{2}, & x \in\left(-\infty, x_{0}\right], \\
\phi_{0} \mp \frac{\pi}{2} \pm k_{0}\left(x-x_{0}\right), & x \in\left(x_{0}, x_{0}+a\right], \\
\phi_{0} \pm \frac{\pi}{2}, & x \in\left(x_{0}+a, \infty\right),
\end{array}\right\}
$$

where $+\left( \pm k_{0}\left(x-x_{0}\right)\right)$ denotes a clockwise winding and - denotes a counterclockwise winding.

The knot number for 1D $W$-type knot can be obtained by the following equation:

$$
\langle\hat{K}\rangle=\frac{1}{\pi r_{0}^{2}} \int \mathrm{z}^{*}(\phi(x)) \cdot \hat{K} \cdot \mathrm{z}(\phi(x)) d \phi,
$$

where $\mathrm{z}^{*}(x)$ is a complex conjugation of $\mathrm{z}(x)$. In physics, $\langle\hat{K}\rangle$ measures the total phase-changing for a knot with half winding number that can be regarded as an anti-phase domain wall along the given direction in a $1 \mathrm{D}$ complex field $\mathrm{z}(x)$, i.e., $\langle\hat{K}\rangle=1$. The knot density (the density of crossings) and the density of winding numbers are defined by $\rho_{\text {knot }}^{I}=\left\langle\frac{\hat{K}}{\Delta x}\right\rangle$ and $\rho_{\text {wind }}=2 \rho_{\text {knot }}=\left\langle\frac{2 \hat{K}}{\Delta x}\right\rangle$, respectively.

We call the extended object unified $W$-type knot. The definition can be generalized to $d$-dimensional $W$-type knot. The knot number for $d$-dimensional $W$-type knot along $x^{I}$-direction can be obtained by the following equation:

$$
\left\langle\hat{K}^{I}\right\rangle=\frac{1}{\pi r_{0}^{2}} \int \mathrm{z}^{*}\left(\phi\left(x^{I}\right)\right) \cdot \hat{K} \cdot \mathrm{z}\left(\phi\left(x^{I}\right)\right) d \phi^{I} .
$$

In physics, $\left\langle\hat{K}^{I}\right\rangle$ measures the total phase-changing for a knot with half winding number that can be regarded as an anti-phase domain wall along the given direction in a $1 \mathrm{D}$ complex field $\mathrm{z}\left(x^{I}\right)$, i.e., $\left\langle\hat{K}^{I}\right\rangle=1$. The knot density (the density of crossings) and the density of winding numbers are defined by $\rho_{\mathrm{knot}}^{I}=\left\langle\frac{\hat{K}^{I}}{\Delta x^{I}}\right\rangle$ and $\rho_{\text {wind }}^{I}=2 \rho_{\text {knot }}^{I}=\left\langle\frac{2 \hat{K}^{I}}{\Delta x^{I}}\right\rangle$, respectively. The total density of a knot is defined by

$$
\rho_{\mathrm{knot}}(\vec{x}, t)=\prod_{I} \rho_{\mathrm{knot}}^{I}\left(x^{I}, t\right)
$$

(a) Fragmentized $\boldsymbol{W}$-type knot: However, because $W$-type knot comes from the winding of a helical vortex-membrane, it is not a rigid object. Instead, it can split and be fragmentized. We then introduce the concept of "fragmentized $W$-type knot" by breaking a $W$-type knot into $N$ pieces $(N \rightarrow \infty)$, each of which is an identical $\frac{1}{N}$-knot with $\frac{\pi}{N}$ phase-changing. The function of Kelvin wave with a fragmentized $W$-type knot (a composite object of $N$ identical $\frac{1}{N}$-knot) is

$$
[z(\phi)]_{\text {fragment }, N}=\prod_{i=1}^{N} \hat{U}\left(\Delta \phi=\frac{\pi}{N},\left(x_{0}\right)_{i}\right) z_{0},
$$


where $N$ identical $\frac{1}{N}$-knots are at $\left(x_{0}\right)_{1},\left(x_{0}\right)_{2}, \ldots,\left(x_{0}\right)_{N}$. For each $\frac{1}{N}$-knot, the knot number $\langle\hat{K}\rangle$ is $\frac{1}{N}$ and the corresponding phase-changing is $\Delta \phi=\frac{\pi}{N}$. Thus, for a fragmentized knot, there also exists only a single knot solution and the knot number is conserved.

\subsection{Emergent quantum mechanics}

Knots can be regarded as quantum particles that obey emergent quantum mechanics and that the distribution of fragmentized knots is determined by Schrödinger equation.

The function of the Kelvin wave with a fragmentized knot describes the distribution of the $N$ identical $\frac{1}{N}$-knots and plays the role of the wavefunction in emergent quantum mechanics as

$$
\frac{[z(\phi)]_{\text {fragment }}}{z_{0}}=\sqrt{\Omega(\vec{x}, t)} e^{i \Delta \phi(\vec{x}, t)} \Longleftrightarrow \psi(\vec{x}, t)
$$

and $\Omega(\vec{x}, t)=\rho_{\text {knot }}(\vec{x}, t) \Longleftrightarrow n_{\text {knot }}(\vec{x}, t)$, where the function of the Kelvin wave with a fragmentized knot $\frac{[z(\phi)]_{\text {fragment }}}{z_{0}}$ becomes the wavefunction $\psi(\vec{x}, t)$ in emergent quantum mechanics; the angle $\Delta \phi(\vec{x}, t)$ becomes the quantum phase angle of the wavefunction; $\Omega(\vec{x}, t)$ is equal to the knot density $\rho_{\text {knot }}$ and thus becomes the probability density for finding a $\operatorname{knot} n_{\text {knot }}(\vec{x})$.

In emergent quantum mechanics, the projected energy and the projected momentum become operators for a fragmentized knot.

The projected momentum of a fragmentized knot on helical vortex-membrane with an excited Kelvin wave $\psi(\vec{x}, t)=\frac{1}{\sqrt{V}} e^{-i \Delta \omega t+i \Delta \vec{k} \cdot \vec{x}}$ is defined to be

$$
p_{\text {knot }}=\hbar_{\text {eff }} \Delta k
$$

where the effective Planck constant $\hbar_{\text {eff }}$ is obtained as the projected angular momentum of a knot

$$
\hbar_{\mathrm{eff}}=J_{\mathrm{knot}}=\frac{1}{2} \rho_{0} \kappa V_{P} r_{0}^{2} .
$$

Given the superposition principle of Kelvin waves, a generalized wavefunction is $\psi(\vec{x}, t)=\sum_{p} c_{p} \exp \left(\frac{-i E_{\mathrm{knot}} t+i \vec{p}_{\mathrm{knot}} \cdot \vec{x}}{\hbar_{\text {eff }}}\right)$. For an arbitrary wavefunction $\psi(\vec{x}, t)$, we have $\left\langle\vec{p}_{\text {knot }}\right\rangle=\int \vec{p}_{\text {knot }} \Omega(\vec{x}) d V=\int \psi^{*}(\vec{x}, t)\left(-i \hbar_{\text {eff }} \frac{d}{d \vec{x}}\right) \psi(\vec{x}, t) d V$. This result indicates that the projected momentum for a fragmentized knot becomes operator $\vec{p}_{\text {knot }} \rightarrow \hat{p}_{\text {knot }}=-i \hbar_{\text {eff }} \frac{d}{d \vec{x}}$. For a plane Kelvin wave $\psi(\vec{x}, t)=\frac{1}{\sqrt{V}} e^{-i \Delta \omega t+i \Delta \vec{k} \cdot \vec{x}}$, the projected energy of a fragmentized knot is

$$
E_{\mathrm{knot}}=J_{\mathrm{knot}} \Delta \omega=\hbar_{\mathrm{eff}} \Delta \omega
$$

Using a similar approach, one can see that the projected energy for a fragmentized knot becomes operator $E_{\mathrm{knot}} \rightarrow \hat{H}_{\mathrm{knot}}=i \hbar_{\mathrm{eff}} \frac{d}{d t}$. 


\subsection{Quantum Fermionic lattice model for knots}

Because the knots on 1-level knot-crystal with $(\mathcal{N}=1, \mathcal{M}=1)$ has only one chirality (we assume left-hand chirality), the effective quantum model is that of Weyl fermions. ${ }^{18}$

First, we discuss the statistics of a knot. In quantum mechanics, particles with wavefunction's antisymmetry under exchanging are called Fermions. To illustrate the Fermi statistics of knots, we define the Fermionic operator for knots with righthand chirality as $c^{\dagger}(\vec{x})=\hat{U}(\phi(\vec{x}))$. It is obvious that the wavefunction's antisymmetry by exchanging two knots is a result of the $\pi$-phase-changing nature of knots, i.e.,

$$
\hat{U}\left(\phi^{\prime}\left(\vec{x}^{\prime}\right)\right) \cdot \hat{U}(\phi(\vec{x}))=-\hat{U}(\phi(\vec{x})) \cdot \hat{U}\left(\phi^{\prime}\left(\vec{x}^{\prime}\right)\right) .
$$

As a result, knots obey Fermi statistics, $\left\{c^{\dagger}(\vec{x}), c^{\dagger}\left(\vec{x}^{\prime}\right)\right\}=0$.

Next, we derive the effective quantum field theory for Weyl fermions. A knot has two spin degrees of freedom $\uparrow$ or $\downarrow$ from the helicity degrees of freedom. The basis to define the microscopic structure of a knot is given by $|\uparrow\rangle,|\downarrow\rangle$. We define operator of knot states by the region of the phase angle of a knot: for the case of $\phi_{0} \bmod (2 \pi) \in(-\pi, 0]$, we have $c^{\dagger}|0\rangle$; for the case of $\phi_{0} \bmod (2 \pi) \in(0, \pi]$, we have $\left(c^{\dagger}|0\rangle\right)^{\dagger}$.

To characterize the energy cost from global winding, we use an effective Hamiltonian to describe the coupling between 2-knot states along $x^{I}$-direction on 3D SOC knot-crystal

$$
J c_{i}^{\dagger} T^{I} c_{i+e^{I}}
$$

with the annihilation operator of knots at the site $i, c_{i}=\left(\begin{array}{l}c_{\uparrow, i} \\ c_{\downarrow, i}\end{array}\right) . J$ is the coupling constant between two nearest-neighbor knots. According to the generalized translation symmetry, the transfer matrices $T^{I}$ along $x^{I}$-direction are defined by

$$
T^{I}=e^{i a\left(\hat{k}^{I} \cdot \sigma^{I}\right)} .
$$

Then, we get the total kinetic term as

$$
J \sum_{\langle i, j\rangle} c_{i}^{\dagger} T^{I} c_{i+e^{I}}+\text { h.c. }
$$

where $\langle i, j\rangle$ denotes the nearest-neighbor knots. Figure 10(a) is an illustration of entanglement pattern of a two-dimensional (2D) SOC knot-crystal with $(\mathcal{N}=1$, $\mathcal{M}=1$ ) and Fig. 10(d) is an illustration of a knot that changes entanglement along $x$ - and $y$-directions. In Fig. 10(a), each circle denotes a zero.

We then use path integral formulation to characterize the effective Hamiltonian for a knot-crystal as

$$
\int \mathcal{D} \psi^{\dagger}(t, \vec{x}) \mathcal{D} \psi(t) e^{i \mathcal{S} / \hbar}
$$




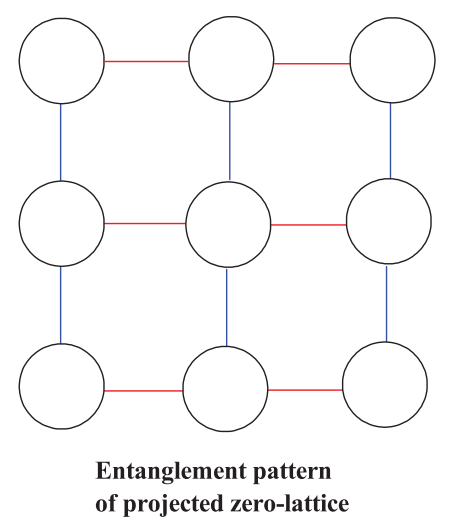

(a)

\section{Entanglement} matrix along x-direction: $\sigma_{\mathrm{x}}$ (b)

\section{Entanglement matrix along y-direction: $\sigma_{\mathrm{y}}$}

(c)

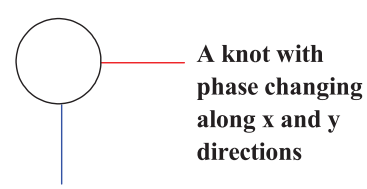

(d)

Fig. 10. (Color online) (a) An illustration of entanglement pattern of a 2D SOC knot-crystal with $(\mathcal{N}=1, \mathcal{M}=1)$. Each circle denotes a zero; (b) an illustration of entanglement matrix along $x$-direction; (c) an illustration of entanglement matrix along $y$-direction; (d) an illustration of knot that changes entanglement along $x$ - and $y$-directions.

where $\mathcal{S}=\int \mathcal{L} d t$ and $\mathcal{L}=i \sum_{i} \psi_{i}^{\dagger} \partial_{t} \psi_{i}-\mathcal{H}_{\text {coupling. }}$. To describe the knot states on $3 \mathrm{D}$ knot-crystal, we have introduced a two-component fermion field as $\psi(t, \vec{x})=$ $\left(\begin{array}{l}\psi_{\uparrow}(t, \vec{x}) \\ \psi_{\downarrow}(t, \vec{x})\end{array}\right)$, where $\uparrow, \downarrow$ label two spin degrees of freedom that denote the two possible winding directions along a given direction $\vec{e}$.

In continuum limit, we have

$$
\mathcal{H}_{\text {coupling }}=2 a J \sum_{k} \psi_{k}^{\dagger}\left[\sigma_{x} \cos k_{x}+\sigma_{y} \cos k_{y}+\sigma_{z} \cos k_{z}\right] \psi_{k}
$$

where the dispersion of knots is

$$
E_{\mathrm{A} / \mathrm{B}, k} \simeq c_{\mathrm{eff}}\left[\left(\vec{k}-\vec{k}_{0}\right) \cdot \boldsymbol{\sigma}\right],
$$

where $\vec{k}_{0}=\left(\frac{\pi}{2}, \frac{\pi}{2}, \frac{\pi}{2}\right)$ and $c_{\text {eff }}=2 a J$ is the velocity. In the following part, we ignore $\vec{k}_{0}$.

From the above equation, in the limit $|\vec{k}| \rightarrow 0$, we derive low-energy effective Hamiltonian as

$$
\begin{aligned}
\mathcal{H}_{3 \mathrm{D}} & \simeq 2 a J \sum_{k} \psi_{k}^{\dagger}(\boldsymbol{\sigma} \cdot \vec{k}) \psi_{k} \\
& =c_{\mathrm{eff}} \int \psi^{\dagger}(\boldsymbol{\sigma} \cdot \hat{k}) \psi d^{3} x .
\end{aligned}
$$

We then re-write the effective Hamiltonian to be

$$
\mathcal{H}_{3 \mathrm{D}}=\int\left(\psi^{\dagger} \hat{H}_{3 \mathrm{D}} \psi\right) d^{3} x
$$

and

$$
\hat{H}_{3 \mathrm{D}}=c_{\mathrm{eff}} \boldsymbol{\sigma} \cdot \vec{p}_{\mathrm{knot}},
$$


where $\vec{p}=\hbar_{\text {knot }} \vec{k}$ is the momentum operator. $c_{\text {eff }}$ plays the role of light speed where $a$ is a fixed length that denotes the half pitch of the windings on the knot-crystal.

The Schrödinger equation for knot becomes

$$
i \hbar_{\mathrm{eff}} \frac{d \psi(\vec{x}, t)}{d t}=\hat{H}_{3 \mathrm{D}} \psi(\vec{x}, t) .
$$

With the help of Schrödinger equation, we can predict the spacial distribution of fragmentized $\frac{1}{N}$-knots by varying the rotating velocity, $\omega_{0} \rightarrow \omega_{0}+\Delta \omega$. In the following parts, we set $\hbar_{\mathrm{knot}}=1$ and $c_{\mathrm{eff}}=1$.

\section{Emergent Quantum Field Theory for 1-Level Knot-Crystal with $(\mathcal{N}=2, \mathcal{M}=1)$}

The dynamic of $T$-type knot on a 1-level SOC knot-crystal with $(\mathcal{N}=2, \mathcal{M}=1)$ has been developed in Ref. 13. In Ref. 13, the low-energy effective Hamiltonian of knot has been obtained as

$$
\mathcal{H}_{3 \mathrm{D}}=\int\left(\Psi^{\dagger} \hat{H}_{3 \mathrm{D}} \Psi\right) d^{3} x
$$

and

$$
\hat{H}_{3 \mathrm{D}}=c_{\mathrm{eff}} \vec{\Gamma} \cdot \vec{p}_{\mathrm{knot}}+m_{\mathrm{knot}} c_{\mathrm{eff}}^{2} \Gamma^{5}
$$

where

$$
\begin{aligned}
& \Gamma^{5}=\tau^{x} \otimes \overrightarrow{1}, \quad \Gamma^{1}=\tau^{z} \otimes \sigma^{x}, \\
& \Gamma^{2}=\tau^{z} \otimes \sigma^{y}, \quad \Gamma^{3}=\tau^{z} \otimes \sigma^{z} .
\end{aligned}
$$

Here, $\vec{p}=\hbar_{\text {knot }} \vec{k}$ is the momentum operator. $\Psi=\left(\psi_{\mathrm{A}, \uparrow}^{*}, \psi_{\mathrm{B}, \uparrow}, \psi_{\mathrm{A}, \downarrow}^{*}, \psi_{\mathrm{B}, \downarrow}\right)$ is the generation operator of four-component fermions. $m_{\mathrm{knot}} c_{\mathrm{eff}}^{2}=2 \hbar_{\mathrm{knot}} \omega^{*}$ plays role of the mass of knots. In the following parts, we set $\hbar_{\text {knot }}=1$ and $c_{\text {eff }}=1$.

The low-energy effective Lagrangian of 3D SOC knot-crystal is

$$
\mathcal{L}_{3 \mathrm{D}}=i \Psi^{\dagger} \partial_{t} \Psi-\mathcal{H}_{3 \mathrm{D}}=\bar{\Psi}\left(i \gamma^{\mu} \hat{\partial}_{\mu}-m_{\mathrm{knot}}\right) \Psi,
$$

where $\bar{\Psi}=\Psi^{\dagger} \gamma^{0}, \gamma^{\mu}$ are the reduced Gamma matrices,

$$
\gamma^{1}=\gamma^{0} \Gamma^{1}, \quad \gamma^{2}=\gamma^{0} \Gamma^{2}, \quad \gamma^{3}=\gamma^{0} \Gamma^{3}
$$

and

$$
\gamma^{0}=\Gamma^{5}=\tau_{x} \otimes \overrightarrow{1}, \quad \gamma^{5}=i \gamma^{0} \gamma^{1} \gamma^{2} \gamma^{3}
$$

\section{Emergent Quantum Field Theory for 2-Level Knot-Crystal with $(\mathcal{N}=2, \mathcal{M}=2)$}

For 2-level winding knot-crystal with $(\mathcal{N}=2, \mathcal{M}=2)$, there are two types of zero-lattices: level-2 $W$-type zero-lattice and level-1 $T$-type zero-lattice. There are two types of zeroes or knots: level-2 $W$-type zero (knot) and level- $1 T$-type zero (knot). In principle,the level-2 $W$-type knot becomes Weyl fermion and the level-1 
T-type knot becomes Dirac fermion. In addition, we will show that the two types of knots are coupled by $\mathrm{SU}_{\text {weak }}(2)$ gauge field.

Due to the writhe-twist locking condition, there exists an intrinsic relationship between the number of level-1 $T$-type zeroes and that of level-2 $W$-type zeroes,

$$
\zeta_{(\mathrm{A}, \mathrm{B}), 1 \mathrm{D}}^{I}=W_{(\mathrm{A}, \mathrm{B}), 1 \mathrm{D}}^{I}+T_{(\mathrm{A}, \mathrm{B}), 1 \mathrm{D}}^{I} \equiv \text { const. }, \quad(I=x, y, z)
$$

where $\zeta_{(\mathrm{A}, \mathrm{B}), 1 \mathrm{D}}^{I}, W_{(\mathrm{A}, \mathrm{B}), 1 \mathrm{D}}^{I}$ and $T_{(\mathrm{A}, \mathrm{B}), 1 \mathrm{D}}^{I}$ are linking number, writhe number (the number of level-2 $W$-type zeroes) and twist number (the number of level-1 $T$-type zeroes), respectively. So, the topological number of level- $2 W$-type knot and that of level-1 $T$-type knot are all equal to the linking number between two vortexmembranes. According to the substitution effect of level-1 $T$-type zeroes by level-2 $W$-type zeroes, the properties of $W$-type knot and those of left-hand $T$-type knot are the same. This symmetry is characterized by $\mathrm{SU}_{\text {weak }}(2)$ gauge symmetry - an $\mathrm{SU}(2)$ gauge symmetry between Weyl fermions and Dirac fermions.

\subsection{Knots on 2-level knot-crystal with $(\mathcal{N}=2, \mathcal{M}=2)$}

In this paper, we only consider the knot dynamics in the limit of $\Delta_{\{1,2\}}=\frac{a_{2}}{a_{1}} \gg 1$. Now, owing to the existence of two types of zeroes (the level-2 $W$-type zeroes and the level-1 $T$-type zeroes), there exist two types of knots: the level-2 $W$-type knots and the level-1 T-type knots. So, a knot is defined by changing the half linking number, i.e.,

$$
\Delta \zeta_{(\mathrm{A}, \mathrm{B}), 1 \mathrm{D}}^{I}= \pm \frac{1}{2}
$$

For a level-2 $W$-type knot, the changing of half writhe number leads to the changing of half linking number, i.e.,

$$
\Delta \zeta_{(\mathrm{A}, \mathrm{B}), 1 \mathrm{D}}^{I}=\Delta W_{(\mathrm{A}, \mathrm{B}), 1 \mathrm{D}}^{I}= \pm \frac{1}{2}
$$

For a level-1 $T$-type knot, the changing of half twist number leads to the changing of half linking number, i.e.,

$$
\Delta \zeta_{(\mathrm{A}, \mathrm{B}), 1 \mathrm{D}}^{I}=T_{(\mathrm{A}, \mathrm{B}), 1 \mathrm{D}}^{I}= \pm \frac{1}{2}
$$

According to the substitution effect of level-1 $T$-type of zeroes by level-2 $W$-type zeroes, the property of the level- $2 W$-type knots is same as that of level- $1 T$-type knots.

\subsection{Low-energy effective model for two types of knots}

Firstly, we consider the low-energy effective model for level-1 $T$-type knots.

Figure 11(a) is an illustration of entanglement pattern of a 2D SOC knot-crystal with $(\mathcal{N}=2, \mathcal{M}=2)$. Each circle denotes a level-2 $W$-type zero. The number of dotted lines that connect the two circles is considered to be a very larger number 


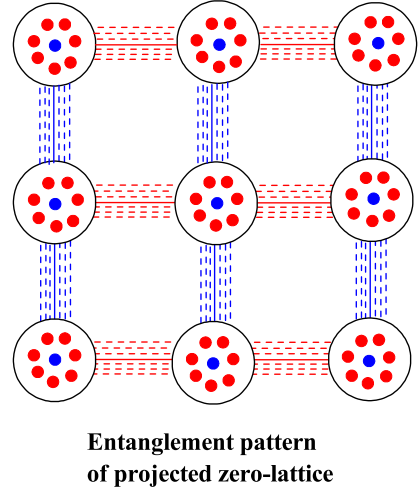

(a)
A $\mathrm{L}_{1}$ T knot with phase changing along $x$ and $y$ directions

(b)

$\mathbf{A ~ L}_{2} \mathbf{W}$ knot with
phase changing
along $x$ and $y$
directions

(c)

Fig. 11. (Color online) (a) An illustration of entanglement pattern of a 2D SOC knot-crystal with $(\mathcal{N}=2, \mathcal{M}=2)$. Each circle denotes a level-2 $W$-type zero. The number of dotted lines that connect the two circles is considered to be a very larger number (here the number is 6); (b) an illustration of level-1 T-type knot; (c) an illustration of level-2 $W$-type knot.

(here the number is 6). Figure 11(b) is an illustration of level-1 T-type knot and Fig. 11(c) is an illustration of level-2 $W$-type knot.

In the limit of $\Delta_{\{1,2\}}=\frac{a_{2}}{a_{1}} \gg 1$, the winding angles for $\mathrm{z}_{2}(\vec{x}, t)$ become dynamic coordinate. Along a given direction $\vec{e}$, the unit cell of the level-1 $T$-type knots turns into $a_{2}$. The position is determined by two kinds of values: $\vec{X}$ are numbers of zeroes for type-2 $W$-type zero-lattice, i.e.,

$$
\vec{X}=(X, Y, Z)=\frac{1}{\pi} \vec{\Phi}_{2}-\frac{1}{\pi} \vec{\Phi}_{2} \bmod \pi
$$

and $\varphi_{2}$ denote internal winding angles

$$
\varphi_{2}=\left(\varphi_{2, x}, \varphi_{2, y}, \varphi_{2, z}\right)=\vec{\Phi}_{2} \bmod \pi
$$

with $\varphi_{2, x}, \varphi_{2, y}, \varphi_{2, z} \in(0, \pi]$.

Therefore, on level-2 $W$-type zero-lattice, the effective Hamiltonian for level-1 $T$-type knots turns into

$$
\hat{H}_{3 \mathrm{D}}=\vec{\Gamma} \cdot \vec{p}_{\mathrm{knot}}+m_{\mathrm{knot}} \Gamma^{5}=\vec{\Gamma} \cdot \vec{p}_{X, \mathrm{knot}}+\vec{\Gamma} \cdot \vec{p}_{\varphi, \mathrm{knot}}+m_{\mathrm{knot}} \Gamma^{5},
$$

where $\vec{p}_{X}=\frac{1}{a_{2}} i \frac{d}{d \vec{X}}$ and $\vec{p}_{\varphi}=\frac{1}{a_{2}} i \frac{d}{d \vec{\varphi}}$. Because of $\varphi \in(0, \pi]$, quantum number of $\vec{p}_{\varphi}$ is angular momentum $\vec{L}_{\varphi}$ and the energy spectra are $\frac{1}{a_{2}}\left|\vec{L}_{\varphi}\right|$. If we focus on the low-energy physics $E \ll \frac{1}{a_{2}}$ (or $\vec{L}_{\varphi}=0$ ), we may get the low-energy effective Hamiltonian as

$$
\hat{H}_{3 \mathrm{D}} \simeq \vec{\Gamma} \cdot \vec{p}_{X, \mathrm{knot}}+m_{\mathrm{knot}} \Gamma^{5} .
$$

The low-energy effective Hamiltonian for level-1 $T$-type knots indicates that the knot-pieces of level-1 $T$-type knots have a uniform distribution inside the unit cell of level-2 $W$-type zero-lattice.

Next, we consider the low-energy effective model for level-2 $W$-type knots. 
The level-2 $W$-type knots change half linking number between two entangled vortex-membranes by winding globally. However, the level-2 $W$-type knots on a 2 level knot-crystal with $(\mathcal{N}=2, \mathcal{M}=2)$ are similar to the $W$-type knots on a 1-level knot-crystal with $(\mathcal{N}=1, \mathcal{M}=1)$ and have one chirality. We may set a $W$-type knot to be left-handed.

As a result, in the limit of $\Delta_{\{1,2\}}=\frac{a_{2}}{a_{1}} \gg 1$, every left-hand $W$-type knots can be regarded as a replacement of a left-hand $T$-type knot on a uniform knot-crystal. As a result, in long-wavelength limit, the left-hand $W$-type knot has same properties and we cannot distinguish a left-hand $W$-type knot and a left-hand $T$-type knot. If we focus on the low-energy physics $E \ll \frac{1}{a_{2}}$ (or $\vec{L}_{\varphi}=0$ ), we may get the low-energy effective Hamiltonian of left-hand $W$-type knot as

$$
\hat{H}_{3 \mathrm{D}} \simeq \vec{\sigma} \cdot \vec{p}_{X, \mathrm{knot}} .
$$

The low-energy effective Hamiltonian for level-2 $W$-type knots indicates that the knot-pieces of level-2 $W$-type knots also have a uniform distribution inside the unit cell of level-2 $W$-type zero-lattice.

Finally, the effective Lagrangian of the two types of knots $\psi_{L_{2} W}$ and $\psi_{L_{1} T}=$ $\left(\begin{array}{l}\psi_{L_{1} T, R} \\ \psi_{L_{1} T, L}\end{array}\right)$ becomes

$$
\begin{aligned}
\mathcal{L}_{\text {fermion }}(x)= & \bar{\psi}_{L_{2} W}(\vec{X}) i \gamma^{\mu} \partial_{\mu} \psi_{L_{2} W}(\vec{X})+\bar{\psi}_{L_{1} T}(\vec{X}) i \gamma^{\mu} \partial_{\mu} \psi_{L_{1} T}(\vec{X}) \\
& +m_{L_{1} T} \bar{\psi}_{L_{1} T}(\vec{X}) \psi_{L_{1} T}(\vec{X})
\end{aligned}
$$

There is no mass term for the left-hand level- $2 W$-type knots, i.e., $m_{L_{2} W}=0$.

\subsection{Emergent $\mathrm{SU}_{\text {weak }}(2)$ gauge symmetry}

Because in long-wavelength limit, the left-hand level-2 $W$-type knot and left-hand level-1 $T$-type knot have same properties, we cannot distinguish a left-hand $W$-type knot and a left-hand $T$-type knot. In addition, a left-hand $W$-type knot can change into a left-hand $T$-type knot and vice versa. So, $\mathrm{SU}_{\text {weak }}(2)$ gauge symmetry comes from the local symmetry of two types of knots in the unit cell of level- $2 W$-type zero-lattice.

For the knot-crystal, each left-hand knot (for example, a knot at $(\vec{x}, t)$ ) can be reorganized based on the basis $\left|\psi_{L_{2} W}\right\rangle,\left|\psi_{L_{1} T, L}\right\rangle$. Due to the symmetry of $\left|\psi_{L_{2} W}\right\rangle$, $\left|\psi_{L_{1} T, L}\right\rangle$, we can locally relabel the corresponding states of knots by $\left|\psi_{L_{2} W}^{\prime}\right\rangle$, $\left|\psi_{L_{1} T, L}^{\prime}\right\rangle$. The relationship between the two bases is

$$
\left(\begin{array}{c}
\left|\psi_{L_{2} W}^{\prime}\right\rangle \\
\left|\psi_{L_{1} T, L}^{\prime}\right\rangle
\end{array}\right)=U_{\mathrm{SU}_{\text {weak }}(2)}(\vec{x}, t)\left(\begin{array}{c}
\left|\psi_{L_{2} W}\right\rangle \\
\left|\psi_{L_{1} T, L}\right\rangle
\end{array}\right),
$$

where $U_{\mathrm{SU}_{\text {weak }}(2)}=e^{i \Theta(\vec{x}, t)}$ is the matrix of the representation of $\mathrm{SU}_{\text {weak }}(n)$ group. $\Theta(\vec{x}, t)=\sum_{a=1}^{3} \theta^{a}(\vec{x}, t) \tau^{a}$ and $\theta^{a}$ are a set of three constant parameters, and $\tau^{a}$ are three $2 \times 2$ matrices representing the three generators of the Lie algebra of $\mathrm{SU}_{\text {weak }}(2)$. This result leads to the concept of non-Abelian gauge symmetry. 
Thus, we have a local $\mathrm{SU}_{\text {weak }}(2)$ symmetry that denotes the two indistinguishable states of a knot with half linking number changing. According to the local $\mathrm{SU}_{\text {weak }}(2)$ gauge symmetry, the internal states of knots change as the following equation:

$$
\left(\begin{array}{c}
\left|\psi_{L_{2} W}\right\rangle \\
\left|\psi_{L_{1} T, L}\right\rangle
\end{array}\right) \rightarrow\left(\begin{array}{c}
\left|\psi_{L_{2} W}^{\prime}\right\rangle \\
\left|\psi_{L_{1} T, L}^{\prime}\right\rangle
\end{array}\right)=U_{\mathrm{SU}_{\text {weak }}(2)}(\vec{x}, t)\left(\begin{array}{c}
\left.\psi_{L_{2} W}\right\rangle \\
\left.\psi_{L_{1} T, L}\right\rangle
\end{array}\right)
$$

Due to the local $\mathrm{SU}_{\text {weak }}(2)$ gauge symmetry, the knot states $\left(\begin{array}{c}\left|\psi_{L_{2} W}\right\rangle \\ \left|\psi_{L_{1} T, L}\right\rangle\end{array}\right)$ and the knot states

$$
\left(\begin{array}{c}
\left|\psi_{L_{2} W}^{\prime}\right\rangle \\
\left|\psi_{L_{1} T, L}^{\prime}\right\rangle
\end{array}\right)=U_{\mathrm{SU}_{\text {weak }}(2)}(\vec{x}, t)\left(\begin{array}{c}
\left|\psi_{L_{2} W}\right\rangle \\
\left|\psi_{L_{1} T, L}\right\rangle
\end{array}\right)
$$

can be the same by changing the basis of states of knots.

As a result, to characterize the symmetry of the two types of knots, a left-hand level-2 $W$-type knot $\psi_{L_{2} W}$ and a left-hand level-2 $T$-type knot $\psi_{L_{1} T, L}$ make up a $\mathrm{SU}_{\text {weak }}(2) \operatorname{spinor}^{20}$

$$
\left(\begin{array}{c}
\psi_{L_{2} W} \\
\psi_{L_{1} T, L}
\end{array}\right)
$$

These considerations lead us to assign the left-handed components of the knots to doublets of $\mathrm{SU}_{\text {weak }}(2)$

$$
\psi_{L}=\frac{1}{2}\left(1+\gamma_{5}\right)\left(\begin{array}{c}
\psi_{L_{2} W} \\
\psi_{L_{1} T, L}
\end{array}\right) .
$$

In physics, there exists mixed knot state as $\alpha(\vec{x}, t) \psi_{L_{2} W}+\beta(\vec{x}, t) \psi_{L_{1} T, L}$ with $\alpha^{2}(\vec{x}, t)+\beta^{2}(\vec{x}, t)=1$. The right-handed components are assigned to singlets of $\mathrm{SU}_{\text {weak }}(2)$ that has level-1 $T$-type knot and we have

$$
\psi_{R}=\psi_{L_{1} T, R}=\frac{1}{2}\left(1-\gamma_{5}\right) \psi_{L_{1} T}
$$

As a result, we have

$$
\psi_{L} \rightarrow \psi_{L}^{\prime}=U_{\mathrm{SU}_{\text {weak }}(2)}(\vec{X}) \psi_{L}, \quad \psi_{R} \rightarrow \psi_{R},
$$

where $U_{\mathrm{SU}_{\text {weak }}(2)}(\vec{X})=e^{i \boldsymbol{\tau} \boldsymbol{\theta}(\vec{X})}$ and $\boldsymbol{\tau}$ are the three Pauli matrices.

\subsection{Emergent $\mathrm{SU}_{\text {weak }}(2)$ gauge theory}

The local symmetry of knot states leads to an $\mathrm{SU}_{\text {weak }}(2)$ gauge symmetry for composite knots (elementary fermions). The $\mathrm{SU}_{\text {weak }}(2)$ gauge symmetry for composite knots (elementary fermions) leads to emergent $\mathrm{SU}_{\text {weak }}(2)$ gauge theory for composite knot-crystal. To characterize the non-Abelian gauge symmetry, Yang and Mills in 1954 introduced "Yang-Mills field", $W_{\mu}(x)=\sum_{a=1}^{3} W_{\mu}^{a}(x) \tau^{a} \rightarrow W_{\mu}^{a} \tau^{a}$, $a=1,2,3$ that belong to the adjoint representation of $\mathrm{SU}_{\text {weak }}(2) . \frac{19}{} \mathrm{In}$ Ref. 19, neutrons and protons had been considered to be the doublets of $\mathrm{SU}_{\text {weak }}(2)$. However, 
in this paper, a left-hand level-2 $W$-type knot $\psi_{L_{2} W}$ and a left-hand level-1 T-type knot $\psi_{L_{1} T, L}$ are the doublets of $\mathrm{SU}_{\text {weak }}(2)$.

Due to the existence of the local $\mathrm{SU}_{\text {weak }}(2)$ gauge symmetry, there exists $\mathrm{SU}_{\text {weak }}(2)$ gauge field. Under changing knot states inside a composite knot, the knot state at site $\vec{j}$ changes as

$$
\psi_{L, \vec{j}} \rightarrow \psi_{L, \vec{j}}^{\prime}=\tilde{U}_{\vec{j}, \mathrm{SU}_{\text {weak }}(2)} \psi_{L, \vec{j}}
$$

Here $\tilde{U}_{\mathrm{SU}_{\text {weak }}(2)}$ is a changing of knot states based on certain basis $\left(\begin{array}{c}\psi_{L_{2} W} \\ \psi_{L_{1} T, L}\end{array}\right)$. After considering the local changing of basis induced by $U_{\vec{j}, \mathrm{SU}_{\text {weak }}(2)}$, the local coupling between two knot states changes, i.e.,

$$
J \psi_{L, \vec{j}}^{\dagger} T_{\vec{j}, \vec{j}^{\prime}} \psi_{L, \vec{j}^{\prime}} \rightarrow J\left(\psi_{L, \vec{j}} \tilde{U}_{\vec{j}, \mathrm{SU}_{\text {weak }}(2)}\right)^{\dagger} \cdot T_{\vec{j}, \vec{j}^{\prime}} \cdot\left(\tilde{U}_{\vec{j}^{\prime}, \mathrm{SU}_{\text {weak }}(2)} \psi_{L, \vec{j}^{\prime}}\right),
$$

where $T_{\vec{j}, \vec{j}^{\prime}}$ is translation operator from $\vec{j}$-site to $\vec{j}^{\prime}$-site. We define a vector field $W_{\vec{j}, \vec{j}^{\prime}}$ to characterize the local changing of basis

$$
e^{i g_{\text {weak }} W_{\vec{j}, \vec{j}^{\prime}}}=\left(\tilde{U}_{\vec{j}, \mathrm{SU}_{\text {weak }}(2)}\right)^{-1} \tilde{U}_{\vec{j}^{\prime}, \mathrm{SU}} \mathrm{weak}_{\text {weak }}(2),
$$

where $g_{\text {weak }}$ is coupling constant of $\mathrm{SU}_{\text {weak }}(2)$ non-Abelian gauge field. So, for perturbation case $W_{\vec{j}, \vec{j}^{\prime}} \sim 0$, we have

$$
i g_{\text {weak }} W_{\vec{j}, \vec{j}^{\prime}} \simeq\left(\delta \tilde{U}_{\vec{j}, \mathrm{SU}_{\text {weak }}(2)}^{-1}\right) \tilde{U}_{\vec{j}^{\prime}, \mathrm{SU}_{\text {weak }}(2)},
$$

where $\delta \tilde{U}_{\vec{j}, \mathrm{SU}_{\text {weak }}(2)}^{-1}=\tilde{U}_{\vec{j}, \mathrm{SU}_{\text {weak }}(2)}^{-1}-\tilde{U}_{\vec{j}^{\prime}, \mathrm{SU}_{\text {weak }}(2)}^{-1}$. The local coupling between two knot states becomes

$$
J \psi_{L, \vec{j}}^{\dagger} e^{i g_{\mathrm{weak}} W_{\vec{j}, \vec{j}^{\prime}}} T_{\vec{j}, \vec{j}^{\prime}} \psi_{L, \vec{j}^{\prime}}
$$

The total kinetic energy for knots becomes

$$
\hat{\mathcal{H}}_{\text {coupling }}=J \sum_{\left\langle\vec{j}, \vec{j}^{\prime}\right\rangle} J \psi_{L, \vec{j}}^{\dagger} e^{i g_{\text {weak }} W_{\vec{j}, \vec{j}^{\prime}}} T_{\vec{j}, \vec{j}^{\prime}} \psi_{L, \vec{j}^{\prime}}+\text { h.c. }
$$

It is obvious that the vector field $W_{\vec{j}, \vec{j}^{\prime}}$ that characterizes the local position perturbation of zero-lattice plays the role of $\mathrm{SU}_{\text {weak }}(2)$ gauge field. To illustrate the local $\mathrm{SU}_{\text {weak }}(2)$ gauge symmetry, we do a local $\mathrm{SU}_{\text {weak }}(2)$ gauge transformation $U_{\vec{j}, \mathrm{SU}_{\text {weak }}(2)}$ that is the transformation of basis of knot states, i.e.,

$$
\left(\begin{array}{c}
\left|\psi_{L_{2} W}\right\rangle \\
\left|\psi_{L_{1} T, L}\right\rangle
\end{array}\right) \rightarrow\left(\begin{array}{c}
\left|\psi_{L_{2} W}^{\prime}\right\rangle \\
\left|\psi_{L_{1} T, L}^{\prime}\right\rangle
\end{array}\right)=U_{\mathrm{SU}_{\text {weak }}(2)}(\vec{x}, t)\left(\begin{array}{c}
\left|\psi_{L_{2} W}\right\rangle \\
\left|\psi_{L_{1} T, L}\right\rangle
\end{array}\right) .
$$

Under a local $\mathrm{SU}_{\text {weak }}(2)$ gauge transformation, we have

$$
\psi_{L, \vec{j}} \rightarrow \psi_{L, \vec{j}}^{\prime}=U_{\vec{j}, \mathrm{SU}_{\text {weak }}(2)} \psi_{L, \vec{j}}
$$

and

$$
\begin{aligned}
g_{\text {weak }} W_{\vec{j}, \vec{j}^{\prime}} \rightarrow g_{\text {weak }} W_{\vec{j}, \vec{j}^{\prime}}^{\prime}= & g_{\text {weak }} U_{\vec{j}, \mathrm{SU}_{\text {weak }}(2)} W_{\vec{j}, \vec{j}^{\prime}}\left(U_{\vec{j}^{\prime}, \mathrm{SU}_{\text {weak }}(2)}\right)^{-1} \\
& +i\left(\delta U_{\vec{j}, \mathrm{SU}_{\text {weak }}(2)}^{-1}\right)\left(U_{\vec{j}, \mathrm{SU}_{\text {weak }}(2)}\right)^{-1}
\end{aligned}
$$


where $\delta U_{\vec{j}, \mathrm{SU}_{\text {weak }}(2)}^{-1}=\left(U_{\vec{j}, \mathrm{SU}_{\text {weak }}(2)}^{-1}-U_{\vec{j}^{\prime}, \mathrm{SU}_{\text {weak }}(2)}^{-1}\right)$. Here, we have used the following result:

$$
\begin{aligned}
e^{i g_{\text {weak }} W_{\vec{j}, \vec{j}^{\prime}}} & =\left(\tilde{U}_{\vec{j}, \mathrm{SU}_{\text {weak }}(2)}\right)^{-1} \tilde{U}_{\vec{j}^{\prime}, \mathrm{SU}_{\text {weak }}(2)} \rightarrow e^{i g_{\text {weak }} W_{\vec{j}, \vec{j}^{\prime}}^{\prime}} \\
& =U_{\vec{j}, \mathrm{SU}_{\text {weak }}(2)} e^{i g_{\text {weak }} W_{\vec{j}, \vec{j}^{\prime}}\left(U_{\vec{j}^{\prime}, \mathrm{SU}_{\text {weak }}(2)}\right)^{-1}} \\
& =U_{\vec{j}, \mathrm{SU}_{\text {weak }}(2)}\left(1+i g_{\text {weak }} W_{\vec{j}, \vec{j}^{\prime}}\right)\left(U_{\vec{j}^{\prime}, \mathrm{SU}_{\text {weak }}(2)}\right)^{-1} \\
& =U_{\vec{j}, \mathrm{SU}_{\text {weak }}(2)}\left(U_{\vec{j}^{\prime}, \mathrm{SU}_{\text {weak }}(2)}\right)^{-1}+i g_{\text {weak }} U_{\vec{j}, \mathrm{SU}_{\text {weak }}(2)} W_{\vec{j}, \vec{j}^{\prime}}\left(U_{\vec{j}^{\prime}, \mathrm{SU}_{\text {weak }}(2)}\right)^{-1} \\
& =1+i g_{\text {weak }} W_{\vec{j}, \vec{j}^{\prime}}^{\prime} \simeq e^{i g_{\text {weak }} W_{\vec{j}, \vec{j}^{\prime}}^{\prime}} .
\end{aligned}
$$

The total kinetic energy for knots turns into

$$
\hat{\mathcal{H}}_{\text {coupling }} \rightarrow \hat{\mathcal{H}}_{\text {coupling }}^{\prime}=J \sum_{\left\langle\vec{j}, \vec{j}^{\prime}\right\rangle}\left(\psi_{L, \vec{j}}^{\prime}\right)^{\dagger} e^{i g_{\text {weak }} W_{\vec{j}, \vec{j}^{\prime}}^{\prime}} T_{\vec{j}, \vec{j}^{\prime}} \psi_{L, \vec{j}}^{\prime}+\text { h.c. }
$$

The Hamiltonian does not change,

$$
\hat{\mathcal{H}}_{\text {coupling }}=\hat{\mathcal{H}}_{\text {coupling }}^{\prime} \text {. }
$$

On the other hand, the situation for tempo phase-changing is similar to that for spatial phase-changing. To characterize the tempo twist-writhe locking condition, we introduce a Lagrangian variable $W_{0, \vec{j}}$ to path integral formulation as

$$
W_{0, \vec{j}} \psi_{L, \vec{j}}^{\dagger} \psi_{L, \vec{j}} \cdot
$$

In continuum limit, we have, $U_{\vec{j}, \mathrm{SU}_{\text {weak }}(2)}(t) \rightarrow U_{\mathrm{SU}_{\text {weak }}(2)}(\vec{x}, t), W_{\vec{j}, \vec{j}^{\prime}} \rightarrow \vec{W}(x)$ and $W_{0, \vec{j}} \rightarrow W_{0}(x)$. The non-Abelian gauge symmetry is represented by

$$
\psi_{L}^{\prime} \rightarrow U_{\mathrm{SU}_{\text {weak }}(2)}(\vec{x}, t) \psi_{L}
$$

and

$$
\begin{aligned}
W_{\mu}(\vec{x}, t) \rightarrow & U_{\mathrm{SU}_{\text {weak }}(2)}(\vec{x}, t) W_{\mu}(\vec{x}, t)\left(U_{\mathrm{SU}_{\text {weak }}(2)}(\vec{x}, t)\right)^{-1} \\
& +\frac{i}{g_{\text {weak }}}\left(\partial_{\mu} U_{\mathrm{SU}_{\text {weak }}(2)}(\vec{x}, t)\right)\left(U_{\mathrm{SU}_{\text {weak }}(2)}(\vec{x}, t)\right)^{-1} .
\end{aligned}
$$

The gauge strength is defined by $W_{\mu \nu}$ as

$$
W_{\mu \nu}=\partial_{\mu} W_{\nu}-\partial_{\nu} W_{\mu}-i g_{\text {weak }}\left[W_{\mu}, W_{\nu}\right]
$$

or

$$
W_{\mu \nu}=W_{\mu \nu}^{a} \tau^{a}, \quad W_{\mu \nu}^{a}=\partial_{\mu} W_{\nu}^{a}-\partial_{\nu} W_{\mu}^{a}+g_{\text {weak }} f^{a b c} W_{\mu}^{b} W_{\nu}^{c} .
$$

The Lagrangian of Yang-Mills field can only be written as

$$
\mathcal{L}_{\mathrm{YM}}\left(\mathrm{SU}_{\text {weak }}(2)\right)=-\frac{1}{2} \operatorname{Tr}\left(W_{\mu \nu} W^{\mu \nu}\right)+g_{\text {weak }} \operatorname{Tr}\left(W_{\mu} j_{w}^{\mu}\right),
$$

where the weak current is

$$
j_{w-}^{\mu}=i \bar{\psi}_{L_{1} T, L} \gamma_{\mu} \psi_{L_{2} W}, \quad j_{w+}^{\mu}=i \bar{\psi}_{L_{2} W} \gamma_{\mu} \psi_{L_{1} T, L}
$$


The Lagrangian density $\mathcal{L}_{\mathrm{YM}}\left(\mathrm{SU}_{\text {weak }}(2)\right)$ is invariant under the gauge transformations with an $x$-dependent $U_{\mathrm{SU}_{\text {weak }}(2)}(\vec{x}, t)$.

Finally, we write down the effective Lagrangian of $\mathrm{SU}_{\text {weak }}(2)$ gauge theory

$$
\mathcal{L}=\operatorname{Tr}\left(\bar{\psi}_{L} i \gamma^{\mu}\left(\partial_{\mu}-i g_{\text {weak }} W_{\mu}\right) \psi_{L}\right)+\bar{\psi}_{R} i \gamma^{\mu} \partial_{\mu} \psi_{R}-\operatorname{Tr}\left(\frac{1}{2} W_{\mu \nu} W^{\mu \nu}\right),
$$

where $W_{\mu}$ denotes the gauge fields associated to $\mathrm{SU}_{\text {weak }}(2)$, respectively, of which the corresponding field strengths are $W_{\mu \nu}$. Because linking number of composite knot-crystal can only be changed for left-hand knots via changing the writhe number, the charged $W$ 's couple only to the left-handed components of the two types of knots.

In physics, the $\mathrm{SU}_{\text {weak }}(2)$ gauge field that couples the level-2 $W$-type knots and the level-1 $T$-type knots characterizes the interaction by exchanging the fluctuations of writhe number density on level-2 $W$-type zero-lattice. The nondiagonal fluctuations of $\mathrm{SU}_{\text {weak }}(2)$ gauge theory come from the density fluctuations of writhe number and the diagonal fluctuations of $\mathrm{SU}_{\text {weak }}(2)$ gauge theory come from the phase fluctuations of the zeroes ( $T$-type or $W$-type) inside the unit cell of level-2 $W$-type zero-lattice. Figure 12 is an illustration of the theoretical structure of emergent $\mathrm{SU}_{\text {weak }}(2)$ non-Abelian gauge symmetry and non-Abelian gauge fields.

\subsection{Higgs mechanism and spontaneous symmetry breaking}

In this part, we focus on leapfrogging motion of the composite knot-crystal of which the wavefunctions of level-1 $T$-type knots become time-dependent with fixed angular

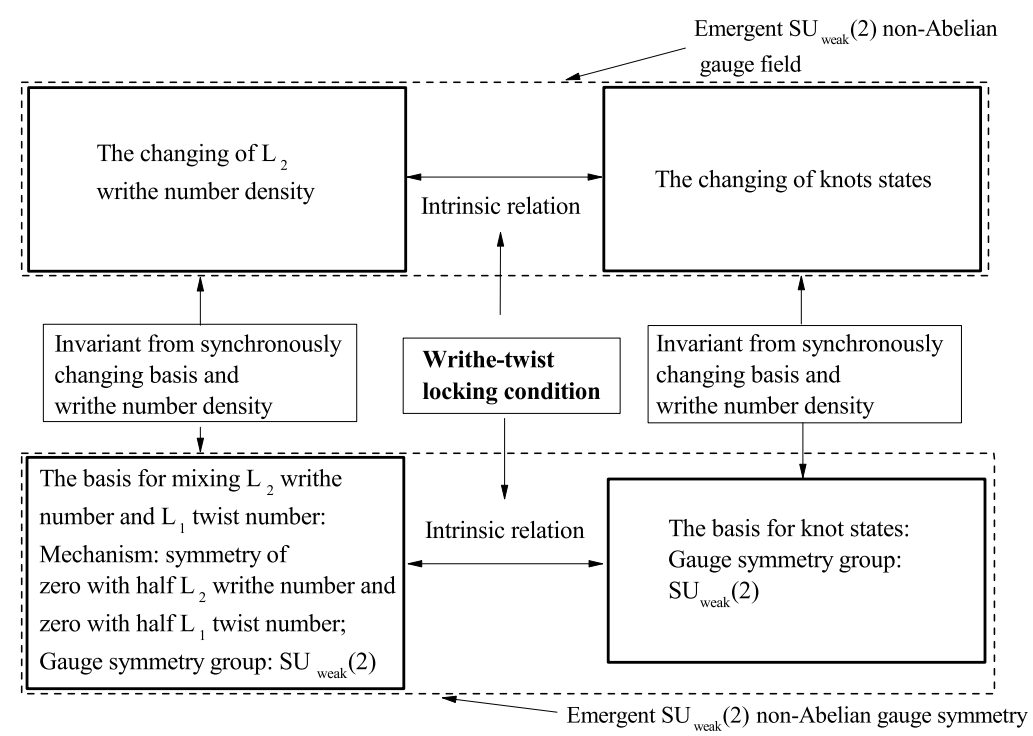

Fig. 12. An illustration of the theoretical structure of emergent $\mathrm{SU}_{\text {weak }}(2)$ non-Abelian gauge symmetry and non-Abelian gauge fields. 
velocity $\omega^{*}$ :

$$
\psi_{L_{1} T, A}(\vec{X}, t) \leftrightarrow \psi_{L_{1} T, B}^{*}(\vec{X}, t) .
$$

We then consider the fluctuating angular velocity of leapfrogging motion

$$
\omega^{*} \rightarrow \omega(\vec{X}, t),
$$

that plays the role of Higgs field $\Phi(\vec{X}, t), \underline{21}$ i.e.,

$$
\omega(\vec{X}, t) \leftrightarrow \Phi(\vec{X}, t) / 2
$$

And the condensation of Higgs field $\langle\Phi(\vec{X}, t)\rangle \neq 0$ corresponds to a finite leapfrogging angular velocity of the knot-crystal $\omega^{*} \neq 0$. It is the leapfrogging motion that gives masses to knots. Due to chirality, without leapfrogging motion left-hand level-2 $W$-type knot does not obtain mass.

We then study the properties of leapfrogging field $\omega(\vec{X}, t)$ (that is really the Higgs field $\Phi(\vec{X}, t) / 2)$. The effect of leapfrogging motion is to change $\psi_{L_{1} T, L}(\vec{X}, t)$ (that is $\left.\psi_{L_{1} T, A}\right)$ to $\psi_{L_{1} T, R}(\vec{X}, t)$ (that is $\left.\psi_{L_{1} T, B}^{*}\right)$ and there appears an extra term in Hamiltonian as

$$
\psi^{\dagger} \omega(\vec{X}, t) \tau_{x} \psi=\psi_{L_{1} T, R}^{\dagger} \omega(\vec{X}, t) \psi_{L_{1} T, L}
$$

On the other hand, due to

$$
\psi_{L}(\vec{X}) \rightarrow U_{\mathrm{SU}_{\text {weak }}(2)} \psi_{L}(\vec{X}), \quad \psi_{R}(\vec{X}) \rightarrow \psi_{R}(\vec{X}),
$$

$\omega(\vec{X}, t)$ must be an $\mathrm{SU}_{\text {weak }}(2)$ complex doublet as

$$
\omega(\vec{X}, t)=\left(\begin{array}{c}
\phi^{+} \\
\phi^{0}
\end{array}\right), \quad \omega(\vec{X}, t) \rightarrow U_{\mathrm{SU}_{\text {weak }}(2)} \omega(\vec{X}, t) .
$$

Next, we write down an effective Lagrangian of the leapfrogging field $\omega(\vec{X}, t)$. Because the leapfrogging field $\omega(\vec{X}, t)$ is an $\mathrm{SU}_{\text {weak }}(2)$ complex doublet, we get the kinetic term of leapfrogging field $\omega(\vec{X}, t)$, that is

$$
\left|\left(\partial_{\mu}-i g_{\text {weak }} \frac{\tau}{2} \cdot \vec{W}_{\mu}\right) \omega(\vec{X}, t)\right|^{2}
$$

To obtain the finite leapfrogging angular velocity, we add a phenomenological potential term $V(\omega(\vec{X}, t))$. Finally, by adding Yukawa coupling between the leapfrogging field and fermions, the full Lagrangian of leapfrogging field $\omega(\vec{X}, t)$ is given by

$$
\mathcal{L}_{\text {Higgs }}=\left|\left(\partial_{\mu}-i g_{\text {weak }} \frac{\tau}{2} \cdot \vec{W}_{\mu}\right) \omega(\vec{X}, t)\right|^{2}-V(\omega(\vec{X}, t))+2 \bar{\psi} \omega(\vec{X}, t) \psi+\text { h.c. }
$$

A finite leapfrogging angular velocity is given by minimizing $\omega(\vec{X}, t)$ of which the expected value is $\omega^{*}$. Then, the weak gauge symmetry is spontaneously broken, we get a finite leapfrogging angular velocity:

$$
\langle\omega(\vec{X}, t)\rangle=\left(\begin{array}{c}
0 \\
\omega^{*}
\end{array}\right)+\delta \omega(\vec{X}, t)
$$

The finite leapfrogging angular velocity plays the role of Higgs condensation. Under Higgs condensation, there exists Higgs mechanism. The Higgs mechanism breaks the 
original gauge symmetry according to $\mathrm{SU}_{\text {weak }}(2) \rightarrow \mathrm{Z} 2$. As a result, the $\mathrm{SU}_{\text {weak }}(2)$ gauge fields obtain masses from the following terms ${ }^{22}$ :

$$
\frac{1}{2}\left(\omega^{*}\right)^{2}\left(g_{\text {weak }}\right)^{2}\left(W_{\mu} W^{\mu}\right) .
$$

The mass for the charged vector bosons $W_{\mu}$ is $m_{W}=\omega^{*} g_{\text {weak }}$.

Before considering Higgs condensation or $\omega^{*}=0$, the low-energy effective Lagrangian density is

$$
\begin{aligned}
\mathcal{L}_{\mathrm{SM}}= & \operatorname{Tr} \bar{\psi}_{L} i \gamma^{\mu}\left(\partial_{\mu}-i g_{\text {weak }} \frac{\boldsymbol{\tau}}{2} \cdot \vec{W}_{\mu}\right) \psi_{L}+\bar{\psi}_{R} i \gamma^{\mu} \partial_{\mu} \psi_{R}-\operatorname{Tr} \frac{1}{2} W_{\mu \nu} W^{\mu \nu} \\
& +\left|\left(\partial_{\mu}-i g_{\text {weak }} \frac{\boldsymbol{\tau}}{2} \cdot \vec{W}_{\mu}\right) \omega\right|^{2}-V(\omega)+2 \bar{\psi}_{L} \omega \psi_{R}+\text { h.c. }
\end{aligned}
$$

After considering the Higgs condensation $\omega^{*} \neq 0$, we have the low energy effective Lagrangian as

$$
\begin{aligned}
\mathcal{L}_{\mathrm{SM}}= & \operatorname{Tr} \bar{\psi}_{L} i \gamma^{\mu}\left(\partial_{\mu}-i g_{\text {weak }} \frac{\boldsymbol{\tau}}{2} \cdot \vec{W}_{\mu}\right) \psi_{L}+\bar{\psi}_{R} i \gamma^{\mu} \partial_{\mu} \psi_{R}+m_{T} \bar{\psi}_{T} \psi_{T} \\
& -\operatorname{Tr} \frac{1}{2} \operatorname{Tr} W_{\mu \nu} W^{\mu \nu}+\frac{1}{2}\left(\omega^{*}\right)^{2}\left(g_{\text {weak }}\right)^{2} \operatorname{Tr} W_{\mu} W^{\mu} \\
& +\left|\partial_{\mu} \omega\right|^{2}+m_{\text {Higgs }}|\omega|^{2}+\cdots
\end{aligned}
$$

A finite leapfrogging angular velocity creates a mass term for the level-1 $T$-type knots, leaving the $W$-type massless,

$$
m_{T}=2 \omega^{*}, \quad m_{W}=\omega^{*} g_{\text {weak }} .
$$

In addition, the leapfrogging field also has mass $m_{\text {Higgs }}$. This is an $\mathrm{SU}_{\text {weak }}(2)$ gauge theory with Higgs mechanism due to spontaneous symmetry breaking.

\section{Emergent Quantum Field Theory for 2-Level Composite Knot-Crystal with $(\mathcal{N}=4, \mathcal{M}=2)$}

In this part, we will derive the low-energy effective model for knots on the composite knot-crystal with $(\mathcal{N}=4, \mathcal{M}=2)$. The composite knots correspond to elementary particles in particle physics, including electron and quarks.

\subsection{Composite knots — definition and classification}

For 2-level composite knot-crystal with $(\mathcal{N}=4, \mathcal{M}=2)$, there are five zerolattices: level-2 $W$-type zero-lattice for A-knot-crystal, level-2 $W$-type zero-lattice for B-knot-crystal, level-2 T-type zero-lattice between A-knot-crystal and B-knotcrystal, level-1 $T$-type zero-lattice between two entangled vortex-membranes $\mathrm{A}_{1}$ and $\mathrm{A}_{2}$ for A-knot-crystal, level-1 $T$-type zero-lattice between two entangled vortexmembranes $\mathrm{B}_{1}$ and $\mathrm{B}_{2}$ for B-knot-crystal.

The knots for the composite knot-crystal are defined to correspond to the level-2 $T$-type zeroes by level-2 $T$-type projection between A-knot-crystal and 
B-knot-crystal. The level-2 T-type knots have four degrees of freedom, two spin degrees of freedom and two vortex degrees of freedom. For knot on a composite knot-crystal, the linking number $\zeta_{1 \mathrm{D}}^{I}$ along given direction is changed by $\pm \frac{1}{2}$, i.e., $\delta \zeta_{1 \mathrm{D}}^{I}= \pm \frac{1}{2}$. A knot (an anti-knot) removes (or adds) a projected zero of level-2 $T$-type zero-lattice that corresponds to remove (or adds) half of the "lattice unit" on the level-2 $T$-type zero-lattice according to $\Delta x_{i}= \pm a_{2}$.

However, by trapping different internal zeroes, there exist different types of level-2 $T$-type knots. We use the following number series to label different types of knots:

$$
\left[n_{L_{2}}, n_{L_{1}}\right]
$$

where $n_{L_{2}}$ is the number changing of half linking of level-2 entangled knot-crystals that is equal to the number of level-2 $T$-type zeroes $n_{L_{2} T}$ as

$$
n_{L_{2}}=n_{L_{2} T}
$$

$n_{L_{1}}$ is the number changing of half linking of level-1 entangled vortex-membranes that is equal to the sum of the number of level-1 $T$-type zeroes $n_{2 T}$ and the number of level-2 $W$-type zeroes $n_{L_{2} W}$,

$$
n_{L_{1}}=n_{L_{1} T}+n_{L_{2} W} .
$$

For different types of knots, due to trapping half linking number of two entangled knot-crystals, we must have

$$
n_{L_{2} T} \equiv 1
$$

So, the classification of the knot type is based on the internal half linking number $n_{L_{1}}$ (the linking number of level-1 entangled vortex-membranes). For a 2-level composite knot-crystal with $(\mathcal{N}=4, \mathcal{M}=2)$ with an integer hierarchy number $\Delta_{\{1,2\}}=n$, there are $n$ different types of knots with $[1,0],[1,1], \ldots[1, n-1]$. The composite knots with $[1, n]$ correspond to electrons in particle physics and the composite knots with $\left[1, n_{k}\right]\left(n_{k}\right.$ is an integer number, $\left.0<n_{k}<n\right)$ correspond to quarks.

An object with level-2 half linking number is a knot and an object with level1 half linking number is an internal-knot. For a free level-2 composite knot with changing $\Delta n_{L_{2}}=1$, the effective Planck constant is

$$
\hbar_{L_{2} T}=\frac{1}{2} \rho_{0} \kappa V_{P} r_{2}^{2}
$$

For a free level-1 internal knot with changing $\Delta n_{L_{1}}=1$, the effective Planck constant is

$$
\hbar_{L_{1} T}=\frac{1}{2 n} \rho_{0} \kappa V_{P} r_{1}^{2}
$$

As a result, the effective Planck constant for different knots depends on the number of internal zeroes $n_{k}$,

$$
\hbar_{\left[1, n_{k}\right]}=\hbar_{L_{2} T}-n_{k} \hbar_{L_{1} T}=\frac{1}{2} \rho_{0} \kappa V_{P} r_{2}^{2}-\frac{n_{k}}{2 n} \rho_{0} \kappa V_{P} r_{1}^{2} .
$$


In this paper, we focus on the case of weak coupling limit $\left(r_{1} \ll r_{2}\right)$ and have

$$
\hbar_{\left[1, n_{k}\right]} \simeq \hbar_{L_{2} T}
$$

In general, the quantum state of a composite knot with $\left[1, n_{i}\right]$ is denoted by

$$
\psi_{L_{2} T, n_{i}, \tau, \sigma}^{\dagger}(\vec{x}, t)|\mathrm{vac}\rangle,
$$

where $\tau=\mathrm{A} / \mathrm{B}$ labels the vortex index, $\sigma=\uparrow / \downarrow$ labels spin degrees of freedom. A knot with $\left[1, n_{i}\right]$ is a composite object with a knot and $n_{i}$ internal knots. Because the knot with $[1, n]$ corresponds to electron, its quantum state is denoted by

$$
\psi_{L_{2} T, n, \tau, \sigma}^{\dagger}(\vec{x}, t)|\operatorname{vac}\rangle=e_{\tau, \sigma}^{\dagger}(\vec{x}, t)|\operatorname{vac}\rangle .
$$

There are $n$ internal knots inside the electron. Except for electrons, other types of knots correspond to quarks with $n_{i}$ internal knots of which quantum states are denoted by

$$
\psi_{L_{2} T, n_{i} \neq n, \tau, \sigma}^{\dagger}(\vec{x}, t)|\operatorname{vac}\rangle=q_{\tau, \sigma}^{\dagger}(\vec{x}, t)|\operatorname{vac}\rangle .
$$

We then show several 2-level composite knot-crystals with $(\mathcal{N}=4, \mathcal{M}=2)$.

For the case of $n=1$, there exists one type of knot without internal additional zero $\left(n_{k}=0\right)$ that is just electron.

For the case of $n=2$, except for electrons, there exists one type of quark with one internal additional zero (or an extra internal knot), $n_{k}=n_{\text {quark }}=1$. Due to the existence of two "sites" inside the knots, the internal additional zero has two degenerate internal states that are described by

$$
\psi_{L_{2} T, n_{i}=1, \tau, \sigma}^{\dagger}(\vec{x}, t)|\operatorname{vac}\rangle=q_{\tau, \sigma}^{\dagger}(\vec{x}, t)|\operatorname{vac}\rangle
$$

or

$$
\left(\begin{array}{l}
\psi_{L_{2} T, n_{i}=1,1, \tau, \sigma}^{\dagger}(\vec{x}, t)|\mathrm{vac}\rangle \\
\psi_{L_{2} T, n_{i}=1,2, \tau, \sigma}^{\dagger}(\vec{x}, t)|\mathrm{vac}\rangle
\end{array}\right)=\left(\begin{array}{c}
q_{1, \tau, \sigma}(\vec{x}, t)|\mathrm{vac}\rangle \\
q_{2, \tau, \sigma}(\vec{x}, t)|\mathrm{vac}\rangle
\end{array}\right) .
$$

For the case of $n=3$, except for electrons, there exists two types of quarks, $u$ quark and $d$-quark. The $d$-quarks are composite knots with one internal additional zero (or an extra internal knot), $n_{k}=n_{d \text {-quark }}=1$, of which there are three degenerate states described by

$$
\psi_{L_{2} T, n_{i}=1, \tau, \sigma}^{\dagger}(\vec{x}, t)|\mathrm{vac}\rangle=d_{\tau, \sigma}^{\dagger}(\vec{x}, t)|\mathrm{vac}\rangle
$$

or

$$
\left(\begin{array}{l}
\psi_{L_{2} T, n_{i}=1,1, \tau, \sigma}^{\dagger}(\vec{x}, t)|\mathrm{vac}\rangle \\
\psi_{L_{2} T, n_{i}=1,2, \tau, \sigma}^{\dagger}(\vec{x}, t)|\mathrm{vac}\rangle \\
\left.\psi_{L_{2} T, n_{i}=1,3, \tau, \sigma}^{\dagger}(\vec{x}, t) \mathrm{vac}\right\rangle
\end{array}\right)=\left(\begin{array}{l}
d_{1, \tau, \sigma}(\vec{x}, t)|\mathrm{vac}\rangle \\
d_{2, \tau, \sigma}(\vec{x}, t)|\mathrm{vac}\rangle \\
d_{3, \tau, \sigma}(\vec{x}, t)|\mathrm{vac}\rangle
\end{array}\right) .
$$

The three degenerate states of $d$-quarks are called red $d$-quark, blue $d$-quark and green $d$-quark, respectively. The $u$-quarks are composite knots with 2 internal zeroes (or two extra internal knots), $n_{k}=n_{u \text {-quark }}=2$,of which the three degenerate 
states are described by

$$
\psi_{L_{2} T, n_{i}=2, \tau, \sigma}^{\dagger}(\vec{x}, t)|\operatorname{vac}\rangle=u_{\tau, \sigma}^{\dagger}(\vec{x}, t)|\operatorname{vac}\rangle
$$

or

$$
\left(\begin{array}{l}
\psi_{L_{2} T, n_{i}=2,1, \tau, \sigma}^{\dagger}(\vec{x}, t)|\mathrm{vac}\rangle \\
\psi_{L_{2} T, n_{i}=2,2, \tau, \sigma}^{\dagger}(\vec{x}, t)|\operatorname{vac}\rangle \\
\psi_{L_{2} T, n_{i}=2,3, \tau, \sigma}^{\dagger}(\vec{x}, t)|\operatorname{vac}\rangle
\end{array}\right)=\left(\begin{array}{l}
u_{1, \tau, \sigma}(\vec{x}, t)|\operatorname{vac}\rangle \\
u_{2, \tau, \sigma}(\vec{x}, t)|\operatorname{vac}\rangle \\
u_{3, \tau, \sigma}(\vec{x}, t)|\operatorname{vac}\rangle
\end{array}\right) .
$$

The three degenerate states of $u$-quarks are called red $u$-quark, blue $u$-quark and green $u$-quark, respectively.

\subsection{Emergent Dirac model}

In this part, we discuss the emergent quantum field theory for composite knots without taking into account the dynamics of internal zeroes. For different types of knots on 2-level knot-crystal with $(\mathcal{N}=4, \mathcal{M}=2)$, there also exist two types of energy costs - the kinetic term and the mass term from leapfrogging motion. As a result, the low-energy effective model is similar to that of knot on 1-level knot-crystal with $(\mathcal{N}=2, \mathcal{M}=1)$.

For simplicity, we take the case of $n=3$ as an example. By introducing operator representation, we use the traditional effective Hamiltonian to describe the dynamics of composite knot-crystal as

$$
\hat{\mathcal{H}}_{\text {coupling }}=\frac{J}{2} \sum_{\langle i j\rangle} \psi_{i}^{\dagger} T_{i j} \psi_{j}+\text { h.c. }
$$

where $T_{i j}$ is translation operator from $i$-site to $j$-site,

$$
\psi_{i}=\left(\begin{array}{c}
e_{i} \\
u_{i} \\
d_{i}
\end{array}\right)
$$

and

$$
u_{i}=\left(\begin{array}{c}
u_{1, i} \\
u_{2, i} \\
u_{3, i}
\end{array}\right), \quad d_{i}^{\dagger}=\left(\begin{array}{c}
d_{1, i} \\
d_{2, i} \\
d_{3, i}
\end{array}\right)
$$

The seven types of knot operators denoted by $e_{i}, u_{1, i}, u_{2, i}, u_{3, i}, d_{1, i}, d_{2, i}, d_{3, i}$ have four degrees of freedom, two spin degrees of freedom and two vortex (or chiral) degrees of freedom.

After considering the leapfrogging motion, we write down the low-energy effective Lagrangian as

$$
\mathcal{L}=i \int \Psi^{\dagger} \partial_{t} \Psi d^{3} x-\hat{\mathcal{H}}_{3 \mathrm{D}}
$$


where

$$
\hat{\mathcal{H}}_{3 \mathrm{D}} \simeq \int \Psi^{\dagger}\left[\left(\tau_{z} \otimes \boldsymbol{\sigma} \otimes \mathbf{1}\right) \cdot \hat{k}\right] \Psi d^{3} x+2 \omega^{*} \int \Psi^{\dagger}\left[\tau_{x} \otimes \overrightarrow{1} \otimes \mathbf{1}\right] \Psi d^{3} x,
$$

where 1 is a $7 \times 7$ matrix and $\overrightarrow{1}$ is $2 \times 2$ unit matrix.

Finally, the Lagrangian of Dirac fermion of composite knot-crystal is derived by

$$
\mathcal{L}_{\text {fermion }}(x)=\bar{e} i \gamma^{\mu} \partial_{\mu} e+\bar{d} i \gamma^{\mu} \partial_{\mu} d+\bar{u} i \gamma^{\mu} \partial_{\mu} u+m_{e} \bar{e} e+m_{d} \bar{d} d+m_{u} \bar{u} u .
$$

Due to leapfrogging process, all fermionic elementary particles have finite mass,

$$
m_{e}=m_{d}=m_{u}=2 \omega^{*} .
$$

\subsection{Emergent $U(1)$ gauge field}

Gauge field is an important concept in physics. By understanding electromagnetism, Maxwell introduced the concept of the electromagnetic field and believed that the propagation of light required a medium for the waves, namely, the luminiferous aether. Quantum gauge field theory, particularly the QED is an extremely successful theory of electromagnetic interaction that agrees with experiments very well. The key point of QED is the existence of $\mathrm{U}(1)$ gauge symmetry.

For 2-level knot-crystal with $(\mathcal{N}=4, \mathcal{M}=2)$ and $n$ internal knots, there are $n$ types of composite knots that are topological excitations in a composite knotcrystal. Except for the topological excitations (the knots), there exist collective excitations of internal twistings - gauge fields. The phase fluctuations of internal zero-lattice are $\mathrm{U}(1)$ gauge field.

\subsubsection{Phase-changing from writhe-twist locking condition}

In this section, we study the knot dynamics and entanglement evolution of internal twisting after considering the twist-writhe locking condition.

There exist two intrinsic relationships between the number of level-1 $T$-type zeroes and level-2 $W$-type zeroes: one is twist-writhe locking relation for A-knotcrystal - the number of level-1 $T$-type zeroes $T_{\left(\mathrm{A}_{1}, \mathrm{~A}_{2}\right), 1 \mathrm{D}}^{I}$ is equal to the number of level-2 $W$-type zeroes $W_{\left(\mathrm{A}_{1}, \mathrm{~A}_{2}\right), 1 \mathrm{D}}^{I}$; The other is twist-writhe locking relation for B-knot-crystal - the number of level-1 T-type zeroes $T_{\left(\mathrm{B}_{1}, \mathrm{~B}_{2}\right), 1 \mathrm{D}}^{I}$ is equal to the number of level-2 $W$-type zeroes $W_{\left(\mathrm{B}_{1}, \mathrm{~B}_{2}\right), 1 \mathrm{D}}^{I}$.

Now, the knot with half linking number on a composite knot-crystal becomes a composite object by trapping half of writhe number (a level-2 $W$-type zero) and half of internal twist number (a level-1 T-type zero), i.e.,

$$
\delta \zeta_{(\mathrm{A}, \mathrm{B}), 1 \mathrm{D}}^{I}=\delta W_{\left(\mathrm{A}_{1}, \mathrm{~A}_{2}\right), 1 \mathrm{D}}^{I}=\delta T_{\left(\mathrm{A}_{1}, \mathrm{~A}_{2}\right), 1 \mathrm{D}}^{I}= \pm \frac{1}{2}
$$

or

$$
\delta \zeta_{(\mathrm{A}, \mathrm{B}), 1 \mathrm{D}}^{I}=\delta W_{\left(\mathrm{B}_{1}, \mathrm{~B}_{2}\right), 1 \mathrm{D}}^{I}=\delta T_{\left(\mathrm{B}_{1}, \mathrm{~B}_{2}\right), 1 \mathrm{D}}^{I}= \pm \frac{1}{2} .
$$

That means a knot traps half of the global winding of the center membranes and half of the internal twisting between two vortex-membranes $A_{1}, A_{2}$ and $B_{1}, B_{2}$. 
According to twist-writhe locking conditions, $\delta W_{\left(\mathrm{A}_{1} \mathrm{~A}_{2}\right), 1 \mathrm{D}}^{I}=-\delta T_{\left(\mathrm{A}_{1} \mathrm{~A}_{2}\right), 1 \mathrm{D}}^{I}=$ $\delta W_{1 \mathrm{D}, \mathrm{A}}^{I}$ and $\delta W_{\left(\mathrm{B}_{1} \mathrm{~B}_{2}\right), 1 \mathrm{D}}^{I}=\delta W_{1 \mathrm{D}, \mathrm{B}}^{I}=-\delta T_{\left(\mathrm{B}_{1} \mathrm{~B}_{2}\right), 1 \mathrm{D}}^{I}$, a global winding of A/B-knotcrystal leads to additional internal twisting between vortex-membranes $A_{1}, A_{2}$ and vortex-membranes $\mathrm{B}_{1}, \mathrm{~B}_{2}$. The quantum phase angle of knot state is the phase angle of $\psi(\vec{x}, t)$ (or the phase angle of $\mathrm{z}_{\mathrm{A} / \mathrm{B}, 2}(\vec{x}, t)$ ) are $\phi(\vec{x}, t)$ (that is $\phi_{\mathrm{A} / \mathrm{B}, 2}(\vec{x}, t)$ ); the phase angle of the internal twisting is $\phi_{\mathrm{A} / \mathrm{B}, 1}(\vec{x}, t)$. Under the phase transformation of knots, according to twist-writhe locking conditions, the phase of internal twisting changes

$$
\Delta \phi_{\mathrm{A} / \mathrm{B}, 2}(\vec{x}, t)+\Delta \phi_{\mathrm{A} / \mathrm{B}, 1}(\vec{x}, t)=0
$$

or

$$
\Delta \phi(\vec{x}, t)+\Delta \phi_{\mathrm{A} / \mathrm{B}, 1}(\vec{x}, t)=0 .
$$

\subsubsection{Emergent $U(1)$ gauge symmetry}

The U(1) gauge symmetry comes from indistinguishable phase of internal twistings inside the composite knot. Based on the hidden information of internal twistings (or internal knots), we show the physics picture of gauge symmetry. It is known that gauge symmetry appears as the redundancy to define the particles. There exists redundancy to define composite knots: the exact initial phase of an internal zero inside a composite knot is not a physical observable value. The $\mathrm{U}(1)$ gauge symmetry indicates that we may locally reorganize the knots by different ways and get the same result. In Fig. 13, we show the theoretical structure of emergent U(1) Abelian gauge symmetry and gauge fields.

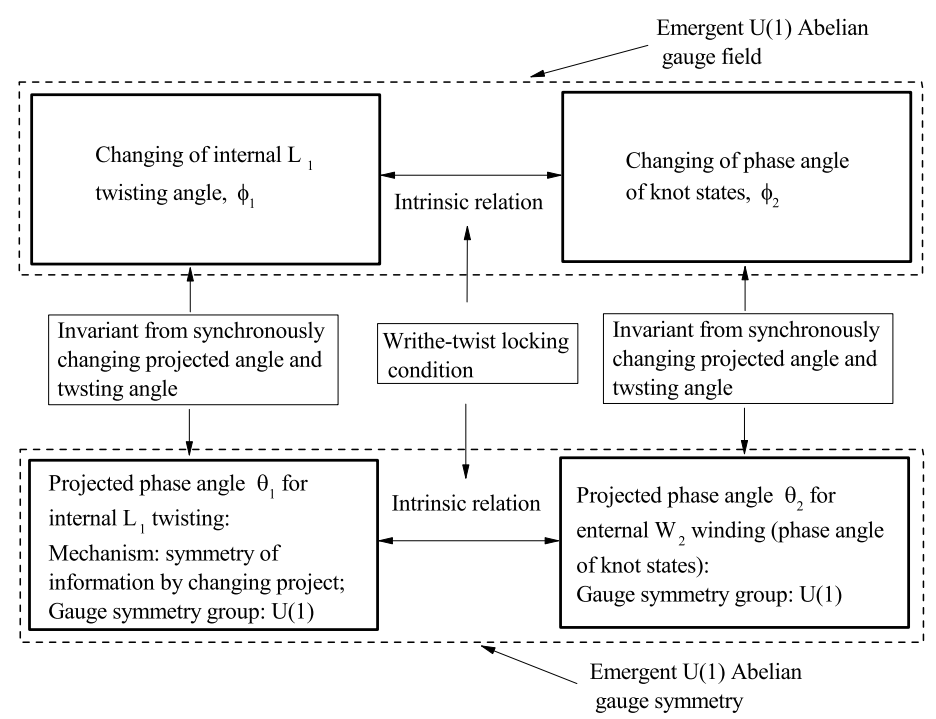

Fig. 13. An illustration of the theoretical structure of emergent $U(1)$ Abelian gauge symmetry and gauge fields. 


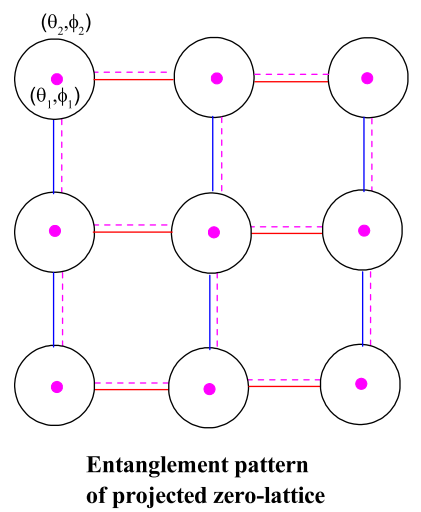

(a)

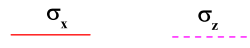

(b)

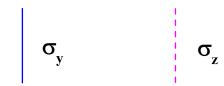

(c)

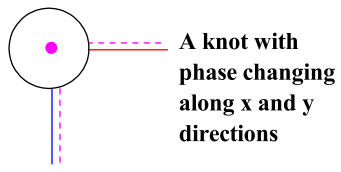

(d)

Fig. 14. (Color online) (a) An illustration of entanglement pattern of a 2D SOC knot-crystal with $(\mathcal{N}=4, \mathcal{M}=2)$ and $\Delta_{(1,2)}=1$. Each circle denotes a level-2 $W$-type zero. The solid lines denote the entanglement pattern for level-1 $T$-type zero-lattice. The dotted lines denote the entanglement pattern for level-1 $T$-type zero-lattice. The purple dots denote internal zeroes; (b) an illustration of entanglement matrices of level-1 and level-2 along $x$-direction; (c) an illustration of entanglement matrices of level-1 and level-2 along $y$-direction; (d) an illustration of a knot.

Let us show the details. To well define a composite knot with an additional $T$-type zero, we must choose a given initial phase angle of internal twistings $\left(\phi_{\mathrm{A} / \mathrm{B}, 1}(\vec{x}, t)\right)_{0}$ on a given point $(\vec{x}, t)$. Owing to twist-writhe locking conditions, the initial phase angle of knot state $(\phi(\vec{x}, t))_{0}=\left(\phi_{\mathrm{A} / \mathrm{B}, 2}(\vec{x}, t)\right)_{0}$ on a given point $(\vec{x}, t)$ is chosen as $(\phi(\vec{x}, t))_{0}=\left(\phi_{\mathrm{A} / \mathrm{B}, 2}(\vec{x}, t)\right)_{0}=\left(\phi_{\mathrm{A} / \mathrm{B}, 1}(\vec{x}, t)\right)_{0}+\phi_{0}$, where $\phi_{0}$ is constant. However, the absolute phase angle of internal twistings is independent on the quantum phase angle of knots. For example, we can set the initial phase angle of internal twistings to $\left(\phi_{\mathrm{A} / \mathrm{B}, 1}(\vec{x}, t)\right)_{0}^{\prime} \neq\left(\phi_{\mathrm{A} / \mathrm{B}, 1}(\vec{x}, t)\right)_{0}$. Different choices of initial phase angle of internal twistings lead to the same physics result. This mechanism leads to the existence of local U(1) gauge symmetry.

Figure 14(a) is an illustration of entanglement pattern of a 2D SOC knot-crystal with $(\mathcal{N}=\overline{4}, \mathcal{M}=2)$ and $\Delta_{(1,2)}=1$. Each circle denotes a level-2 $W$-type zero. The solid lines denote the entanglement pattern for level-1 $T$-type zero-lattice. The dotted lines denote the entanglement pattern for level-1 $T$-type zero-lattice. The purple dots denote internal zeroes. Figure 14(d) is an illustration of a knot.

According to the local U(1) gauge symmetry, the internal states of knots change as the following equation:

$$
|\psi(\vec{x}, t)\rangle \rightarrow\left|\psi^{\prime}(\vec{x}, t)\right\rangle=U_{\mathrm{U}(1)}(\vec{x}, t)|\psi(\vec{x}, t)\rangle .
$$

Due to the local $\mathrm{U}(1)$ gauge symmetry, one cannot distinguish the state $|\psi(\vec{x}, t)\rangle$ with $\left|\psi^{\prime}(\vec{x}, t)\right\rangle=U_{\mathrm{U}(1)}(\vec{x}, t)|\psi(\vec{x}, t)\rangle$. The two knot states $|\psi(\vec{x}, t)\rangle$ and $\left|\psi^{\prime}(\vec{x}, t)\right\rangle=$ $U_{\mathrm{U}(1)}(\vec{x}, t)|\psi(\vec{x}, t)\rangle$ can be the same by changing the initial phase angle of internal twistings. 


\subsubsection{Emergent $\mathrm{U}(1)$ gauge field}

Due to the existence of the local $\mathrm{U}(1)$ gauge symmetry, there exists $\mathrm{U}(1)$ gauge field.

On a composite knot-crystal, the phase of knots are locked to the phase of the internal twisting. Under a local twisting of level-1 $T$-type zero-lattice $\Delta \phi_{\mathrm{A} / \mathrm{B}, 1}(\vec{x}, t)$, there exists corresponding global winding of the level-2 $W$-type zero-lattice

$$
\Delta \phi(\vec{x}, t)=\Delta \phi_{\mathrm{A} / \mathrm{B}, 2}(\vec{x}, t)=-\Delta \phi_{\mathrm{A} / \mathrm{B}, 1}(\vec{x}, t) .
$$

As a result, the phase of the knot at site $\vec{j}$ changes as

$$
\psi_{\vec{j}} \rightarrow e^{i \Delta \phi_{\vec{j}} n_{k}} \psi_{\vec{j}}=e^{-i \Delta \phi_{\vec{j}, \mathrm{~A} / \mathrm{B}, 1} n_{k}} \psi_{\vec{j}} .
$$

Here, the phase-changing $\Delta \phi_{\vec{j}}$ is relative to phase angle of internal twistings $\Delta \phi_{\vec{j}, \mathrm{~A} / \mathrm{B}, 1}$.

After considering the local changing of phase angle induced by additional internal twisting $\Delta \phi_{\vec{j}, \mathrm{~A} / \mathrm{B}, 1}$, the local coupling between two knot states changes, i.e.,

$$
\begin{aligned}
& J \psi_{\vec{j}}^{\dagger} T_{\vec{j}, \vec{j}^{\prime}} \psi_{\vec{j}^{\prime}} \rightarrow J \psi_{\vec{j}}^{\dagger} e^{-i n_{k} \Delta \phi_{\vec{j}}} \cdot T_{\vec{j}, \vec{j}^{\prime}} \cdot e^{i \Delta \phi_{\vec{j}^{\prime}} n_{k}} \psi_{\vec{j}^{\prime}}
\end{aligned}
$$

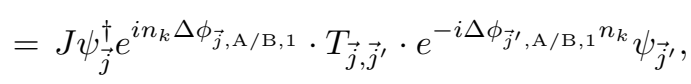

where $T_{\vec{j}, \vec{j}^{\prime}}$ is translation operator from $\vec{j}$-site to $\vec{j}^{\prime}$-site. We define a vector field to characterize the local additional internal twistings $\Delta \phi_{\vec{j}, \mathrm{~A} / \mathrm{B}, 1}$,

$$
A_{\vec{j}, \vec{j}^{\prime}}=\Delta \phi_{\vec{j}, \mathrm{~A} / \mathrm{B}, 1}-\Delta \phi_{\vec{j}^{\prime}, \mathrm{A} / \mathrm{B}, 1}=-\Delta \phi_{\vec{j}}+\Delta \phi_{\vec{j}^{\prime}} .
$$

The local coupling between two knot states becomes

$$
J \psi_{\vec{j}}^{\dagger} T_{\vec{j}, \vec{j}^{\prime}} e^{i e_{0} n_{k} A_{\vec{j}, \vec{j}^{\prime}}} \psi_{\vec{j}^{\prime}}
$$

The total kinetic energy for knots becomes

$$
\hat{\mathcal{H}}_{\text {coupling }}=J \sum_{\left\langle\vec{j}, \vec{j}^{\prime}\right\rangle} \psi_{\vec{j}}^{\dagger} T_{\vec{j}, \vec{j}^{\prime}} e^{i e_{0} n_{k} A_{\vec{j}, \vec{j}^{\prime}}} \psi_{\vec{j}^{\prime}}+\text { h.c. }
$$

It is obvious that the vector field $A_{\vec{j}, \vec{j}^{\prime}}$ that characterizes the local position perturbation of internal zero-lattice plays the role of $\mathrm{U}(1)$ gauge field. To illustrate the local $\mathrm{U}(1)$ gauge symmetry, we do a local $\mathrm{U}(1)$ gauge transformation $U_{\vec{j}, \mathrm{U}(1)}=$ $e^{i \Delta \phi_{0, \vec{j}} n_{k}}$ via changing the initial phase angle of internal twistings

$$
\phi_{0, \vec{j}, \mathrm{~A} / \mathrm{B}, 1} \rightarrow \Delta \phi_{0, \vec{j}, \mathrm{~A} / \mathrm{B}, 1}^{\prime}=\phi_{0, \vec{j}, \mathrm{~A} / \mathrm{B}, 1}+\Delta \phi_{0, \vec{j}, \mathrm{~A} / \mathrm{B}, 1} .
$$

Under above local $\mathrm{U}(1)$ gauge transformation, we have

$$
\psi_{\vec{j}} \rightarrow \psi_{\vec{j}}^{\prime}=U_{\vec{j}, \mathrm{U}(1)} \psi_{\vec{j}}=e^{i \Delta \phi_{0, \vec{j}} n_{k}} \psi_{\vec{j}}
$$

and

$$
e_{0} n_{k} A_{\vec{j}, \vec{j}^{\prime}} \rightarrow e_{0} n_{k} A_{\vec{j}, \vec{j}^{\prime}}^{\prime}=e_{0} n_{k} A_{\vec{j}, \vec{j}^{\prime}}-n_{k}\left(\Delta \phi_{0, \vec{j}, \mathrm{~A} / \mathrm{B}, 1}-\Delta \phi_{0, \vec{j}^{\prime}, \mathrm{A} / \mathrm{B}, 1}\right) .
$$


The total kinetic energy for knots turns into

$$
\hat{\mathcal{H}}_{\text {coupling }} \rightarrow \hat{\mathcal{H}}_{\text {coupling }}^{\prime}=J \sum_{\left\langle\vec{j}, \vec{j}^{\prime}\right\rangle} \psi_{\vec{j}}^{\prime \dagger} T_{\vec{j}, \vec{j}^{\prime}} e^{i e_{0} n_{k} A_{\vec{j}, \vec{j}^{\prime}}^{\prime}} \psi_{\vec{j}^{\prime}}^{\prime}+\text { h.c. }
$$

According to the twist-writhe locking condition $\phi_{\vec{j}, \mathrm{~A} / \mathrm{B}, 1}=-\Delta \phi_{\vec{j}}$, the Hamiltonian does not change,

$$
\hat{\mathcal{H}}_{\text {coupling }}=\hat{\mathcal{H}}_{\text {coupling }}^{\prime} \text {. }
$$

On the other hand, the situation for tempo phase-changing is similar to that for spatial phase-changing. To characterize the tempo twist-writhe locking condition, we introduce a Lagrangian variable $A_{0, \vec{j}}$ to path integral formulation as

$$
A_{0, \vec{j}} \psi_{\vec{j}}^{\dagger} \psi_{\vec{j}}
$$

In continuum limit, we have $U_{\vec{j}, \mathrm{U}(1)}(t) \rightarrow U_{\mathrm{U}(1)}(\vec{x}, t), A_{\vec{j}, \vec{j}^{\prime}} \rightarrow \vec{A}(x)$ and $A_{0, \vec{j}} \rightarrow$ $A_{0}(x)$. The Abelian gauge symmetry is represented by

$$
\psi^{\prime} \rightarrow U_{\mathrm{SU}(n)}(\vec{x}, t) \psi
$$

and

$$
\begin{aligned}
A_{\mu}(\vec{x}, t) & \rightarrow A_{\mu}(\vec{x}, t)+i\left(\partial_{\mu} U_{\mathrm{U}(1)}(\vec{x}, t)\right)\left(U_{\mathrm{U}(1)}(\vec{x}, t)\right)^{-1} \\
& =A_{\mu}(\vec{x}, t)+\frac{1}{e_{0} n_{k}} \partial_{\mu} \phi(\vec{x}, t) .
\end{aligned}
$$

Finally, we derive the path integral formulation to characterize the effective Hamiltonian in continuum limit for gauge field

$$
\int \mathcal{D} A_{0} \mathcal{D} \vec{A} e^{i \mathcal{S}_{\mathrm{EM}} / \hbar}
$$

where $\mathcal{S}_{\mathrm{EM}}=\int \mathcal{L}_{\mathrm{EM}} d t d^{3} x$ and

$$
\mathcal{L}_{\mathrm{EM}}=\mathrm{e} A_{\mu} j_{(e m)}^{\mu}-\frac{1}{4} F_{\mu \nu} F^{\mu \nu} .
$$

The gauge field strength $F_{\mu \nu}$ is defined by $F_{\mu \nu}=\partial_{\mu} A_{\nu}-\partial_{\nu} A_{\mu}$. The electric charge for an internal twisting zero is $e_{0}$. As a result, the total electric charge of a composite knot with $n$-internal zeroes is just $\mathrm{e}=n e_{0}$. It is obvious that $\mathcal{L}_{\mathrm{EM}}$ has local $\mathrm{U}(1)$ gauge symmetry. On the contrary, people always obtain the above formula $\left(\mathcal{L}_{\mathrm{EM}}=\right.$ $\left.\mathrm{e} A_{\mu} j_{(e m)}^{\mu}-\frac{1}{4} F_{\mu \nu} F^{\mu \nu}\right)$ from the point of view of local gauge symmetry.

From Eq. (239), we can derive the Maxwell equations

$$
\partial_{\mu} F^{\mu \nu}=j_{(e m)}^{\mu}
$$

where $j_{(e m)}^{\mu}$ is the electric current. In addition, to give a correct definition of knots in a composite knot-crystal, we must set down the "gauge" $f\left(A_{\mu}\right)=0$ that leads to a fixed $\phi_{0, \vec{j}, \mathrm{~A} / \mathrm{B}, 1}(\vec{x}, t)$ at a give position $(\vec{x}, t)$ for internal twisting.

For the case of $n=1$, we derive the U(1) gauge theory for composite knotcrystal. Now,the charge of an electron is $e_{0}$. As a result, the effective Lagrangian 
for knots turns into

$$
\mathcal{L}_{\text {fermion }}(x)=\bar{e}(x) i \gamma^{\mu} \partial_{\mu} e(x)+m_{e} \bar{e}(x) e(x)+e_{0} A_{\mu}(x) j_{(e m)}^{\mu}(x),
$$

where

$$
j_{(e m)}^{\mu}(x)=i \bar{e}(x) \gamma^{\mu} e(x) .
$$

For the case of $n=2$, the charge of an electron is $2 e_{0}$; the charge of a quark is $n_{\text {quark }} \cdot e_{0}=e_{0}$. As a result, the effective Lagrangian for knots turns into

$$
\begin{aligned}
\mathcal{L}_{\text {fermion }}(x)= & \bar{e}(x) i \gamma^{\mu} \partial_{\mu} e(x)+\bar{q}(x) i \gamma^{\mu} \partial_{\mu} q(x) \\
& +m_{e} \bar{e}(x) e(x)+m_{q} \bar{q}(x) q(x)+\mathrm{e} A_{\mu}(x) j_{(e m)}^{\mu}(x),
\end{aligned}
$$

where

$$
j_{(e m)}^{\mu}(x)=i \bar{e}(x) \gamma^{\mu} e(x)+i \frac{1}{2} \bar{q}(x) \gamma^{\mu} q(x) .
$$

For the case of $n=3$, the charge of an electron is $3 e_{0}$; the charge of a $u$-quark is $n_{u \text {-quark }} \cdot e_{0}=2 e_{0}$, the charge of a $d$-quark is $n_{d \text {-quark }}=e_{0}$. As a result, the effective Lagrangian for knots turns into

$$
\begin{aligned}
\mathcal{L}_{\text {fermion }}(x)= & \bar{e}(x) i \gamma^{\mu} \partial_{\mu} e(x)+\bar{d}(x) i \gamma^{\mu} \partial_{\mu} d(x)+\bar{u}(x) i \gamma^{\mu} \partial_{\mu} u(x)+m_{e} \bar{e}(x) e(x) \\
& +m_{d} \bar{d}(x) d(x)+m_{u} \bar{u}(x) u(x)+\mathrm{e} A_{\mu}(x) j_{(\mathrm{em})}^{\mu}(x)
\end{aligned}
$$

where

$$
j_{(e m)}^{\mu}(x)=i \bar{e}(x) \gamma^{\mu} e(x)+i \frac{2}{3} \bar{u}(x) \gamma^{\mu} u(x)+i \frac{1}{3} \bar{d}(x) \gamma^{\mu} d(x)
$$

Here, we have set the charge of an electron $3 e_{0}$ to be e.

Finally, we show the physical picture of the U(1) gauge field. U(1) gauge field comes from phase fluctuations of internal zero-lattice. Two composite knots interact by exchanging phase fluctuations of internal zero-lattice. An extra internal zero plays the role of the source of electric field. When there exists an extra internalwinding, the composite knots will be attractive or repulsive to the source. When there exists finite magnetic field, the path of moving composite knots will be curved. A photon is a local internal twist changing. Because the longitudinal fluctuation of internal twisting is "eaten" knots due to writhe-twist locking condition, the fluctuations of internal twisting have only transverse modes. This is why the gauge fields are transverse waves with spin-1.

In addition, we compare the physics picture of U(1) gauge symmetry in composite knot-crystal and that in Kaluza-Klein theory. ${ }^{23}$ In knot physics, each internal zero corresponds to a $\pi$-flux in extra space $\left(x_{d+1}-x_{d+2}\right.$ space) and the deformation of internal zero-lattice becomes electromagnetic field, the fluctuations of internal zero-lattice becomes Maxwell waves. For composite knot with a internal zero, the total flux in extra space is $\pi$, of which the total electric charge is $e_{0}$. The situation is very similar to the emergent gauge symmetry in Kaluza-Klein theory, in which the particles have finite angular momentum $m$ in extra space that corresponds to a half internal twisting (a internal zero) in knot physics. 


\subsection{Emergent $\mathrm{SU}(n)$ gauge field}

\subsubsection{Emergent $S U(n)$ gauge symmetry}

In this part, we discuss the emergent quantum field theory for composite knots with taking into account the dynamics of internal zeroes/knots. For a 2-level composite knot-crystal with $(\mathcal{N}=4, \mathcal{M}=2)$ and an integer hierarchy number $\Delta_{(1,2)}=n$, there are $n$ different types of knots with $[1,0],[1,1], \ldots[1, n-1]$. The composite knots with $[1, n]$ correspond to electrons in particle physics and the composite knots with $\left[1, n_{k}\right]\left(n_{k}\right.$ is an integer number, $\left.0<n_{k}<n\right)$ correspond to quarks. We point out that an emergent $\mathrm{SU}(n)$ non-Abelian gauge field describes the knot dynamics on a composite knot-crystal. The SU( $n)$ gauge symmetry comes from the indistinguishable states of internal-knots inside the composite knot. In Fig. 15, we show the theoretical structure of emergent $\mathrm{SU}(n)$ non-Abelian gauge symmetry and non-Abelian gauge fields.

For the knot-crystal, each level-2 type- $T$ zero (for example, a knot at $(\vec{x}, t)$ ) corresponds to $n$ level-1 internal type- $T$ zeroes. Because each internal zero corresponds to an internal knot, we label the $n$ internal zeroes by $1,2, \ldots, n$ and the corresponding states of internal knots by $\left|\psi_{\text {inter, } 1}\right\rangle,\left|\psi_{\text {inter }, 2}\right\rangle, \ldots,\left|\psi_{\text {inter }, n}\right\rangle$, respectively. $\left|\psi_{\text {inter }, 1}\right\rangle,\left|\psi_{\text {inter }, 2}\right\rangle, \ldots,\left|\psi_{\text {inter }, n}\right\rangle$ have the same properties and $\left|\psi_{\text {inter, } 1}\right\rangle,\left|\psi_{\text {inter }, 2}\right\rangle, \ldots,\left|\psi_{\text {inter, } n}\right\rangle$ make up a complete basis. In general, due to symmetry of $\left|\psi_{\text {inter }, 1}\right\rangle,\left|\psi_{\text {inter, } 2}\right\rangle, \ldots,\left|\psi_{\text {inter }, n}\right\rangle$, we can relabel the corresponding states of internal knots by $\left|\psi_{\text {inter }, 1}^{\prime}\right\rangle,\left|\psi_{\text {inter }, 2}^{\prime}\right\rangle, \ldots,\left|\psi_{\text {inter }, n}^{\prime}\right\rangle$. The relationship between the

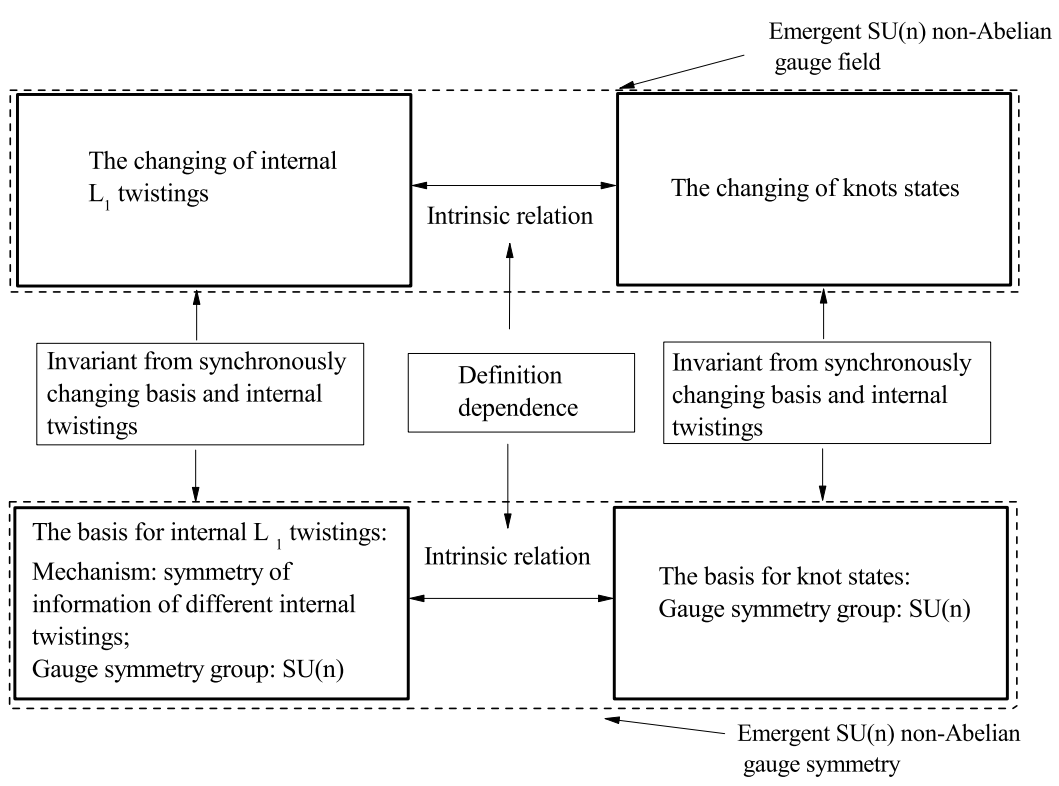

Fig. 15. An illustration of the theoretical structure of emergent $\mathrm{SU}(n)$ non-Abelian gauge symmetry and non-Abelian gauge fields. 
two bases is

$$
\left(\begin{array}{c}
\left|\psi_{\text {inter }, 1}^{\prime}(\vec{x}, t)\right\rangle \\
\left|\psi_{\text {inter }, 2}^{\prime}(\vec{x}, t)\right\rangle \\
\ldots \\
\left|\psi_{\text {inter }, n}^{\prime}(\vec{x}, t)\right\rangle
\end{array}\right)=U_{\mathrm{SU}(n)}(\vec{x}, t)\left(\begin{array}{c}
\left|\psi_{\text {inter }, 1}\right\rangle \\
\left|\psi_{\text {inter }, 2}\right\rangle \\
\ldots \\
\left|\psi_{\text {inter }, n}\right\rangle
\end{array}\right),
$$

where $U_{\mathrm{SU}(n)}(\vec{x}, t)=e^{i \Theta(\vec{x}, t)}$ is the matrix of the representation of $\mathrm{SU}(n)$ group. $\Theta(\vec{x}, t)=\sum_{a=1}^{n^{2}-1} \theta^{a}(\vec{x}, t) \tau^{a}$ and $\theta^{a}$ are a set of $n^{2}-1$ constant parameters, and $\tau^{a}$ are $n^{2}-1 n \times n$ matrices representing the $n^{2}-1$ generators of the Lie algebra of $\mathrm{SU}(n) \cdot{ }^{19}$ This result leads to the concept of non-Abelian gauge symmetry.

Thus, we have a local $\mathrm{SU}(n)$ symmetry that denotes the indistinguishable states of internal-knots inside the composite knot.

For the case of $n=2$, there are two internal zeroes inside a knot that is labeled by 1,2 . The corresponding basis of states of internal knots is $\left(\begin{array}{l}\left|\psi_{\text {inter }, 1}(\vec{x}, t)\right\rangle \\ \left|\psi_{\text {inter }, 2}(\vec{x}, t)\right\rangle\end{array}\right)$. Due to the local SU(2) gauge symmetry, the basis can be arbitrarily redefined, i.e.,

$$
\left(\begin{array}{c}
\left|\psi_{\text {inter }, 1}(\vec{x}, t)\right\rangle \\
\left|\psi_{\text {inter }, 2}(\vec{x}, t)\right\rangle
\end{array}\right) \rightarrow\left(\begin{array}{c}
\left|\psi_{\text {inter }, 1}^{\prime}(\vec{x}, t)\right\rangle \\
\left|\psi_{\text {inter }, 2}^{\prime}(\vec{x}, t)\right\rangle
\end{array}\right)=U_{\mathrm{SU}(2)}(\vec{x}, t)\left(\begin{array}{l}
\left|\psi_{\text {inter }, 1}(\vec{x}, t)\right\rangle \\
\left|\psi_{\text {inter }, 2}(\vec{x}, t)\right\rangle
\end{array}\right)
$$

where $U_{\mathrm{SU}(2)}(\vec{x}, t)=e^{i \Theta(\vec{x}, t)}$ is the matrix of the representation of $\mathrm{SU}(2)$ group. $\Theta(\vec{x}, t)=\sum_{a=1}^{3} \theta^{a}(\vec{x}, t) \tau^{a}$ and $\theta^{a}$ are a set of three constant parameters, and $\tau^{a}$ are $2 \times 2$ Pauli matrices representing the three generators of the Lie algebra of SU(2). .9

When there exists a knot with $[1,1]$, the knot states are denoted by

$$
\left(\begin{array}{c}
\left|q_{1}(\vec{x}, t)\right\rangle \\
\left|q_{2}(\vec{x}, t)\right\rangle
\end{array}\right)=\left(\begin{array}{c}
q^{\dagger}(\vec{x}, t)\left|\psi_{\text {inter }, 1}(\vec{x}, t)\right\rangle \\
q^{\dagger}(\vec{x}, t)\left|\psi_{\text {inter }, 2}(\vec{x}, t)\right\rangle
\end{array}\right)=\left(\begin{array}{c}
q_{1}^{\dagger}(\vec{x}, t)|\mathrm{vac}\rangle \\
q_{2}^{\dagger}(\vec{x}, t)|\operatorname{vac}\rangle
\end{array}\right)
$$

where $\left|q_{1}(\vec{x}, t)\right\rangle$ denotes a knot state with an internal knot of 1st internal-zero and $\left|q_{2}(\vec{x}, t)\right\rangle$ denotes a knot state with an internal knot of 2 nd internal-zero. $q^{\dagger}(\vec{x}, t)$ is the creation operator of level-2 knot with two internal knot states. Because both the internal knot state $\left|q_{1}(\vec{x}, t)\right\rangle$ and the internal knot state $\left|q_{2}(\vec{x}, t)\right\rangle$ change level-1 half linking number, we cannot distinguish the knots with the two different internal

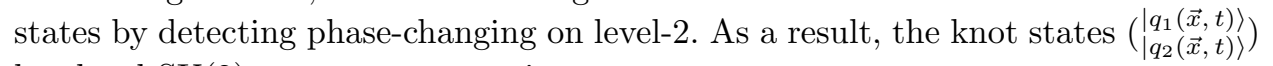
has local $\mathrm{SU}(2)$ gauge symmetry, i.e.,

$$
\begin{aligned}
& \left(\begin{array}{c}
\left|q_{1}(\vec{x}, t)\right\rangle \\
\left|q_{2}(\vec{x}, t)\right\rangle
\end{array}\right) \rightarrow\left(\begin{array}{c}
\left|q_{1}^{\prime}(\vec{x}, t)\right\rangle \\
\left|q_{2}^{\prime}(\vec{x}, t)\right\rangle
\end{array}\right) \\
& \quad=\left(\begin{array}{c}
q^{\dagger}(\vec{x}, t)\left|\psi_{\text {inter }, 1}^{\prime}(\vec{x}, t)\right\rangle \\
q^{\dagger}(\vec{x}, t)\left|\psi_{\text {inter }, 2}^{\prime}(\vec{x}, t)\right\rangle
\end{array}\right) \\
& =\left(\begin{array}{l}
q^{\dagger}(\vec{x}, t) U_{\mathrm{SU}(2)}(\vec{x}, t)\left|\psi_{\text {inter }, 1}(\vec{x}, t)\right\rangle \\
q^{\dagger}(\vec{x}, t) U_{\mathrm{SU}(2)}(\vec{x}, t)\left|\psi_{\text {inter }, 2}(\vec{x}, t)\right\rangle
\end{array}\right)
\end{aligned}
$$




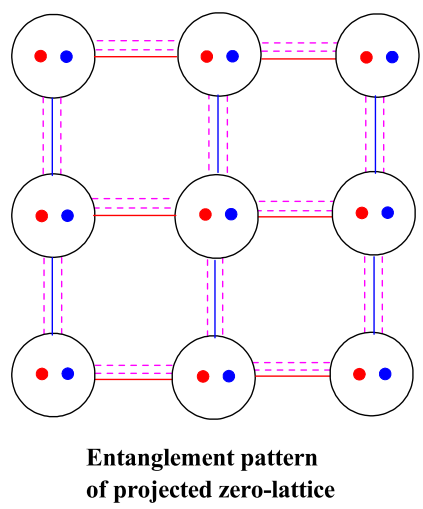

(a)

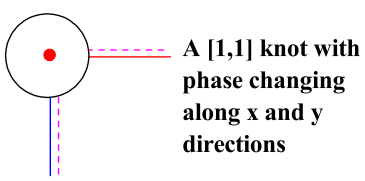

(b)

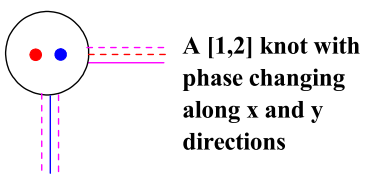

(c)

Fig. 16. (Color online) (a) An illustration of entanglement pattern of a 2D SOC knot-crystal with $(\mathcal{N}=4, \mathcal{M}=2)$ and $\Delta_{(1,2)}=2$. Each circle denotes a level-2 $W$-type zero. The solid lines denote the entanglement pattern for level-1 $T$-type zero-lattice. The dotted lines denote the entanglement pattern for level-1 $T$-type zero-lattice. The dots inside the circles denote internal zeroes; (b) an illustration of a [1,1] knot; (c) an illustration of a [1,2] knot.

$$
\begin{aligned}
& =U_{\mathrm{SU}(2)}(\vec{x}, t)\left(\begin{array}{l}
q^{\dagger}(\vec{x}, t)\left|\psi_{\text {inter }, 1}(\vec{x}, t)\right\rangle \\
q^{\dagger}(\vec{x}, t)\left|\psi_{\text {inter }, 2}(\vec{x}, t)\right\rangle
\end{array}\right) \\
& =U_{\mathrm{SU}(2)}(\vec{x}, t)\left(\begin{array}{l}
\left|q_{1}(\vec{x}, t)\right\rangle \\
\left|q_{2}(\vec{x}, t)\right\rangle
\end{array}\right) .
\end{aligned}
$$

Therefore, the quarks also have local $\mathrm{SU}(2)$ gauge symmetry. This leads to the existence of $\mathrm{SU}(2)$ non-Abelian gauge fields.

Figure 16(a) is an illustration of entanglement pattern of a 2D SOC knot-crystal with $(\mathcal{N}=4, \mathcal{M}=2)$ and $\Delta_{(1,2)}=2$. Each circle denotes a level-2 $W$-type zero. The solid lines denote the entanglement pattern for level-1 $T$-type zero-lattice. The dotted lines denote the entanglement pattern for level-1 $T$-type zero-lattice. The dots inside the circles denote internal zeroes. Figures 16(b) and 16(c) illustrate $[1,1]$ knot and $[1,2]$ knot, respectively.

According to the local SU(2) gauge symmetry, the internal states of quarks change as the following equation:

$$
\left(\begin{array}{l}
\left|q_{1}(\vec{x}, t)\right\rangle \\
\left|q_{2}(\vec{x}, t)\right\rangle
\end{array}\right) \rightarrow\left(\begin{array}{l}
\left|q_{1}^{\prime}(\vec{x}, t)\right\rangle \\
\left|q_{2}^{\prime}(\vec{x}, t)\right\rangle
\end{array}\right)=U_{\mathrm{SU}(2)}(\vec{x}, t)\left(\begin{array}{l}
\left|q_{1}(\vec{x}, t)\right\rangle \\
\left|q_{2}(\vec{x}, t)\right\rangle
\end{array}\right) .
$$

Due to the local SU(2) gauge symmetry, one cannot distinguish the state $\left(\begin{array}{l}\left|q_{1}(\vec{x}, t)\right\rangle \\ \left|q_{2}(\vec{x}, t)\right\rangle\end{array}\right)$ with

$$
\left(\begin{array}{l}
\left|q_{1}^{\prime}(\vec{x}, t)\right\rangle \\
\left|q_{2}^{\prime}(\vec{x}, t)\right\rangle
\end{array}\right)=U_{\mathrm{SU}(2)}(\vec{x}, t)\left(\begin{array}{l}
\left|q_{1}(\vec{x}, t)\right\rangle \\
\left|q_{2}(\vec{x}, t)\right\rangle
\end{array}\right)
$$


The knot states $\left(\begin{array}{l}\left|q_{1}(\vec{x}, t)\right\rangle \\ \left|q_{2}(\vec{x}, t)\right\rangle\end{array}\right)$ and the knot states

$$
\left(\begin{array}{l}
\left|q_{1}^{\prime}(\vec{x}, t)\right\rangle \\
\left|q_{2}^{\prime}(\vec{x}, t)\right\rangle
\end{array}\right)=U_{\mathrm{SU}(2)}(\vec{x}, t)\left(\begin{array}{l}
\left|q_{1}(\vec{x}, t)\right\rangle \\
\left|q_{2}(\vec{x}, t)\right\rangle
\end{array}\right)
$$

can be the same by changing the basis of states of internal knots.

For the case of $n=3$, there are three internal zeroes inside a composite knot that is labeled by $1,2,3$. The basis of states of internal knots has local $\mathrm{SU}(3)$ gauge symmetry

$$
\left(\begin{array}{l}
\left|\psi_{\text {inter }, 1}(\vec{x}, t)\right\rangle \\
\left|\psi_{\text {inter }, 2}(\vec{x}, t)\right\rangle \\
\left|\psi_{\text {inter }, 3}(\vec{x}, t)\right\rangle
\end{array}\right) \text {. }
$$

After reordering the internal zeroes, the states of $d$-quarks

$$
\left(\begin{array}{l}
d_{1, \tau, \sigma}|\mathrm{vac}\rangle \\
d_{2, \tau, \sigma}|\mathrm{vac}\rangle \\
d_{3, \tau, \sigma}|\mathrm{vac}\rangle
\end{array}\right)
$$

and those of $u$-quarks

$$
\left(\begin{array}{l}
u_{1, \tau, \sigma}|\mathrm{vac}\rangle \\
u_{2, \tau, \sigma}|\mathrm{vac}\rangle \\
u_{3, \tau, \sigma}|\mathrm{vac}\rangle
\end{array}\right)
$$

also have local SU(3) gauge symmetry, i.e.,

$$
\begin{aligned}
& \left(\begin{array}{l}
d_{1, \tau, \sigma}|\mathrm{vac}\rangle \\
d_{2, \tau, \sigma}|\mathrm{vac}\rangle \\
d_{3, \tau, \sigma}|\mathrm{vac}\rangle
\end{array}\right) \rightarrow\left(\begin{array}{l}
d_{1, \tau, \sigma}^{\prime}|\mathrm{vac}\rangle \\
d_{2, \tau, \sigma}^{\prime}|\mathrm{vac}\rangle \\
d_{3, \tau, \sigma}^{\prime}|\mathrm{vac}\rangle
\end{array}\right)=U_{\mathrm{SU}(3)}(\vec{x}, t)\left(\begin{array}{l}
d_{1, \tau, \sigma}|\mathrm{vac}\rangle \\
d_{2, \tau, \sigma}|\mathrm{vac}\rangle \\
d_{3, \tau, \sigma}|\mathrm{vac}\rangle
\end{array}\right), \\
& \left(\begin{array}{l}
u_{1, \tau, \sigma}|\mathrm{vac}\rangle \\
u_{2, \tau, \sigma}|\mathrm{vac}\rangle \\
u_{3, \tau, \sigma}|\mathrm{vac}\rangle
\end{array}\right) \rightarrow\left(\begin{array}{l}
u_{1, \tau, \sigma}^{\prime}|\mathrm{vac}\rangle \\
u_{2, \tau, \sigma}^{\prime}|\mathrm{vac}\rangle \\
u_{3, \tau, \sigma}^{\prime}|\mathrm{vac}\rangle
\end{array}\right)=U_{\mathrm{SU}(3)}(\vec{x}, t)\left(\begin{array}{l}
u_{1, \tau, \sigma}|\mathrm{vac}\rangle \\
u_{2, \tau, \sigma}|\mathrm{vac}\rangle \\
u_{3, \tau, \sigma}|\mathrm{vac}\rangle
\end{array}\right) .
\end{aligned}
$$

Here $U_{\mathrm{SU}(3)}(\vec{x}, t)=e^{i \Theta(\vec{x}, t)}$ is the matrix of the representation of $\mathrm{SU}(3)$ group. $\Theta(\vec{x}, t)=\sum_{a=1}^{8} \theta^{a}(\vec{x}, t) \tau^{a}$ and $\theta^{a}$ are a set of eight constant parameters, and $\tau^{a}$ are eight $3 \times 3$ matrices representing the eight generators of the Lie algebra of SU(3). 19

Figure 17 is an illustration of entanglement pattern of a 2D SOC knot-crystal with $(\mathcal{N}=\overline{4}, \mathcal{M}=2)$ and $\Delta_{(1,2)}=3$. Each circle denotes a level-2 $W$-type zero. The solid lines denote the entanglement pattern for level-1 $T$-type zero-lattice. The dotted lines denote the entanglement pattern for level-1 $T$-type zero-lattice. The 


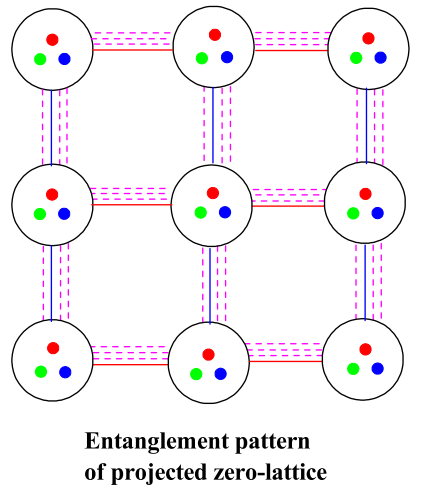

(a)

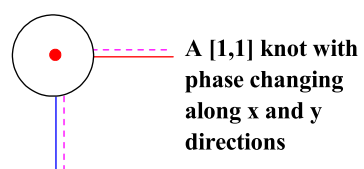

(b)

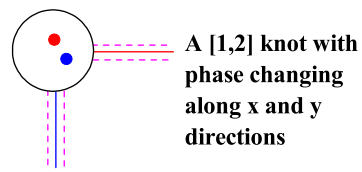

(c)

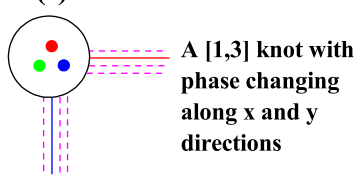

(d)

Fig. 17. (Color online) (a) An illustration of entanglement pattern of a 2D SOC knot-crystal with $(\mathcal{N}=4, \mathcal{M}=2)$ and $\Delta_{(1,2)}=3$. Each circle denotes a level-2 $W$-type zero. The solid lines denote the entanglement pattern for level-1 $T$-type zero-lattice. The dotted lines denote the entanglement pattern for level-1 $T$-type zero-lattice. The dots inside the circles denote internal zeroes; (b) an illustration of a [1,1] knot; (c) an illustration of a [1,2] knot; (d) an illustration of a $[1,3]$ knot.

dots inside the circles denote internal zeroes; Figs. 17(b)-17(d) illustrate $[1,1]$ knot, $[1,2]$ knot, $[1,3]$ knot, respectively.

We then derive the formulation of the gauge theory for the quarks with local $\mathrm{SU}(n)$ symmetry. For simplicity, we denote the basis of quarks with $n$ internal knot states by

$$
\Psi_{\text {quark }}=\left(\begin{array}{c}
\psi_{1, \text { quark }} \\
\psi_{2, \text { quark }} \\
\ldots \\
\psi_{n, \text { quark }}
\end{array}\right),
$$

where $\psi_{i \text {,quark }}$ describes the quantum state of the composite knots. Due to local $\mathrm{SU}(n)$ symmetry of internal states for internal-knots, $\psi_{i \text {,quark }}$ and $\psi_{i^{\prime}}$,quark can be changed into each other by choosing different local gauges. Thus, $\Psi_{\text {quark }}$ can be transformed locally according to an $n$-dimensional representation,

$$
\Psi_{\text {quark }} \rightarrow \Psi_{\text {quark }}^{\prime}=U_{\mathrm{SU}(n)}(x) \Psi_{\text {quark }},
$$

where $U_{\mathrm{SU}(n)}(x)=e^{i \Theta(x)}$ is the matrix of the representation of $\mathrm{SU}(n)$ group.

\subsubsection{Emergent $\mathrm{SU}(n)$ gauge theory for composite knot-crystal}

The local symmetry of internal states $\left(U_{\mathrm{SU}(n)}(\vec{x}, t)|\psi\rangle=|\psi\rangle^{\prime}\right)$ for internal-knots leads to an $\mathrm{SU}(n)$ gauge symmetry for composite knots (elementary fermions). 
The $\mathrm{SU}(n)$ gauge symmetry for composite knots (elementary fermions) leads to emergent $\mathrm{SU}(n)$ gauge theory for composite knot-crystal.

To well define a composite knot with $n$ internal zeroes, we must choose arbitrary $n$ nearest-neighbor zeroes. Gauge symmetries appear as the redundancies to choose which $n$ internal zeroes make up this composite knot. The redundancy protected by internal symmetry for $n$ internal zeroes leads to a non-Abelian gauge symmetry that is really the $\mathrm{SU}(n)$ gauge symmetry for strong interaction. As a result, we have an $\mathrm{SU}(n)$ gauge symmetry that will never be broken.

Due to the existence of the local $\mathrm{SU}(n)$ gauge symmetry, there exists $\mathrm{SU}(n)$ gauge field. Under changing internal knots inside a composite knot, the quark state at site $\vec{j}$ changes as

$$
q_{\vec{j}} \rightarrow q_{\vec{j}}^{\prime}=\tilde{U}_{\vec{j}, \mathrm{SU}(n)} q_{\vec{j}} .
$$

Here, $\tilde{U}_{\mathrm{SU}(n)}$ is a changing of internal knot states based on certain basis

$$
\left(\begin{array}{c}
\psi_{1, \text { quark }} \\
\psi_{2, \text { quark }} \\
\cdots \\
\psi_{n, \text { quark }}
\end{array}\right) .
$$

After considering the local changing of basis induced by $U_{\vec{j}, \mathrm{SU}(n)}$, the local coupling between two quark states changes, i.e.,

$$
J q_{\vec{j}}^{\dagger} T_{\vec{j}, \vec{j}^{\prime}} q_{\vec{j}^{\prime}} \rightarrow J\left(q_{\vec{j}} \tilde{U}_{\vec{j}, \mathrm{SU}(n)}\right)^{\dagger} \cdot T_{\vec{j}, \vec{j}^{\prime}} \cdot\left(\tilde{U}_{\vec{j}^{\prime}, \mathrm{SU}(n)} q_{\vec{j}^{\prime}}\right),
$$

where $T_{\vec{j}, \vec{j}^{\prime}}$ is translation operator from $\vec{j}$-site to $\vec{j}^{\prime}$-site. We define a vector field $\mathcal{A}_{\vec{j}, \vec{j}^{\prime}}$ to characterize the local changing of basis

$$
e^{i g \mathcal{A}_{\vec{j}, \vec{j}^{\prime}}}=\left(\tilde{U}_{\vec{j}, \mathrm{SU}(n)}\right)^{-1} \tilde{U}_{\vec{j}^{\prime}, \mathrm{SU}(n)},
$$

where $g$ is the coupling constant of $\mathrm{SU}(n)$ non-Abelian gauge field. So, for perturbation case $\mathcal{A}_{\vec{j}, \vec{j}^{\prime}} \sim 0$, we have

$$
i g \mathcal{A}_{\vec{j}, \vec{j}^{\prime}} \simeq\left(\delta \tilde{U}_{\vec{j}, \mathrm{SU}(n)}^{-1}\right) \tilde{U}_{\vec{j}^{\prime}, \mathrm{SU}(n)}
$$

where $\delta \tilde{U}_{\vec{j}, \mathrm{SU}(n)}^{-1}=\tilde{U}_{\vec{j}, \mathrm{SU}(n)}^{-1}-\tilde{U}_{\vec{j}^{\prime}, \mathrm{SU}(n)}^{-1}$. The local coupling between two knot states becomes

$$
J q_{\vec{j}}^{\dagger} e^{i g \mathcal{A}_{\vec{j}, \vec{j}^{\prime}}} T_{\vec{j}, \vec{j}^{\prime}} q_{\vec{j}^{\prime}}
$$

The total kinetic energy for knots becomes

$$
\hat{\mathcal{H}}_{\text {coupling }}=J \sum_{\left\langle\vec{j}, \vec{j}^{\prime}\right\rangle} J q_{\vec{j}}^{\dagger} e^{i g \mathcal{A}_{\vec{j}, \vec{j}^{\prime}}} T_{\vec{j}, \vec{j}^{\prime}} q_{\vec{j}^{\prime}}+\text { h.c. }
$$

It is obvious that the vector field $\mathcal{A}_{\vec{j}, \vec{j}^{\prime}}$ that characterizes the local position perturbation of internal zero-lattice plays the role of $\mathrm{SU}(n)$ gauge field. To illustrate 
the local $\mathrm{SU}(n)$ gauge symmetry, we do a local $\mathrm{SU}(n)$ gauge transformation $U_{\vec{j}, \mathrm{SU}(n)}$ that is the transformation of basis of internal knot states, i.e.,

$$
\left(\begin{array}{c}
\psi_{1, \text { quark }}^{\prime} \\
\psi_{2, \text { quark }}^{\prime} \\
\cdots \\
\psi_{n, \text { quark }}^{\prime}
\end{array}\right)=U_{\vec{j}, \mathrm{SU}(n)}\left(\begin{array}{c}
\psi_{1, \text { quark }} \\
\psi_{2, \text { quark }} \\
\cdots \\
\psi_{n, \text { quark }}
\end{array}\right) .
$$

Under a local $\mathrm{SU}(n)$ gauge transformation, we have

$$
q_{\vec{j}} \rightarrow q_{\vec{j}}^{\prime}=U_{\vec{j}, \mathrm{SU}(n)} q_{\vec{j}}
$$

and

$$
g \mathcal{A}_{\vec{j}, \vec{j}^{\prime}} \rightarrow g \mathcal{A}_{\vec{j}, \vec{j}^{\prime}}^{\prime}=g U_{\vec{j}, \mathrm{SU}(n)} \mathcal{A}_{\vec{j}, \vec{j}^{\prime}}\left(U_{\vec{j}^{\prime}, \mathrm{SU}(n)}\right)^{-1}+i\left(\delta U_{\vec{j}, \mathrm{SU}(n)}^{-1}\right)\left(U_{\vec{j}, \mathrm{SU}(n)}\right)^{-1},
$$

where $\delta U_{\vec{j}, \mathrm{SU}(n)}^{-1}=\left(U_{\vec{j}, \mathrm{SU}(n)}^{-1}-U_{\vec{j}^{\prime}, \mathrm{SU}(n)}^{-1}\right)$. Here, we have used the following result:

$$
\begin{aligned}
e^{i g \mathcal{A}_{\vec{j}, \vec{j}^{\prime}}} & =\left(\tilde{U}_{\vec{j}, \mathrm{SU}(n)}\right)^{-1} \tilde{U}_{\vec{j}^{\prime}, \mathrm{SU}(n)} \rightarrow e^{i g \mathcal{A}_{\vec{j}, \vec{j}^{\prime}}^{\prime}} \\
& =U_{\vec{j}, \mathrm{SU}(n)} e^{i g \mathcal{A}_{\vec{j}, \vec{j}^{\prime}}\left(U_{\vec{j}^{\prime}, \mathrm{SU}(n)}\right)^{-1}} \\
& =U_{\vec{j}, \mathrm{SU}(n)}\left(1+i g \mathcal{A}_{\vec{j}, \vec{j}^{\prime}}\right)\left(U_{\vec{j}^{\prime}, \mathrm{SU}(n)}\right)^{-1} \\
& =U_{\vec{j}, \mathrm{SU}(n)}\left(U_{\vec{j}^{\prime}, \mathrm{SU}(n)}\right)^{-1}+i g U_{\vec{j}, \mathrm{SU}(n)} \mathcal{A}_{\vec{j}, \vec{j}^{\prime}}\left(U_{\vec{j}^{\prime}, \mathrm{SU}(n)}\right)^{-1} \\
& =1+i g \mathcal{A}_{\vec{j}, \vec{j}^{\prime}}^{\prime} \simeq e^{i g \mathcal{A}_{\vec{j}, \vec{j}^{\prime}}^{\prime}} .
\end{aligned}
$$

The total kinetic energy for knots turns into

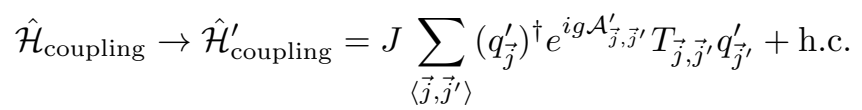

The Hamiltonian does not change,

$$
\hat{\mathcal{H}}_{\text {coupling }}=\hat{\mathcal{H}}_{\text {coupling }}^{\prime} \text {. }
$$

On the other hand, the situation for tempo phase-changing is similar to that for spatial phase-changing. To characterize the tempo twist-writhe locking condition, we introduce a Lagrangian variable $\mathcal{A}_{0, \vec{j}}$ to path integral formulation as

$$
\mathcal{A}_{0, \vec{j}} q_{\vec{j}}^{\dagger} q_{\vec{j}} \text {. }
$$

In continuum limit, we have, $U_{\vec{j}, \mathrm{SU}(n)}(t) \rightarrow U_{\mathrm{SU}(n)}(\vec{x}, t), \mathcal{A}_{\vec{j}, \vec{j}^{\prime}} \rightarrow \mathcal{A}(x)$ and $\mathcal{A}_{0, \vec{j}} \rightarrow \mathcal{A}_{0}(x)$. The non-Abelian gauge symmetry is represented by

$$
q_{\text {quark }}^{\prime} \rightarrow U_{\mathrm{SU}(n)}(\vec{x}, t) q_{\text {quark }}
$$

and

$$
\begin{aligned}
\mathcal{A}_{\mu}(\vec{x}, t) \rightarrow & U_{\mathrm{SU}(n)}(\vec{x}, t) \mathcal{A}_{\mu}(\vec{x}, t)\left(U_{\mathrm{SU}(n)}(\vec{x}, t)\right)^{-1} \\
& +\frac{i}{g}\left(\partial_{\mu} U_{\mathrm{SU}(n)}(\vec{x}, t)\right)\left(U_{\mathrm{SU}(n)}(\vec{x}, t)\right)^{-1}
\end{aligned}
$$


The gauge strength is defined by $\mathcal{G}_{\mu \nu}$ as

$$
\mathcal{G}_{\mu \nu}=\partial_{\mu} \mathcal{A}_{\nu}-\partial_{\nu} \mathcal{A}_{\mu}-i g\left[\mathcal{A}_{\mu}, \mathcal{A}_{\nu}\right]
$$

or

$$
\mathcal{G}_{\mu \nu}=G_{\mu \nu}^{a} \tau^{a}, \quad G_{\mu \nu}^{a}=\partial_{\mu} A_{\nu}^{a}-\partial_{\nu} A_{\mu}^{a}+g f^{a b c} A_{\mu}^{b} A_{\nu}^{c} .
$$

The Lagrangian of Yang-Mills field can only be written as

$$
\mathcal{L}_{\mathrm{YM}}(\mathrm{SU}(n))=-\frac{1}{2} \operatorname{Tr}\left(\mathcal{G}_{\mu \nu} \mathcal{G}^{\mu \nu}\right)+\operatorname{Tr}\left(J_{\mathrm{YM}}^{\mu} \mathcal{A}_{\mu}\right)
$$

where $J_{\mathrm{YM}}^{\mu}=i \bar{q}_{\text {quark }} \gamma^{\mu} q_{\text {quark }}$. The Lagrangian density $\mathcal{L}_{\mathrm{YM}}(\mathrm{SU}(n))$ is invariant under the gauge transformations with an $x$-dependent $U_{\mathrm{SU}(n)}(\vec{x}, t)$.

Finally, we show the physical picture of the $\mathrm{SU}(n)$ gauge field. An extra internal zero also plays the role of source of $\mathrm{SU}(n)$ gauge field and carries color degree of freedom. There are $n$ different colors. For example, for the case of $\mathrm{SU}(3)$, there are three colors, red, blue and green, respectively. The collective modes of SU(3) gauge field are always called gluons that are the fluctuations of internal zeroes. Two colored composite knots interact by exchanging gluons. However, a composite object with $n_{k}=N \cdot n$ internal zeroes ( $N$ is an integer number) are colorless. Because electron is a composite knot without additional internal zeroes $\left(n_{e} \equiv 3\right)$, the fluctuations reorganizing internal zeroes will never affect electrons. So, electrons do not couple the gluons.

\subsection{Examples}

A composite knot-crystal that is projected to coupled zero-lattices naturally gives rise to gauge bosons (such as photons) and fermions (such as electrons). Gauge bosons are vibrations of zero-lattice, while fermions are composite knots of zerolattice. We get the universal effective Lagrangian for a composite knot-crystal as

$$
\mathcal{L}=\mathcal{L}_{\text {fermion }}+\mathcal{L}_{\mathrm{EM}}(\mathrm{U}(1))+\mathcal{L}_{\mathrm{YM}}(\mathrm{SU}(n)) .
$$

The U(1) gauge field characterizes the interaction from the phase fluctuations of internal zero-lattice. The $\mathrm{SU}(n)$ gauge field characterizes the interaction from the fluctuations of twisting number of internal zero-lattice.

\subsubsection{Physics of simple knot-crystal with $n=0$}

For a simple knot-crystal, the knot becomes chargeless electron and the effective model is a free Dirac model of which the effective Lagrangian is

$$
\mathcal{L}=\bar{e}(x) i \gamma^{\mu} \partial_{\mu} e(x)+m_{e} \bar{e}(x) e(x),
$$

where $m_{e}=2 \omega^{*}$.

\subsubsection{Physics of composite knot-crystal with $n=1$}

For the composite knot-crystal without internal winding $(n=0)$, the composite knot becomes electron with $n_{e}=1$. The effectivemodel is $\mathrm{U}(1)$ gauge theory of 
which the effective Lagrangian is given by

$$
\mathcal{L}=\bar{e}(x) i \gamma^{\mu} \partial_{\mu} e(x)+m_{e} \bar{e}(x) e(x)-\frac{1}{4} F_{\mu \nu} F^{\mu \nu}+\mathrm{e} A_{\mu}(x) j_{(e m)}^{\mu}(x),
$$

where $j_{(e m)}^{\mu}(x)=i \bar{e}(x) \gamma^{\mu} e(x) \cdot m_{e}=2 \omega^{*}$ are masses for electron. The phonons $A_{\mu}(x)$ characterize the fluctuations of internal zero-lattice. This model gives QED.

\subsubsection{Physics of composite knot-crystal with $n=2$}

For the composite knot-crystal with $n=2$, the (composite) knots are electron with $n_{e}=2$, quarks with $n_{\text {quark }}=1$. The effective model is $\mathrm{SU}(2) \otimes \mathrm{U}(1)$ gauge theory of which the effective Lagrangian is given by

$$
\begin{aligned}
\mathcal{L}= & \bar{e}(x) i \gamma^{\mu} \partial_{\mu} e(x)+\bar{\psi}_{\text {quark }}(x) i \gamma^{\mu} \partial_{\mu} \psi_{\text {quark }}(x) \\
& +m_{e} \bar{e}(x) e(x)+m_{\text {quark }} \bar{\psi}_{\text {quark }}(x) \psi_{\text {quark }}(x) \\
& -\frac{1}{4} F_{\mu \nu} F^{\mu \nu}+\mathrm{e} A_{\mu}(x) j_{(e m)}^{\mu}(x) \\
& -\frac{1}{2} \operatorname{Tr}\left(\mathcal{G}_{\mu \nu} \mathcal{G}^{\mu \nu}\right)+g \operatorname{Tr}\left(J_{\mathrm{YM}}^{\mu} \mathcal{A}_{\mu}\right),
\end{aligned}
$$

where $g$ is coupling constant of $\mathrm{SU}(2)$ gauge field and $\mathrm{e}=2 e_{0} \cdot m_{\text {quark }}=2 \omega^{*}$ are masses for quark and electron, respectively. The electric current is

$$
j_{(e m)}^{\mu}(x)=i \bar{e}(x) \gamma^{\mu} e(x)+\frac{1}{2} i \bar{\psi}_{\text {quark }}(x) \gamma^{\mu} \psi_{\text {quark }}(x) .
$$

The $\mathrm{SU}(2)$ color current is $J_{\mathrm{YM}}^{a, \mu}=i \bar{\Psi}_{\text {quark }} \gamma^{\mu} T^{a} \Psi_{\text {quark }}$.

The U(1) gauge field characterizes the interaction from the phase fluctuations of internal zero-lattice. The SU(2) gauge field characterizes the interaction from the number of fluctuations of internal zero-lattice.

\section{Emergent Quantum Field Theory for 3-Level Composite Knot-Crystal with $(\mathcal{N}=4, \mathcal{M}=3)$}

In this section, we study the effective theory for knot on a 3-level composite knotcrystal with $(\mathcal{N}=4, \mathcal{M}=3)$. To generate a 3-level composite knot-crystal with $(\mathcal{N}=4, \mathcal{M}=3)$, we symmetrically wind a 2-level double-helix knot-crystal with $(\mathcal{N}=4, \mathcal{M}=2)$ along different spatial directions. As a result, there exists an additional $W$-type zero-lattice by $W$-type projection on the center membrane of A-knot-crystal and B-knot-crystal - the level-3 $W$-type zero-lattice. The effective theory of a 3-level composite knot-crystal with $(\mathcal{N}=4, \mathcal{M}=3)$ reproduces Standard Model, an $\mathrm{SU}_{\text {Strong }}(3) \otimes \mathrm{SU}_{\text {weak }}(2) \otimes \mathrm{U}_{Y}(1)$ gauge theory with Higgs mechanism due to spontaneous symmetry breaking. $\Delta_{\{2,3\}}$ is a very large number, for example, $\Delta_{\{2,3\}} \sim 10^{10}$. We call this composite knot-crystal with $(\mathcal{N}=4, \mathcal{M}=3)$ to be the standard knot-crystal. Figure $6(\mathrm{a})$ is an illustration of the standard knotcrystal. 


\subsection{Classification of composite knots}

In particle physics, there exist different types of fermionic elementary particles: neutrino, electron and quarks. To explain the existence of different types of elementary particles, people try to go beyond SM. Pati and Salam ${ }^{24}$ had proposed preons to be the fundamental constituent particles. Then, other types of models were developed, for example, the Rishon Model proposed simultaneously by Harari and Shupe, ${ }^{25,26}$ the helon model by Bilson-Thompson, ${ }^{27}$ the tangles by Schiller. ${ }^{28}$ In knot physics, we show a correspondence between different types of elementary particles and different types of composite knots.

By trapping different types of zeroes, there exist different types of knots. We use the following number series to label different types of knots:

$$
\left[n_{L_{2}}, n_{L_{1}}\right]
$$

where $n_{L_{2}}$ is the half linking number of level-2 entangled knot-crystals that is equal to the sum of the number of level-2 $T$-type zeroes $n_{L_{2} T}$ and the number of level-3 $W$-type zeroes $n_{L_{3} W}$ as

$$
n_{L_{2}}=n_{L_{2} T}+n_{L_{3} W}
$$

$n_{L_{1}}$ is the half linking number of level-1 entangled vortex-membranes that is equal to the sum of the number of level-1 $T$-type zeroes $n_{L_{1} T}$ and the number of level-2 $W$-type zeroes $n_{L_{2} W}$,

$$
n_{L_{1}}=n_{L_{1} T}+n_{L_{2} W} .
$$

For different types of knots, due to trapping of half linking number of two entangled knot-crystals, we must have $n_{L_{2}}=1$. So, the classification of the knot type is based on the half internal linking number $n_{L_{1}}$ (the half linking number of level-1 entangled vortex-membranes). For a 2-level double-helix knot-crystal with $(\mathcal{N}=4$, $\mathcal{M}=2)$ with an integer hierarchy number $\Delta_{\{1,2\}}=n$, there are $n$ different types of knots with $[1,0],[1,1], \ldots[1, n-1]$. The composite knots with $[1,0]$ correspond to electrons in particle physics and the composite knots with $\left[1, n_{k}\right]\left(n_{k}\right.$ is an integer number, $0<n_{k}<n$ ) correspond to quarks. However, there are two types of knots with $[1,0]$, one is $\left[1_{L_{2} T}, 3\right]$ that is knot with a level-2 $T$-type zero, the other is $\left[1_{L_{3} W}, 0\right]$ that is knot with a level-3 $W$-type zero. The knot with $\left[1_{L_{3} W}, 0\right]$ corresponds to neutrino and the knot with $\left[1_{L_{2} T}, 3\right]$ corresponds to electron.

For example, for the case of $n=3$, the correspondence between four types of knots and elementary particles is given by

A knot with $\left[1_{L_{2} T}, 3\right] \leftrightarrow$ An electron,

A knot with $\left[1_{L_{3} W}, 0\right] \leftrightarrow$ A neutrino,

A knot with $\left[1_{L_{2} T}, 1\right] \leftrightarrow$ A $u$-qurak,

A knot with $\left[1_{L_{2} T}, 2\right] \leftrightarrow \mathrm{A} d$-qurak 
or

$$
\psi_{\left[1_{L_{2} T}, 3\right]} \leftrightarrow e, \quad \psi_{\left[1_{L_{3} W}, 0\right]} \leftrightarrow \nu, \quad \psi_{\left[1_{L_{2} T}, 1\right]} \leftrightarrow u, \quad \psi_{\left[1_{L_{2} T}, 2\right]} \leftrightarrow d .
$$

As a result, in low-energy limit, the effective Lagrangian of knots on a 3-level composite knot-crystal with $(\mathcal{N}=4, \mathcal{M}=3)$ and $\Delta_{\{1,2\}}=n=3$ becomes

$$
\begin{aligned}
\mathcal{L}_{\text {fermion }}(x)= & \bar{\nu} i \gamma^{\mu} \partial_{\mu} \nu+\bar{e} i \gamma^{\mu} \partial_{\mu} e+\bar{d} i \gamma^{\mu} \partial_{\mu} d+\bar{u} i \gamma^{\mu} \partial_{\mu} u+m_{e} \bar{e} e \\
& +m_{d} \bar{d} d+m_{u} \bar{u} u
\end{aligned}
$$

There is no mass term for the left-hand neutrinos.

\subsection{Emergent $\mathrm{SU}_{\mathrm{Strong}}(3) \otimes \mathrm{U}_{\mathrm{EM}}(1)$ gauge theory for the standard knot-crystal}

It was known that a 2-level composite knot-crystal with $(\mathcal{N}=4, \mathcal{M}=2)$ is described by $\mathrm{SU}_{\text {Strong }}(n) \otimes \mathrm{U}_{\mathrm{EM}}(1)$ gauge theory. The $\mathrm{U}(1)$ gauge field characterizes the interaction from the phase fluctuations of internal zero-lattice and the $\mathrm{SU}(n)$ gauge field characterizes the interaction from the fluctuations of twisting number of internal zero-lattice.

For the standard knot-crystal (a 3-level composite knot-crystal with $(\mathcal{N}=4$, $\mathcal{M}=3$ ) and $n=3$ ), we have four types of composite knots: electron with $n_{e}=3$, $d$-quarks with $n_{d \text {-quark }}=1, u$-quarks with $n_{u \text {-quark }}=2$. From the point of view of fluctuations of level-1 internal twistings, without considering the fluctuations of level-3 windings, the effective model is an $\mathrm{SU}_{\mathrm{Strong}}(3) \otimes \mathrm{U}_{\mathrm{EM}}(1)$ gauge theory of which the Lagrangian is

$$
\begin{aligned}
\mathcal{L}= & \bar{\nu} i \gamma^{\mu} \partial_{\mu} \nu+\bar{e} i \gamma^{\mu} \partial_{\mu} e+\bar{d} i \gamma^{\mu} \partial_{\mu} d \\
& +\bar{u} i \gamma^{\mu} \partial_{\mu} u+m_{e} \bar{e} e+m_{d} \bar{d} d+m_{u} \bar{u} u \\
& -\frac{1}{4} F_{\mu \nu} F^{\mu \nu}+\mathrm{e} A_{\mu}(x) j_{(e m)}^{\mu}(x) \\
& -\frac{1}{2} \operatorname{Tr} \mathcal{G}_{\mu \nu} \mathcal{G}^{\mu \nu}+g \operatorname{Tr} J_{\mathrm{YM}}^{\mu} \mathcal{A}_{\mu}
\end{aligned}
$$

where e $=3 e_{0} \cdot m_{\nu}=0, m_{u}=2 \omega^{*}, m_{d}=2 \omega^{*}$, and $m_{e}=2 \omega^{*}$ are masses for neutrino, $u$-quark, $d$-quark and electron, respectively. $g$ is the coupling constant of Yang-Mills gauge field. The electric current is

$$
j_{(e m)}^{\mu}(x)=i \bar{e}(x) \gamma^{\mu} e(x)+i \frac{2}{3} \bar{u}(x) \gamma^{\mu} u(x)+i \frac{1}{3} \bar{d}(x) \gamma^{\mu} d(x) .
$$

The $\mathrm{SU}_{\text {Strong }}(3)$ color current for strong interaction is

$$
J_{\mathrm{YM}}^{a, \mu}=\bar{u}(x) i \gamma^{\mu} T^{a} u(x)+\bar{d}(x) i \gamma^{\mu} T^{a} d(x) .
$$

This model gives QED and QCD. In particular, neutrino does not couple to $\mathrm{SU}_{\text {Strong }}(3) \otimes \mathrm{U}_{\mathrm{EM}}(1)$ gauge fields. 


\subsection{Emergent electroweak $\mathrm{SU}_{\text {weak }}(2) \otimes \mathrm{U}_{Y}(1)$ gauge theory for the standard knot-crystal}

In the above section, from the point of view of fluctuations of level-1 internal twistings, without considering the fluctuations of level-3 windings, the effective model is an $\mathrm{SU}_{\text {Strong }}(3) \otimes \mathrm{U}_{\mathrm{EM}}(1)$ gauge theory. After considering the fluctuations of level-3 windings, the situation changes. In this section, we focus on the effect from the point of view of the fluctuations of level-3 windings and get an electroweak $\mathrm{SU}_{\text {weak }}(2) \otimes \mathrm{U}_{Y}(1)$ gauge field theory. An $\mathrm{SU}_{\text {weak }}(2) \otimes \mathrm{U}_{Y}(1)$ gauge field that couples neutrino (a knot with $\left[1_{L_{3} W}, 0\right]$ ) and electron (a knot with $\left[1_{L_{2} T}, 3\right]$ ) characterizes the interaction by exchanging the fluctuations of different topological number densities in the unit cell of level-3 $W$-type zero-lattice. The fluctuations of $\mathrm{SU}_{\text {weak }}(2)$ gauge theory come from the fluctuations of level-2 writhe number density on a unit cell of level-3 $W$-type zero-lattice and the fluctuations of $\mathrm{U}_{Y}(1)$ gauge theory come from the fluctuations of level-1 twisting number density (the internal zeroes) on a unit cell of level-3 $W$-type zero-lattice.

\subsubsection{Electroweak $\mathrm{SU}_{\text {weak }}(2) \otimes \mathrm{U}_{Y}(1)$ gauge symmetry for leptons}

A neutrino is really half winding with level-3 $W$-type zero and an electron is a halftwisting with internal twisting $n_{e}=\Delta_{(1,2)}=3$. Without considering the charge degree of freedom, we cannot distinguish a uniform distributed left-hand electron from a uniform distributed left-hand neutrino on a unit cell of level-3 zero-lattice. Therefore, we may regard a uniform distributed left-hand neutrino $\nu$ and a uniform distributed left-hand electron $e$ to be the same object of an $\mathrm{SU}_{\text {weak }}(2)$ gauge symmetry on a unit cell of level-3 $W$-type zero-lattice. The fluctuations of internal zeroes on the unit cell of level-3 $W$-type zero-lattice play the role of the Abelian gauge fields for the $\mathrm{U}_{Y}(1)$ gauge symmetry.

We then consider a left-hand electron $e$ to be a composite knot with

$$
n_{e}=\left(n_{e}\right)_{\mathrm{SU}_{\mathrm{weak}}(2)}+\left(n_{e}\right)_{\mathrm{U}_{Y}(1)}=3
$$

where $\left(n_{e}\right)_{\mathrm{SU}_{\text {weak }}(2)}=\frac{3}{2}$ and $\left(n_{e}\right)_{\mathrm{U}_{Y}(1)}=\frac{3}{2}$ and a left-hand neutrino $\nu$ to be a composite knot with

$$
n_{\nu}=\left(n_{\nu}\right)_{\mathrm{SU}_{\mathrm{weak}}(2)}+\left(n_{\nu}\right)_{\mathrm{U}_{Y}(1)}=0
$$

where $\left(n_{\nu}\right)_{\mathrm{SU}_{\text {weak }}(2)}=-\frac{3}{2}$ and $\left(n_{\nu}\right)_{\mathrm{U}_{Y}(1)}=\frac{3}{2}$.

To characterize the property of opposite component of internal zeroes for leptons

$$
\left(n_{e}\right)_{\mathrm{SU}_{\text {weak }}(2)}=-\left(n_{\nu}\right)_{\mathrm{SU}_{\text {weak }}(2)}=\frac{3}{2}
$$

a left-hand neutrino $\nu$ and a left-hand electron $e$ make up a lepton $\mathrm{SU}_{\text {weak }}(2)$ spinor

$$
\psi_{\text {Lepton }}=\left(\begin{array}{l}
\nu \\
e
\end{array}\right) .
$$


The left-handed components of the lepton fields are assigned to doublets of $\mathrm{SU}_{\text {weak }}(2)$ :

$$
\psi_{\text {Lepton }, L}=\frac{1}{2}\left(1+\gamma_{5}\right)\left(\begin{array}{l}
\nu \\
e
\end{array}\right) .
$$

The right-handed components are assigned to singlets of $\mathrm{SU}_{\text {weak }}(2)$ that has no neutrino and we have

$$
\psi_{\text {Lepton, } R}=e_{R}=\frac{1}{2}\left(1-\gamma_{5}\right) e
$$

As a result, we have

$$
\psi_{\text {Lepton }, L} \rightarrow e^{i \boldsymbol{\tau} \boldsymbol{\theta}(\vec{X})} \psi_{\text {Lepton }, L}, \quad \psi_{\text {Lepton }, R} \rightarrow \psi_{\text {Lepton }, R}
$$

with $\tau$ being the three Pauli matrices.

On the other hand, to characterize the property of identical component of internal zeroes for leptons

$$
\left(n_{e}\right)_{\mathrm{U}_{Y}(1)}=\left(n_{\nu}\right)_{\mathrm{U}_{Y}(1)}=\frac{3}{2},
$$

a left-hand neutrino $\nu$ and a left-hand electron $e$ have an additional charge degree of freedom from $\mathrm{U}_{Y}(1)$ gauge symmetry. Such charge degree of freedom is called the supercharge degrees of freedom. The supercharge $\mathcal{Y}$ of left-hand electron and left-hand neutrino with internal zeroes $\left(n_{e}\right)_{\mathrm{U}_{Y}(1)}=\left(n_{\nu}\right)_{\mathrm{U}_{Y}(1)}=\frac{3}{2}$ is

$$
\mathcal{Y}\left(\psi_{\text {Lepton, } L}\right)=-1 \text {. }
$$

An important fact is that unit internal zero has supercharge $Y$ to be $-\frac{2}{3}$, i.e.,

$$
\mathcal{Y}(\text { internal zero })=-\frac{2}{3} .
$$

For a right-hand electron, there are three internal zeroes. The three internal zeroes have supercharge $\mathcal{Y}$ to be $3 \times\left(-\frac{2}{3}\right)=-2$. Therefore, right-hand electrons $e_{R}$ have -2 supercharge as

$$
\mathcal{Y}\left(\psi_{\text {Lepton }, R}\right)=-2
$$

\subsubsection{Electroweak $\mathrm{SU}_{\text {weak }}(2) \otimes \mathrm{U}_{Y}(1)$ gauge symmetry for quarks}

In addition, we consider the electroweak interaction for quarks. In a unit cell of level3 zero-lattice, a $d$-quark has one internal zero $n_{d \text {-quark }}=1$ and an anti- $u$-quark has two internal zero $n_{u^{*} \text {-quark }}=-2$. We may also regard a uniform distributed left-hand $d$-quark and a uniform distributed left-hand anti- $u$-quark to be the same object of an $\mathrm{SU}_{\text {weak }}(2)$ gauge symmetry on the unit cell of level-3 $W$-type zerolattice.

We consider a left-hand $d$-quark to be a composite knot as

$$
n_{d-\text { quark }}=\left(n_{d-\text { quark }}\right)_{\mathrm{SU}_{\text {weak }}(2)}+\left(n_{d-\text { quark }}\right)_{\mathrm{U}_{Y}(1)},
$$


where $\left(n_{d-\text { quark }}\right)_{\mathrm{SU}_{\text {weak }}(2)}=\frac{3}{2}$ and $\left(n_{d-\text { quark }}\right)_{\mathrm{U}_{Y}(1)}=-\frac{1}{2}$ and a left-hand $u$-quark to be a knot with 1.5 internal zero,

$$
n_{u^{*}-\text { quark }}=\left(n_{u^{*}-\text { quark }}\right)_{\mathrm{SU}_{\text {weak }}(2)}+\left(n_{\mathrm{u}^{*}-\text { quark }}\right)_{\mathrm{U}_{Y}(1)},
$$

where $\left(n_{u-\text { quark }}\right)_{\mathrm{SU}_{\text {weak }}(2)}=-\frac{3}{2}$ and $\left(n_{u-\text { quark }}\right)_{\mathrm{U}_{Y}(1)}=-\frac{1}{2}$.

To characterize the property of opposite component of internal zeroes for $\bar{u}$ and $d$ as

$$
\left(n_{d-\text { quark }}\right)_{\mathrm{SU}_{\text {weak }}(2)}=-\left(n_{u-\text { quark }}\right)_{\mathrm{SU}_{\text {weak }}(2)}=\frac{3}{2}
$$

$\bar{u}$ and $d$ make up a quark $\mathrm{SU}_{\text {weak }}(2)$ spinor $^{29}$

$$
\psi_{\text {quark }}=\left(\begin{array}{l}
\bar{u} \\
d
\end{array}\right) \text {. }
$$

They are fractionally charged and come each in three "colors",

$$
\psi_{\text {quark }, L}=\frac{1}{2}\left(1+\gamma_{5}\right)\left(\begin{array}{l}
\bar{u} \\
d
\end{array}\right) .
$$

On the other hand, to characterize the property of identical component of internal zeroes for $\bar{u}$ and $d$ as

$$
\left(n_{d-\text { quark }}\right)_{\mathrm{SU}_{\text {weak }}(2)}=\left(n_{u-\text { quark }}\right)_{\mathrm{SU}_{\text {weak }}(2)}=-\frac{1}{2},
$$

a left-hand $d$-quark and a left-hand anti- $u$-quark have the supercharge $\mathcal{Y}$ to be

$$
\mathcal{Y}\left(\psi_{\text {quark }, L}\right)=-\frac{1}{2} \times\left(-\frac{2}{3}\right)=\frac{1}{3} \text {. }
$$

Since a unit internal zero has supercharge $\mathcal{Y}$ to be $-\frac{2}{3}$, we find that the supercharge for quarks $u_{R}, d_{R}$ to be

$$
\mathcal{Y}\left(u_{R}\right)=-\frac{4}{3}, \quad \mathcal{Y}\left(d_{R}\right)=-\frac{2}{3} .
$$

\subsubsection{Effective Lagrangian of electroweak $\mathrm{SU}_{\text {weak }}(2) \otimes \mathrm{U}_{Y}(1)$ gauge theory}

Finally, we write down the effective Lagrangian of electroweak $\mathrm{SU}_{\text {weak }}(2) \otimes \mathrm{U}_{Y}(1)$ gauge theory:

$$
\begin{aligned}
\mathcal{L}= & \mathcal{L}_{\text {fermion }}+\mathcal{L}_{Y}\left(\mathrm{U}_{Y}(1)\right)+\mathcal{L}_{\text {weak }}\left(\mathrm{SU}_{\text {weak }}(2)\right) \\
= & \operatorname{Tr}\left[\bar{\psi}_{L} i \gamma^{\mu}\left(\partial_{\mu}-i g_{\text {weak }} W_{\mu}+i \frac{g^{\prime}}{2} B_{\mu}\right) \psi_{L}\right] \\
& +\bar{\psi}_{R} i \gamma^{\mu}\left(\partial_{\mu}+i g^{\prime} B_{\mu}\right) \psi_{R}-\frac{1}{4} B_{\mu \nu} B^{\mu \nu}-\operatorname{Tr}\left(\frac{1}{2} W_{\mu \nu} W^{\mu \nu}\right),
\end{aligned}
$$

where $W_{\mu}$ and $B_{\mu}$ denote the gauge fields associated to weak $\mathrm{SU}_{\text {weak }}(2)$ and supercharge $\mathrm{U}_{Y}(1)$, respectively, of which the corresponding field strengths are $W_{\mu \nu}$ and 
$B_{\mu \nu}$. The two coupling constants $g_{\text {weak }}$ and $g^{\prime}$ correspond to the groups $\mathrm{SU}_{\text {weak }}(2)$ and $\mathrm{U}_{Y}(1)$, respectively. Because neutrino has only left-hand degrees of freedom, the charged $W$ 's couple only to the left-handed components of the lepton fields.

Electroweak $\mathrm{SU}_{\text {weak }}(2) \otimes \mathrm{U}_{Y}(1)$ gauge symmetry comes from the redundancies of 2-level composite knots and those of 1-level internal knots in unit cell of level-3 zero-lattices. As a result, the fluctuations of $\mathrm{SU}_{\text {weak }}(2)$ gauge theory come from the fluctuations of writhe number density on a unit cell of level-3 $W$-type zerolattice and the fluctuations of $\mathrm{U}_{Y}(1)$ gauge theory come from the residue phase fluctuations of internal twisting on a unit cell of level-3 zero-lattice.

\subsubsection{Higgs mechanism and spontaneous symmetry breaking for standard knot-crystal}

We then study the properties of angular velocity of leapfrogging motion $\omega(\vec{X}, t)$ (that is really the Higgs field $\Phi(\vec{X}, t) / 2$ in Standard Model), that is

$$
\psi^{\prime}(\vec{X}, t)=e^{2 i \tau_{x} \omega(\vec{X}, t) \cdot t} \cdot \psi(\vec{X}, t) .
$$

Consequently, the effect of leapfrogging motion is to change $\psi_{L}(\vec{X})$ to $\psi_{R}(\vec{X})$ and there appears an extra term in Hamiltonian as $\psi^{\dagger} \omega \tau_{x} \psi=\psi_{R}^{\dagger} \omega \psi_{L}$. Because of

$$
\mathcal{Y}\left(\psi_{\text {Lepton }, L}\right)=-1, \quad \mathcal{Y}\left(\psi_{\text {Lepton }, R}\right)=0,
$$

the $\mathrm{U}_{Y}(1)$ supercharge of $\omega(\vec{X}, t)$ must be

$$
\mathcal{Y}(\omega(\vec{X}, t))=\mathcal{Y}\left(\psi_{\text {Lepton }, R}\right)-\mathcal{Y}\left(\psi_{\text {Lepton }, L}\right)=1 \text {. }
$$

On the other hand, due to

$$
\psi_{L}(\vec{X}) \rightarrow e_{L}^{i \boldsymbol{\tau} \boldsymbol{\theta}(\vec{X})} \psi(\vec{X}), \quad \psi_{R}(\vec{X}) \rightarrow \psi_{R}(\vec{X})
$$

$\omega(\vec{X}, t)$ must be an $\mathrm{SU}_{\text {weak }}(2)$ complex doublet as

$$
\omega(\vec{X}, t)=\left(\begin{array}{c}
\phi^{+} \\
\phi^{0}
\end{array}\right), \quad \omega(\vec{X}, t) \rightarrow e^{i \tau \boldsymbol{\theta}(X)} \omega(\vec{X}, t) .
$$

As a result, the fluctuating leapfrogging angular velocity of a standard knotcrystal $\omega_{0} \rightarrow \omega(\vec{X}, t)$ plays the role of Higgs field $\Phi(\vec{X}, t)$ in Standard Model..$^{21}$

Next, we write down the Lagrangian of the leapfrogging field $\omega(\vec{X}, t)$. Because the leapfrogging field $\omega(\vec{X}, t)$ is an $\mathrm{SU}_{\text {weak }}(2)$ complex doublet and has supercharge $\mathcal{Y}=1$, we get the kinetic term of leapfrogging field $\omega(\vec{X}, t)$ as

$$
\left|\left(\partial_{\mu}-i g_{\text {weak }} \frac{\tau}{2} \cdot \vec{W}_{\mu}-i \frac{g^{\prime}}{2} B_{\mu}\right) \omega(\vec{X}, t)\right|^{2} .
$$

To obtain the finite leapfrogging velocity, we also add a phenomenological term $V(\omega(\vec{X}, t))$.

Finally, by adding Yukawa coupling between the Higgs field and fermions, the full Lagrangianof $\omega(\vec{X}, t)$ is given by 


$$
\begin{aligned}
\mathcal{L}_{\text {Higgs }}= & \left|\left(\partial_{\mu}-i g_{\text {weak }} \frac{\boldsymbol{\tau}}{2} \cdot \vec{W}_{\mu}-i \frac{g^{\prime}}{2} B_{\mu}\right) \omega(\vec{X}, t)\right|^{2} \\
& -V(\omega(\vec{X}, t))+\bar{\psi}_{\text {Lepton }, L} G_{\text {Lepton }} \omega(\vec{X}, t) \bar{\psi}_{\text {Lepton }, R} \\
& +\bar{\psi}_{\text {quark }, L} G_{\text {quark }} \omega(\vec{X}, t) \psi_{\text {quark }, R}+\text { h.c. },
\end{aligned}
$$

where $G_{\text {Lepton }}=G_{\text {quark }}=2$.

A finite leapfrogging angular velocity is given by minimizing $\omega(\vec{X}, t)$ of which the expected value is $\omega^{*}$. Then, the weak gauge symmetry is spontaneously broken, we get a finite angular velocity of leapfrogging motion as

$$
\langle\omega(\vec{X}, t)\rangle=\frac{1}{\sqrt{2}}\left(\begin{array}{c}
0 \\
\omega^{*}
\end{array}\right)+\delta \omega(\vec{X}, t) .
$$

A finite angular velocity of leapfrogging motion creates a mass term for the electrons $m_{e}=\frac{1}{\sqrt{2}} G_{e} \omega^{*}$. For the system with finite angular velocity of leapfrogging motion, we produce masses for the quarks given by $m_{u}=G_{u} \omega^{*}, m_{d}=G_{d} \omega^{*}$. Because there is no right-hand neutrino, the mass of neutrino is zero, $m_{\nu} \equiv 0$. In addition, the Higgs field also has mass that is $m_{\text {Higgs }} \neq 0$.

The finite angular velocity of leapfrogging motion plays the role of Higgs condensation and the Higgs mechanism of 3-level composite knot-crystal with $(\mathcal{N}=4$, $\mathcal{M}=3$ ) breaks the original gauge symmetry according to $\mathrm{SU}_{\text {weak }}(2) \otimes \mathrm{U}_{Y}(1) \rightarrow$ $\mathrm{U}_{\mathrm{EM}}(1)$.

As a result, the $\mathrm{SU}_{\text {weak }}(2)$ gauge fields obtain masses from the following terms ${ }^{22}$ :

$$
\frac{1}{8}\left(\omega^{*}\right)^{2}\left[\left(g_{\text {weak }}\right)^{2}\left(W_{\mu}^{1} W^{1 \mu}+W_{\mu}^{2} W^{2 \mu}\right)+\left(g^{\prime} B_{\mu}-g_{\text {weak }} W_{\mu}^{3}\right)^{2}\right] .
$$

The mass for the charged vector bosons $W_{\mu}^{ \pm}=\left(W_{\mu}^{1} \mp i W_{\mu}^{2}\right) / \sqrt{2}$ is

$$
m_{W}=\frac{\omega^{*} g_{\text {weak }}}{2} .
$$

After diagonalization, the gauge fields $B_{\mu}$ and $W_{\mu}^{3}$ are transformed into gauge fields $Z_{\mu}$ and $A_{\mu}$ from the following relations:

$$
Z_{\mu}=W_{\mu}^{3} \cos \theta_{W}-B_{\mu} \sin \theta_{W}, \quad A_{\mu}=B_{\mu} \cos \theta_{W}+W_{\mu}^{3} \sin \theta_{W}
$$

with $\tan \theta_{W}=g^{\prime} / g_{\text {weak }}$, of which the masses are

$$
m_{Z}=\frac{\omega^{*}\left(\left(g_{\text {weak }}\right)^{2}+g^{\prime 2}\right)^{1} / 2}{2}=\frac{m_{W}}{\cos \theta_{W}}, \quad m_{A}=0 .
$$

The "Weinberg angle" $\theta_{W}$ becomes the angle between the original $\mathrm{U}(1)$ and the one left unbroken.

The neutral gauge bosons $A_{\mu}$ are massless and will be identified with the photons. Now, the gauge symmetry $\mathrm{U}_{\mathrm{EM}}(1)$ accompanying $A_{\mu}$ is to change the position of the internal zeroes of the composite knots that will never be broken. That is

$$
\psi(\vec{X})=\left(\begin{array}{l}
\psi_{L}(\vec{X}) \\
\psi_{R}(\vec{X})
\end{array}\right) \rightarrow\left(\begin{array}{l}
\psi_{L}(x) e^{-i \mathrm{e} \phi(\vec{X})} \\
\psi_{R}(x) e^{-i \mathrm{e} \phi(\vec{X})}
\end{array}\right) \rightarrow e^{-i \mathrm{e} \phi(\vec{X})} \psi(\vec{X}) .
$$


Then, the electric charge e will be a linear combination of $T_{3}$ and $\mathcal{Y}$ as

$$
\mathrm{e}=T_{3}+\frac{\mathcal{Y}}{2}
$$

\subsection{Unified theory of standard knot-crystal}

Finally, we derive the unified theory of standard knot-crystal by considering all points of view. The low-energy effective theory is just the Standard Model - an $\mathrm{SU}_{\text {Strong }}(3) \otimes \mathrm{SU}_{\text {weak }}(2) \otimes \mathrm{U}_{Y}(1)$ gauge theory with Higgs mechanism due to spontaneous symmetry breaking.

Before considering Higgs condensation or $\omega^{*}=0$, the low-energy effective Lagrangian density is

$$
\mathcal{L}_{\mathrm{SM}}=\mathcal{L}_{\text {fermion }}+\mathcal{L}_{Y}\left(\mathrm{U}_{Y}(1)\right)+\mathcal{L}_{\text {strong }}\left(\mathrm{SU}_{\text {Strong }}(3)\right)+\mathcal{L}_{\text {weak }}\left(\mathrm{SU}_{\text {weak }}(2)\right)+\mathcal{L}_{\text {Higgs }},
$$

where

$$
\begin{aligned}
& \mathcal{L}_{\text {fermion }}= \operatorname{Tr}\left[\bar{\psi}_{L} i \gamma^{\mu}\left(\partial_{\mu}-i g_{\text {weak }} \frac{\boldsymbol{\tau}}{2} \cdot \vec{W}_{\mu}+i \frac{g^{\prime}}{2} B_{\mu}\right) \psi_{L}\right]+\bar{\psi}_{R} i \gamma^{\mu}\left(\partial_{\mu}+i g^{\prime} B_{\mu}\right) \psi_{R} \\
&+\operatorname{Tr}\left[\bar{\psi}_{\text {quark }, L} i\left(\partial_{\mu}-i g_{\text {weak }} \frac{\tau}{2} \cdot \vec{W}_{\mu}-i \frac{g^{\prime}}{6} B_{\mu}\right) \psi_{\text {quark }, L}\right. \\
&\left.+\bar{u}_{R} i\left(\partial_{\mu}+i \frac{2 g^{\prime}}{3} B_{\mu}\right) u_{R}+\bar{d}_{R} i\left(\partial_{\mu}+i \frac{g^{\prime}}{3} B_{\mu}\right) d_{R}\right] \\
&+\mathrm{e} A_{\mu}(x) j_{(e m)}^{\mu}+g \operatorname{Tr}\left(J_{\mathrm{YM}}^{\mu} \mathcal{A}_{\mu}\right), \\
& \mathcal{L}_{Y}\left(\mathrm{U}_{Y}(1)\right)=-\frac{1}{4} B_{\mu \nu} B^{\mu \nu}, \\
& \mathcal{L}_{\text {strong }}\left(\mathrm{SU}_{\text {Strong }}(3)\right)=-\frac{1}{2} \operatorname{Tr}\left(\mathcal{G}_{\mu \nu} \mathcal{G}^{\mu \nu}\right), \\
& \mathcal{L}_{\text {weak }}\left(\mathrm{SU}_{\text {weak }}(2)\right)=-\operatorname{Tr}\left(\frac{1}{2} W_{\mu \nu} W^{\mu \nu}\right), \\
& \mathcal{L}_{\text {Higgs }}=\left|\left(\partial_{\mu}-i g_{\text {weak }} \frac{\tau}{2} \cdot \vec{W}_{\mu}-i \frac{g^{\prime}}{2} B_{\mu}\right) \omega\right|^{2}-V(\omega)+\bar{\psi}_{\text {quark }, L} G_{\text {quark }} \omega \psi_{\text {quark }, R} \\
&+ \bar{\psi}_{\text {Lepton }, L} G_{\text {Lepton }} \omega \psi_{\text {Lepton }, R}+\text { h.c. }
\end{aligned}
$$

The electric current is

$$
j_{(e m)}^{\mu}=i \bar{e} \gamma^{\mu} e+i \frac{2}{3} \bar{u} \gamma^{\mu} u+i \frac{1}{3} \bar{d} \gamma^{\mu} d .
$$

The color current is

$$
J_{\mathrm{YM}}^{a, \mu}=\bar{u} i \gamma^{\mu} T^{a} u+\bar{d} i \gamma^{\mu} T^{a} d
$$


After considering the Higgs condensation $\omega^{*} \neq 0$, we have the low-energy effective Lagrangian as

$$
\begin{aligned}
\mathcal{L}_{\mathrm{SM}}= & \mathcal{L}_{\text {fermion }}+\mathcal{L}\left(\mathrm{U}_{\mathrm{EM}}(1)\right)+\mathcal{L}_{\text {weak }}\left(\mathrm{SU}_{\text {weak }}(2)\right)+\mathcal{L}_{\text {strong }}\left(\mathrm{SU}_{\text {Strong }}(3)\right)+\mathcal{L}_{\text {Higgs }} \\
= & \bar{\nu} i \gamma^{\mu} \partial_{\mu} \nu+\bar{e}_{R} i \gamma^{\mu} \partial_{\mu} e \\
& +\bar{u} i \gamma^{\mu} \partial_{\mu} u+\bar{d} i \gamma^{\mu} \partial_{\mu} d+m_{e} \bar{e} e+m_{d} \bar{d} d+m_{u} \bar{u} u . \\
& -\frac{1}{4} F_{\mu \nu} F^{\mu \nu}-\operatorname{Tr}\left(\frac{1}{2} W_{\mu \nu} W^{\mu \nu}\right)-\frac{1}{2} \operatorname{Tr}\left(\mathcal{G}_{\mu \nu} \mathcal{G}^{\mu \nu}\right) \\
& +\mathrm{e} A_{\mu}(x) j_{(e m)}^{\mu}+g \operatorname{Tr}\left(J_{\mathrm{YM}}^{\mu} \mathcal{A}_{\mu}\right) \\
& +\frac{1}{8}\left(\omega^{*}\right)^{2}\left[\left(g_{\text {weak }}\right)^{2}\left(W_{\mu}^{1} W^{1 \mu}+W_{\mu}^{2} W^{2 \mu}\right)+\left(g^{\prime} B_{\mu}-g_{\text {weak }} W_{\mu}^{3}\right)^{2}\right] \\
& +g_{\text {weak }} \operatorname{Tr}\left(W_{\mu} j_{w}^{\mu}\right)+\left|\partial_{\mu} \omega\right|^{2}+m_{\text {Higgs }}|\omega|^{2}+\cdots,
\end{aligned}
$$

where $j_{w}^{\mu}$ is the weak current. This is exact one-flavor Standard Model, an $\mathrm{SU}_{\text {Strong }}(3) \otimes(\mathrm{SU}(2))_{\text {weak }} \otimes \mathrm{U}_{Y}(1)$ gauge theory with Higgs mechanism due to spontaneous symmetry breaking.

\section{Summary and Discussion}

In the end, we give a summary. In this paper, knot dynamics on composite knot-crystal is studied. From knot physics, the knot-crystal becomes fundamental physical object of which elementary excitations are gauge fields and fermionic particles (knots). In particular, for a special composite knot-crystal - standard knot-crystal, the low-energy effective theory is just the Standard Model - an $\mathrm{SU}_{\text {Strong }}(3) \otimes \mathrm{SU}_{\text {weak }}(2) \otimes \mathrm{U}_{Y}(1)$ gauge theory with Higgs mechanism due to spontaneous symmetry breaking. The knot physics provides a way to unify all gauge fields and elementary fermionic excitations. In Table 1, we give the correspondence between different quantum field theories in modern physics and the different composite knot-crystals in knot physics.

Grand Unified Theory (GUT) is a dream of physicists to unify all nongravitational interactions. There are several approaches towards GUT. String theory is a possible theory of GUT. $\frac{32}{2}$ According to string theory, matter consists of vibrating

Table 1. The correspondence between different quantum field theories in modern physics and the different composite knot-crystals in knot physics.

\begin{tabular}{cl}
\hline Composite knot-crystal & \multicolumn{1}{c}{ Quantum field theory } \\
\hline $\mathcal{N}=1$ and $\mathcal{M}=1$ & Weyl Fermion model \\
$\mathcal{N}=2$ and $\mathcal{M}=1$ & Dirac Fermion model \\
$\mathcal{N}=2$ and $\mathcal{M}=2$ & Weak $\mathrm{SU}(2)$ gauge field theory \\
$\mathcal{N}=4$ and $\mathcal{M}=2$ & $\mathrm{SU}(n)^{*} \mathrm{U}(1)$ gauge field theory \\
$\mathcal{N}=4$ and $\mathcal{M}=3$ & Standard Model \\
\hline
\end{tabular}


strings (or strands) and different oscillatory patterns of strings become different particles with different masses. In condensed matter physics, the idea of our universe as an "emergent" phenomenon has become increasingly popular. In emergence approach, a deeper and unified understanding of the universe is developed based on a complicated many-body system. Different quantum fields correspond to different many-body systems: the vacuum corresponds to the ground state and the elementary particles correspond to the excitations of the systems. According to string-net picture proposed by Wen, our universe (such as gauge interaction, Fermi statistics, etc.) emerges from a frustrated quantum spin model. $\frac{33}{}$ In addition, there exist many other proposals of GUT from different points of view, such as loop quantum gravity theory, ${ }^{34,35}$ Lisi's E8 theory, ${ }^{36}$ Schiller's Strand Model, 28 etc.

Based on knot physics, we may guess that our universe becomes a composite knot-crystal - standard knot-crystal, of which the hierarchy series is $\{3, N\}$, where $N$ is a very large number, $N \gg 1$.

In this paper, there are two important issues that we do not discuss: (1) the flavor physics, including the origin of three-flavor, the value of each elements of Cabibbo-Kobayashi-Maskawa mass matrix ${ }^{37}$ and the mechanism of weak chargeparity (CP) violence; (2) quark confinement that may be relevant to the dynamics of internal knots inside the quarks. In the future, we will study these issues and develop a complete knot theory for particle physics and quantum field theory.

\section{Acknowledgment}

This work is supported by NSFC Grant No. 11674026.

\section{References}

1. W. Thomson, Philos. Mag. 10, 155 (1880).

2. R. J. Donnelly, Quantized Vortices in Helium II (Cambridge University Press, Cambridge, 1991).

3. B. V. Svistunov, Phys. Rev. B 52, 3647 (1995).

4. W. F. Vinen, Phys. Rev. B 61, 1410 (2000).

5. F. W. Dyson, Philos. Trans. R. Soc. Lond. A 184, 1041 (1893).

6. W. M. Hicks, Proc. R. Soc. Lond. A 102, 111 (1922).

7. A. V. Borisov, A. A. Kilin and I. S. Mamaev, Regul. Chaotic Dyn. 18, 33 (2013).

8. D. H. Wacks, A. W. Baggaley and C. F. Barenghi, Phys. Fluids 26, 027102 (2014).

9. R. M. Caplan et al., Phys. Fluids 26, 097101 (2014).

10. N. Hietala et al., arXiv:1603.06403.

11. D. Kleckner and W. T. M. Irvine, Nat. Phys. 9, 253 (2013).

12. D. S. Hall et al., Nat. Phys. 12, 478 (2016).

13. S. P. Kou, Int. J. Mod. Phys. B 31, 1750241 (2017).

14. B. Khesin, arXiv:1201.5914.

15. H. K. Moffatt and R. L. Ricca, Proc. R. Soc. Lond. A 439, 411 (1992).

16. K. Klenin and J. Langowski, Biopolymers 54, 307 (2000).

17. S. P. Kou, arXiv:1706.06879.

18. H. Weyl, Z. Phys. 56, 330 (1929).

19. C. N. Yang and R. L. Mills, Phys. Rev. 96, 191 (1954). 
20. T. D. Lee and C. N. Yang, Phys. Rev. 104, 254 (1956).

21. P. W. Higgs, Phys. Lett. 12, 132 (1964).

22. S. Weinberg, Phys. Rev. Lett. 19, 1264 (1967).

23. T. Kaluza, Sitzungsberichte Pruss. Acad. Sci. 966 (1921); O. Klein, Zeitschrift für Physik A 37, 895 (1926); O. Klein, Nature 118, 516 (1926).

24. J. C. Pati and A. Salam, Phys. Rev. D 10, 275 (1974).

25. H. Harari, Phys. Lett. B 86, 83 (1979).

26. M. Shupe, Phys. Lett. B 86, 87 (1979).

27. S. Bilson-Thompson, arXiv:hep-ph/0503213.

28. C. Schiller, A Fascinating Speculation: The Strand Model.

29. S. L. Glashow, J. Iliopoulos and L. Maiani, Phys. Rev. D 2, 1285 (1970).

30. C. Quigg, Gauge Theories of the Strong, Weak, and Electromagnetc Interactions (Addison-Wesley, Menlo Park, 1983).

31. H. Georgi and S. Glashow, Phys. Rev. Lett. 32, 438 (1974).

32. M. Kaku, Introduction to Superstring and $M$-Theory, 2nd edn. (Springer-Verlag, New York, 1999).

33. X.-G. Wen, Quantum Field Theory of Many-Body Systems (Oxford University Press, Oxford, 2004).

34. A. Ashtekar, Phys. Rev. Lett. 57, 2244 (1986); C. Rovelli, arXiv:1102.3660; C. Rovelli (Cambridge University Press, Cambridge, 2004).

35. S. O. Bilson-Thompson, F. Markopoulou and L. Smolin, Class. Quantum Grav. 24, 3975 (2007).

36. A. G. Lisi, arXiv:0711.0770 [hep-th].

37. N. Cabibbo, Phys. Rev. Lett. 10, 531 (1963); M. Kobayashi and T. Maskawa, Prog. Theor. Phys. 49, 652 (1973). 